\title{
Resolubilidade e irresolubilidade de espaços topológicos
}

\author{
Ana Carolina Boero \\ DISSERTAÇÃO APRESENTADA \\ $\mathrm{AO}$ \\ INSTITUTO DE MATEMÁTICA E ESTATÍSTICA \\ DA \\ UNIVERSIDADE DE SÃO PAULO \\ PARA \\ OBTENÇÃO DO TÍTULO DE MESTRE \\ $\mathrm{EM}$ \\ CIÊNCIAS \\ Área de Concentração: Matemática \\ Orientador: Prof. Dr. Antonio de Padua Franco Filho
}

Durante a elaboração deste trabalho, a autora recebeu apoio financeiro do CNPq.

- São Paulo, 09 de março de 2007 - 


\title{
Resolubilidade e irresolubilidade de espaços topológicos
}

\author{
Este exemplar corresponde à redação \\ final da dissertação devidamente corrigida \\ e defendida por Ana Carolina Boero e \\ aprovada pela comissão julgadora.
}

São Paulo, 09 de março de 2007.

\section{Banca examinadora:}

Prof. Dr. Antonio de Padua Franco Filho (orientador)

IME-USP

Profa. Dra. Ofelia Teresa Alas

IME-USP

Prof. Dr. Samuel Gomes da Silva

UFBA 
Dedico esta dissertação a você, $Z$.

Porque existe um único amor neste mundo. 


\section{Agradecimentos}

Agradeço ao meu orientador, Antonio de Padua Franco Filho, pelo incentivo constante, por sua paciência com minhas faltas e pelos valiosos ensinamentos que me dispensou.

Agradeço aos professores Ofelia Teresa Alas e Samuel Gomes da Silva por terem aceitado

participar da comissão julgadora desta dissertação de mestrado. É, para mim, uma imensa honra tê-los como membros da banca examinadora e espero poder retribuir à altura, com um bom trabalho.

Agradeço o inestimável apoio de meus professores, os quais transmitiram seu conhecimento com dedicação e profissionalismo.

Agradeço aos funcionários do Instituto de Matemática e Estatística da Universidade de São Paulo, pela seriedade com que cumprem seus deveres e pela disposição exemplar.

Agradeço aos meus amigos pelas angústias divididas e pelas alegrias multiplicadas.

Agradeço aos meus pais, ao meu irmão e à minha avó que, em diversas ocasiões, renunciaram seus sonhos para que eu pudesse realizar os meus.

Agradeço ao meu namorado, José Antonio Verderesi, por ser o alicerce do meu encanto pela vida. Além de suas inúmeras qualidade pessoais, seu amor pela Matemática, sua grande erudição e suas brilhantes idéias tornaram a realização deste trabalho extremamente prazerosa.

Agradeço, por fim, ao CNPq pelo apoio financeiro. 


\section{Resumo}

O principal objetivo deste trabalho é apresentar um estudo sistemático da teoria dos espaços topológicos resolúveis e irresolúveis. Enfocaremos diversas propriedades inerentes aos mesmos, incluindo uma exposição meticulosa de técnicas utilizadas na construção de espaços topológicos irresolúveis e sem pontos isolados. Dado um cardinal $\kappa>1$, exibiremos exemplos de espaços topológicos que são $\kappa$-resolúveis, mas que não são $\kappa^{+}$-resolúveis. Mostraremos, ainda, que se um espaço topológico for $n$-resolúvel, para todo número natural $n>1$, o mesmo será $\omega$-resolúvel. Provaremos, contudo, que se $\lambda$ é um cardinal tal que $\omega<\operatorname{cf}(\lambda)=\lambda$, existe um espaço topológico que é $\mu$-resolúvel, para todo cardinal $\mu<\lambda$, mas que não é $\lambda$-resolúvel. O cerne desta dissertação refere-se à construção, em ZFC, de um subespaço enumerável, denso e submaximal de $2^{\mathfrak{c}}$. Com o auxílio de algumas idéias introduzidas em [12], elaboramos uma nova demonstração deste resultado, feita diretamente no cubo de Cantor em questão. 


\section{Abstract}

The main purpose of this work is to study the theory of resolvable and irresolvable topological spaces. We shall introduce many properties of these spaces and we shall give special attention to some techniques used in the construction of irresolvable topological spaces without isolated points. Given a cardinal $\kappa>1$, we will present some examples of topological spaces which are $\kappa$-resolvable, but not $\kappa^{+}$-resolvable. Besides, we will show that if a topological space is $n$-resolvable, for every natural number $n>1$, then it is $\omega$ resolvable too. Nevertheless, we shall prove that if $\lambda$ is a cardinal with $\omega<\operatorname{cf}(\lambda)=\lambda$, there is a topological space which is $\mu$-resolvable, for each cardinal $\mu<\lambda$, but that is not $\lambda$-resolvable. The backbone of this dissertation is the construction, in ZFC, of a countable,

dense and submaximal subspace of $2^{\mathfrak{c}}$. We created a new proof of this result, based in some ideas presented in [12]. 


\section{Sumário}

$\begin{array}{lr}\text { Introdução } & 1\end{array}$

1 Definições e resultados preliminares 4

1.1 Teoria $\operatorname{dos}$ Conjuntos . . . . . . . . . . . . . . . . . . 5

1.2 Topologia Geral . . . . . . . . . . . . . . . . . . . . . . . . 9

2 Espaços topológicos resolúveis $\quad 15$

2.1 A noção de resolubilidade . . . . . . . . . . . . . . . . . . . . 16

2.2 A noção de $\kappa$-resolubilidade . . . . . . . . . . . . . . . . . . . . . . . . . . . 29

3 Espaços topológicos irresolúveis $\quad 41$

3.1 Graus de irresolubilidade . . . . . . . . . . . . . . . . . . . . . . 42

3.2 Expansões de espaços topológicos . . . . . . . . . . . . . . . . . 54

4 Resolubilidade finita e $\omega$-resolubilidade $\quad 69$

5 Irresolubilidade no cubo de Cantor $2^{\mathfrak{c}} \quad 80$

5.1 Um subespaço enumerável, denso e submaximal de $2^{\mathfrak{c}} \ldots \ldots$. . . . . . 81

5.2 A não existência de um subespaço denso e maximal de $2^{\mathfrak{c}} \ldots \ldots$. . . . . . 97 
6 Espaços $\mathcal{D}$-forçados $\quad 101$

6.1 Introdução aos espaços $\mathcal{D}$-forçados . . . . . . . . . . . . . . . . . . . . 102

6.2 Famílias independentes de partições . . . . . . . . . . . . . . . . 112

6.3 Aplicações à resolubilidade . . . . . . . . . . . . . . . . . . . . . . 123

Referências Bibliográficas

Índice Remissivo 


\section{Introdução}

Os conceitos de resolubilidade e irresolubilidade de espaços topológicos foram introduzidos por E. Hewitt, em 1943. Desde então, diversas noções relacionadas aos mesmos têm atraído o interesse de topólogos e estudiosos da área.

A finalidade deste trabalho é apresentar um estudo sistemático da teoria dos espaços topológicos resolúveis e irresolúveis. São seis os capítulos que compõem esta dissertação.

O primeiro deles tem por objetivo estabelecer a notação que será utilizada ao longo do texto, além de destacar resultados preliminares e definições essenciais à compreensão do leitor.

O segundo capítulo será dedicado aos espaços topológicos resolúveis e suas principais propriedades. Dizemos que um espaço topológico é resolúvel se o mesmo possui dois subconjuntos densos complementares. Caso contrário, o espaço em questão é dito irresolúvel. Mostraremos, na primeira seção deste capítulo, que todo espaço métrico sem pontos isolados é resolúvel, bem como todo espaço topológico localmente compacto, $T_{2}$ e denso em si mesmo. Provaremos, ainda, que todo espaço topológico $T_{0}$, sem pontos isolados e que satisfaz o segundo axioma de enumerabilidade é resolúvel. Generalizaremos, na seção posterior, a noção de resolubilidade de um espaço topológico. Dado um cardinal $\kappa>1$, dizemos que um espaço topológico é $\kappa$-resolúvel se o mesmo possui $\kappa$ subconjuntos densos, dois a dois disjuntos. Introduziremos, também, o conceito de espaço topológico 
maximalmente resolúvel e demonstraremos que todo espaço topológico $T_{0}$, denso em si mesmo e que satisfaz o segundo axioma de enumerabilidade é maximalmente resolúvel. Por fim, provaremos que todo espaço topológico metrizável e sem pontos isolados é maximalmente resolúvel, bem como todo topológico localmente compacto, $T_{2}$ e denso em si mesmo.

Embora os resultados apresentados no segundo capítulo indiquem que muitos dos espaços topológicos comumente encontrados na literatura sejam resolúveis ou possuam pontos isolados, asseguraremos, no terceiro capítulo desta dissertação, a existência de espaços topológicos irresolúveis e sem pontos isolados. A primeira seção tratará de diversos graus de irresolubilidade pertinentes a um espaço topológico, tais como maximalidade, submaximalidade, irresolubilidade hereditária e irresolubilidade hereditária por abertos. Um espaço topológico $(X, \tau)$ é dito maximal se é denso em si mesmo e toda topologia $\tau^{\prime}$ sobre o conjunto $X$ estritamente mais fina do que $\tau$ faz com que o espaço topológico $\left(X, \tau^{\prime}\right)$ possua pontos isolados. Se todo subconjunto denso de $X$ for aberto em $X$, diremos que o espaço topológico $(X, \tau)$ é submaximal. Todo espaço topológico maximal e $T_{1}$ é, em particular, submaximal. Evidentemente, todo espaço topológico submaximal é irresolúvel. A seção seguinte, desenvolverá a técnica de expansão de espaços topológicos, a qual nos permitirá construir um exemplo de espaço topológico completamente regular, hereditariamente irresolúvel e denso em si mesmo.

No capítulo 4, mostraremos que dado $n>1$ um número natural, é possível exibir um exemplo de espaço topológico regular que é $n$-resolúvel, mas que não é $(n+1)$-resolúvel. Provaremos, contudo, que se um espaço topológico for $n$-resolúvel, para todo número natural $n>1$, o mesmo também será $\omega$-resolúvel.

$\mathrm{Na}$ primeira seção do capítulo 5, apresentaremos a construção de um subespaço enumerável, denso e submaximal de $2^{\mathfrak{c}}$. Na segunda seção, mostraremos que o cubo de Cantor em questão não possui um subespaço denso maximal. 
No sexto e último capítulo desta dissertação, apresentaremos o conceito de espaço topológico $\mathcal{D}$-forçado. Demonstraremos que, para todo cardinal infinito $\kappa$, o cubo de Cantor $2^{2^{\kappa}}$ possui um subespaço denso e submaximal de cardinalidade $\kappa$. Provaremos, ainda, que dado um cardinal infinito $\mu$, existe um espaço topológico completamente regular que é $\mu$ resolúvel, mas que não é $\mu^{+}$-resolúvel. Mostraremos, por fim, que se $\lambda$ é um cardinal regular não enumerável, é possível exibir um espaço topológico que é $\mu$-resolúvel, para todo $\mu<\lambda$, mas que não é $\lambda$-resolúvel.

Demonstrou-se, em [3], que se $\lambda$ é um cardinal singular $\operatorname{com} \operatorname{cf}(\lambda)=\omega$ e se $X$ é um espaço topológico $\mu$-resolúvel, para todo $\mu<\lambda$, então $X$ é $\lambda$-resolúvel. Contudo, a seguinte questão permanece sem solução: se $\lambda$ é um cardinal singular com $\operatorname{cf}(\lambda)>\omega$ e se $X$ é um espaço topológico $\mu$-resolúvel, para todo $\mu<\lambda$, é verdade que $X$ é $\lambda$-resolúvel? 


\section{Capítulo 1}

\section{Definições e resultados preliminares}

Este capítulo tem por objetivo estabelecer a notação que será utilizada ao longo desta dissertação, além de destacar resultados preliminares e definições essenciais à compreensão do texto. A primeira seção será dedicada à Teoria dos Conjuntos e a segunda, à Topologia Geral. Nossas principais referências em Teoria dos Conjuntos são [10] e [13]; em Topologia Geral, citamos [1], [7], [11] e [15]. 


\subsection{Teoria dos Conjuntos}

Indicaremos por $\mathcal{P}(A)$ a coleção de todos os subconjuntos de um conjunto $A$. A união, a intersecção e a diferença de dois conjuntos $A$ e $B$ serão denotadas, respectivamente, por $A \cup B, A \cap B$ e $A \backslash B$.

As definições de ordinal (sucessor e limite) e cardinal (sucessor e limite), cofinalidade, ordinal (regular e singular) e cardinal (regular e singular) serão supostas conhecidas.

Se $X$ é um conjunto e $\lambda$ é um cardinal, definimos

$$
[X]^{\lambda}=\{A \in \mathcal{P}(X):|A|=\lambda\}
$$

e

$$
[X]^{<\lambda}=\{A \in \mathcal{P}(X):|A|<\lambda\}
$$

Dados $\mu$ e $\lambda$ cardinais, denotaremos a coleção das funções parciais finitas de $\mu$ em $\lambda$ por $\operatorname{Fn}(\mu, \lambda)=\left\{f: f\right.$ é uma função tal que $\operatorname{dom} f \in[\mu]^{<\omega}$ e $f(\zeta) \in \lambda$, para todo $\left.\zeta \in \operatorname{dom} f\right\}$.

Seja $X$ um conjunto não vazio. Dizemos que uma família $\mathcal{F}$ de subconjuntos de $X$ é um filtro sobre $X$ se as seguintes condições estão verificadas:

(1) $X \in \mathcal{F}$ e $\emptyset \notin \mathcal{F}$;

(2) Se $A \in \mathcal{F}$ e $B \in \mathcal{F}$, então $A \cap B \in \mathcal{F}$;

(3) Se $A, B \subset X, A \in \mathcal{F}$ e $A \subset B$, então $B \in \mathcal{F}$.

Proposição 1.1.1. Seja $X$ um conjunto infinito. O conjunto

$$
\mathcal{F}:=\{A \subset X: X \backslash A \text { é finito }\}
$$

é um filtro sobre $X$, chamado de filtro cofinito ou filtro de Fréchet. 
Demonstração. Evidentemente, $X \in \mathcal{F}$. Como $X$ é um conjunto infinito, concluímos que $\emptyset \notin \mathcal{F}$. Sejam $A, B \in \mathcal{F}$. Sabemos que $X \backslash(A \cap B)=(X \backslash A) \cup(X \backslash B)$. Por hipótese, $X \backslash A$ e $X \backslash B$ são conjuntos finitos, e, portanto, $X \backslash(A \cap B)$ também é um conjunto finito. Logo, $A \cap B \in \mathcal{F}$. Por fim, sejam $A, B \subset X$ tais que $A \in \mathcal{F}$ e $A \subset B$. Temos que $X \backslash B \subset X \backslash A$ e, como $X \backslash A$ é finito, segue que $X \backslash B$ também o é. Portanto, $B \in \mathcal{F}$.

Seja $\mathcal{A}$ uma família de conjuntos. Dizemos que $\mathcal{A}$ tem a propriedadade da intersecção finita se, para todo subconjunto finito e não vazio $\left\{A_{1}, \ldots, A_{n}\right\}$ de $\mathcal{A}$ tivermos que $A_{1} \cap \ldots \cap A_{n} \neq \emptyset$.

Afirmação 1.1.2. Todo filtro tem a propriedade da intersecção finita.

Proposição 1.1.3. Sejam $X$ um conjunto não vazio e $G \subset \mathcal{P}(X)$. Se $G$ tem a propriedade da intersecção finita, então existe um filtro $\mathcal{F}$ sobre $X$ tal que $G \subset \mathcal{F}$.

Demonstração. Seja $\mathcal{F}$ a coleção de todos os subconjuntos $Y$ de $X$ tais que existe uma coleção finita $H=\left\{Y_{1}, \ldots, Y_{n}\right\}$ de elementos de $G$ verificando $Y_{1} \cap \ldots \cap Y_{n} \subset Y$. Temos que $\mathcal{F}$ é um filtro sobre $X$ e que $G \subset \mathcal{F}$.

Dizemos que um filtro $\mathcal{F}$ sobre um conjunto não vazio $X$ é um ultrafiltro se não existe um filtro sobre $X$ que contenha $\mathcal{F}$ propriamente.

Proposição 1.1.4. Um filtro $\mathcal{F}$ sobre um conjunto não vazio $X$ é um ultrafiltro sobre $X$ se, e somente se, para todo subconjunto $A$ de $X$, vale que $A \in \mathcal{F}$ ou $X \backslash A \in \mathcal{F}$.

Demonstração. Consideremos $X$ um conjunto não vazio. Suponhamos, primeiramente, que $\mathcal{F}$ é um ultrafiltro sobre $X$. Seja $A$ um subconjunto de $X$. Se $A$ é tal que $A \cap F \neq \emptyset$, 
para todo $F \in \mathcal{F}$, então a família $\mathcal{F} \cup\{A\}$ goza da propriedade da intersecção finita e, portanto, existe um filtro $\mathcal{G}$ sobre $X$ tal que $\mathcal{F} \cup\{A\} \subset \mathcal{G}$. Como $\mathcal{F}$ é maximal, segue que $\mathcal{F}=\mathcal{G}$ e, portanto, $A \in \mathcal{F}$. Por outro lado, se existe $F \in \mathcal{F}$ tal que $A \cap F=\emptyset$, então $F \subset X \backslash A$ e, como $F \in \mathcal{F}$, decorre que $X \backslash A \in \mathcal{F}$.

Reciprocamente, suponhamos que $\mathcal{F}$ seja um filtro sobre $X$ tal que, para todo subconjunto $A$ de $X$, tenhamos que $A \in \mathcal{F}$ ou $X \backslash A \in \mathcal{F}$. Se $\mathcal{F}$ não fosse um ultrafiltro, existiria um filtro $\mathcal{G}$ sobre $X$ que conteria $\mathcal{F}$ propriamente. Portanto, existiria $A$ um subconjunto de $X$ tal que $A \in \mathcal{G}$ e $A \notin \mathcal{F}$. Por hipótese, $X \backslash A \in \mathcal{F}$. Como $\mathcal{F} \subset \mathcal{G}$, teríamos que $X \backslash A \in \mathcal{G}$, uma contradição.

Proposição 1.1.5. Seja $X$ um conjunto infinito. Se $\mathcal{F}$ é um ultrafiltro sobre $X$ e $B$ é um subconjunto de $X$ tal que $B \cap A \neq \emptyset$ para todo $A \in \mathcal{F}$, então $B \in \mathcal{F}$.

Demonstração. Suponhamos que $B \notin \mathcal{F}$. Como $\mathcal{F}$ é um ultrafiltro sobre $X$, concluímos que $X \backslash B \in \mathcal{F}$ e, portanto, $B \cap(X \backslash B) \neq \emptyset$, o que é absurdo. Logo, $B \in \mathcal{F}$.

Um ultrafiltro $\mathcal{F}$ sobre um conjunto infinito $X$ é dito livre se $\mathcal{F}$ contém o filtro cofinito.

Proposição 1.1.6. Sejam $X$ um conjunto infinito e $\mathcal{F}$ um ultrafiltro livre sobre $X$. Não existe $x \in X$ tal que $x \in A$, para todo $A \in \mathcal{F}$.

Demonstração. Seja $x$ um elemento qualquer de $X$. O conjunto $X \backslash\{x\}$ pertence ao filtro cofinito sobre $X$. Como $\mathcal{F}$ é um ultrafiltro livre sobre $X$ segue que $X \backslash\{x\} \in \mathcal{F}$ e, portanto, $x$ não pertence a todo elemento de $\mathcal{F}$. 
Teorema 1.1.7. Todo filtro sobre um conjunto não vazio $X$ se estende a um ultrafiltro.

Demonstração. Seja $\mathcal{F}_{0}$ um filtro sobre $X$. Consideremos $\mathcal{P}$ a coleção de todos os filtros $\mathcal{F}$ sobre $X$ tais que $\mathcal{F}_{0} \subset \mathcal{F}$, ordenada pela inclusão. Seja $\mathcal{C}$ uma cadeia em $\mathcal{P}$. Temos que $\bigcup \mathcal{C}$ é um filtro sobre $X$. Logo, $\bigcup \mathcal{C}$ é um majorante da cadeia $\mathcal{C}$. Do lema de Kuratowski-Zorn decorre que $\mathcal{P}$ possui um elemento maximal $\mathcal{U}$. Portanto, $\mathcal{U}$ é um ultrafiltro sobre $X$ que contém $\mathcal{F}_{0}$.

Corolário 1.1.8. Todo conjunto infinito admite um ultrafiltro livre.

Demonstração. Seja $X$ um conjunto infinito. Do teorema 1.1.7 segue que é possível estender o filtro cofinito a um ultrafiltro (livre) sobre $X$.

Uma família de conjuntos $\mathcal{A}$ é dita um $\Delta$-sistema se existe um conjunto $R$ tal que $A \cap B=R$, quaisquer que sejam $A$ e $B$ elementos distintos de $\mathcal{A}$. Neste caso, dizemos que $R$ é a raiz do $\Delta$-sistema $\mathcal{A}$.

Teorema 1.1.9. Sejam $\kappa$ um cardinal infinito e $\mathcal{A}$ uma família de conjuntos. Consideremos $\theta>\kappa$ um cardinal regular tal que, para todo $\alpha<\theta$, tenhamos $\left|\alpha^{<\kappa}\right|<\theta$. Suponhamos que $|\mathcal{A}| \geq \theta$ e que $|x|<\kappa$, para todo $x \in \mathcal{A}$. Então, existe $\mathcal{B} \subset \mathcal{A}$, com $|\mathcal{B}|=\theta$, tal que $\mathcal{B}$ forma um $\Delta$-sistema.

Demonstração. [13]. 
Lema (do $\Delta$-sistema) 1.1.10. Se $\mathcal{A}$ é uma família não enumerável de conjuntos finitos, existe uma subfamília não enumerável $\mathcal{B} \subset \mathcal{A}$ que forma um $\Delta$-sistema.

Se $\mathcal{A} \subset \mathcal{P}(\omega)$, dizemos que $\mathcal{A}$ é uma família independente se, para quaisquer $m, n \in$ $\omega \backslash\{0\}$ e quaisquer $A_{1}, \ldots, A_{m}, B_{1}, \ldots, B_{n}$ elementos distintos de $\mathcal{A}$, vale que

$$
\left|A_{1} \cap \ldots \cap A_{m} \cap\left(\omega \backslash B_{1}\right) \cap \ldots \cap\left(\omega \backslash B_{n}\right)\right|=\omega
$$

$\mathcal{A}$ é dita uma família independente maximal se $\mathcal{A}$ é uma família independente e é maximal com respeito a esta propriedade.

\subsection{Topologia Geral}

Um espaço topológico é um par $(X, \tau)$, onde $X$ é um conjunto e $\tau$ é uma coleção de subconjuntos de $X$ satisfazendo as seguintes propriedades:

(1) $\emptyset \in \tau$ e $X \in \tau$;

(2) Se $U, V \in \tau$, então $U \cap V \in \tau$;

(3) Se $\mathcal{A} \subset \tau$, então $\cup \mathcal{A} \in \tau$.

Neste caso, dizemos que $\tau$ é uma topologia sobre $X$ e que $X$ é o suporte do espaço topológico $(X, \tau)$. Os elementos do conjunto $X$ são denominados pontos de $(X, \tau)$. Quando não houver dúvidas a respeito da topologia $\tau$ dada a um conjunto $X$, denotaremos o espaço topológico $(X, \tau)$ por $X$, simplesmente.

Assumiremos conhecidos alguns conceitos básicos de Topologia Geral (abertos e fechados, interior e fecho, subconjuntos densos, pontos isolados e de acumulação, sistemas 
fundamentais de vizinhanças, base e subbase de um espaço topológico, funções contínuas e homeomorfismos, compactos, conexos, espaços métricos e metrizáveis).

Se $X$ é um espaço topológico e $A$ é um subconjunto de $X$, denotaremos por $\operatorname{int}_{X}(A)$ o interior de $A$ em $X$ e por $\operatorname{cl}_{X}(A)$ o fecho (ou aderência) de $A$ em $X$. Quando não houver dúvidas a respeito do espaço topológico considerado, escreveremos int $(A)$, em vez de $\operatorname{int}_{X}(A)$ e $\bar{A}$, em vez $\operatorname{de~} \mathrm{cl}_{X}(A)$.

Dizemos que um espaço topológico $X$ é discreto, se todo subconjunto de $X$ for aberto em $X$. Dado um conjunto infinito $X$, a topologia cofinita sobre $X$ é definida por

$$
\{\emptyset\} \cup\{A \subset X:|X \backslash A|<\omega\} .
$$

Um espaço topológico $X$ é dito denso em si mesmo se $X$ não possui pontos isolados.

Dados um cardinal $\kappa$ e um espaço topológico $X$, um subconjunto $D$ de $X$ é dito $\kappa$-denso em $X$ se $|D \cap U| \geq \kappa$, para todo aberto não vazio $U$ de $X$. Portanto, um subconjunto $D$ de $X$ é denso em $X$ se, e somente se, $D$ é 1-denso em $X$.

Seja $X$ um espaço topológico. Dizemos que $X$ satisfaz o primeiro axioma de enumerabilidade (respectivamente, o segundo axioma de enumerabilidade) se todo ponto de $X$ admite um sistema fundamental de vizinhanças enumerável (respectivamente, se $X$ possui uma base de abertos enumerável).

As funções cardinais mencionadas ao longo do texto encontram-se listadas abaixo.

O peso de um espaço topológico $X$ é denotado por $w(X)$ e é definido por

$$
w(X)=\min \{|\mathcal{B}|: \mathcal{B} \text { é uma base de abertos de } X\} .
$$

A densidade de um espaço topológico $X$ é denotada por $d(X)$ e é definida por

$$
d(X)=\min \{|D|: D \text { é um subconjunto denso de } X\} .
$$

O caráter de um espaço topológico $X$ é denotado por $\chi(X)$ e é definido por

$$
\chi(X)=\sup \{\chi(p, X): p \in X\}
$$


onde $\chi(p, X)$ é o caráter de $p$ em $X$, dado por

$$
\chi(p, X)=\min \{|\mathcal{V}|: \mathcal{V} \text { é um sistema fundamental de vizinhanças de } x \text { em } X\} \text {. }
$$

Seja $X$ um espaço topológico. Uma coleção de subconjuntos abertos dois a dois disjuntos de $X$ é denominada família celular de $X$. A celularidade do espaço topológico $X$ é denotada por $c(X)$ e é definida por

$$
c(X)=\sup \{|\mathcal{C}|: \mathcal{C} \text { é uma família celular de } X\} .
$$

Definimos, ainda,

$$
\hat{c}(X)=\min \{\lambda: \lambda>|\mathcal{C}|, \text { se } \mathcal{C} \text { é uma família celular de } X\} .
$$

Embora diversos textos de Topologia Geral admitam, por hipótese, que as funções cardinais apresentadas acima somente tomem valores infinitos, não faremos tal suposição nesta dissertação.

Consideremos $X$ um espaço topológico.

Dizemos que $X$ é um espaço topológico $T_{0}$ (ou que $X$ satisfaz o axioma de separação de Kolmogoroff ) se dados dois pontos distintos quaisquer de $X$, existe um subconjunto aberto de $X$ que contém apenas um destes pontos.

Dizemos que $X$ é um espaço topológico $T_{1}$ (ou que $X$ satisfaz o axioma de separação de Riesz) se dados $x$ e $y$ pontos distintos quaisquer de $X$, existem subconjuntos abertos $U$ e $V$ de $X$ tais que $x \in U$ e $y \notin U$ e tais que $x \notin V$ e $y \in V$.

Observamos que $X$ é um espaço topológico $T_{1}$ se, e somente se, $\{x\}$ é um subconjunto fechado de $X$, para todo $x \in X$.

Dizemos que $X$ é um espaço topológico $T_{2}$ (ou que $X$ é um espaço de Hausdorff) se dados $x$ e $y$ pontos distintos quaisquer de $X$, existem subconjuntos abertos $U$ e $V$ de $X$ tais que $x \in U, y \in V$ e $U \cap V=\emptyset$. 
Dizemos que $X$ é um espaço topológico $T_{2 \frac{1}{2}}$ (ou que $X$ é um espaço de Urysohn) se dados $x$ e $y$ pontos distintos quaisquer de $X$, existem subconjuntos abertos $U$ e $V$ de $X$ tais que $x \in U, y \in V$ e $\bar{U} \cap \bar{V}=\emptyset$.

Dizemos que $X$ é um espaço topológico $T_{3}$ se dados $x \in X$ e $F$ um subconjunto fechado de $X$ tal que $x \notin F$, existem abertos $U$ e $V$ de $X$ tais que $x \in U, F \subset V$ e $U \cap V=\emptyset$. Um espaço topológico que é $T_{1}$ e $T_{3}$ é dito regular.

Observamos que um espaço topológico $X$ é $T_{3}$ se, e só se, para cada ponto $x$ de $X$ e cada vizinhança aberta $V$ de $x$ em $X$, existe um subconjunto aberto $U$ de $X$ tal que $x \in U \subset \bar{U} \subset V$.

Dizemos que $X$ é um espaço topológico $T_{3 \frac{1}{2}}$ se, para cada ponto $x$ de $X$ e cada fechado $F$ de $X$ ao qual $x$ não pertence, existe uma função contínua $f: X \rightarrow[0,1]$ tal que $f(x)=0$ e $f(F) \subset\{1\}$. Um espaço topológico que é $T_{1}$ e $T_{3 \frac{1}{2}}$ é dito completamente regular ou um espaço de Tychonoff.

Dizemos que $X$ é um espaço topológico $T_{4}$ se, para cada par de fechados disjuntos $F$ e $G$ de $X$, existem subconjuntos abertos $U$ e $V$ de $X$ tais que $F \subset U, G \subset V$ e $U \cap V=\emptyset$. Um espaço topológico $T_{1}$ e $T_{4}$ é dito normal.

Se um subconjunto $A$ de um espaço topológico $X$ for tal que $A=\operatorname{int}(\bar{A})$, diremos que $A$ é um aberto regular de $X$. Um espaço topológico $X$ é dito semi-regular se os abertos regulares de $X$ constituem uma base para $X$.

Proposição 1.2.1. Todo espaço topológico regular é semi-regular.

Demonstração. Consideremos $X$ um espaço topológico regular. Sejam $V$ um subconjunto aberto não vazio de $X$ e $x$ um ponto de $V$. Como $X$ é regular, existe um aberto $U$ de $X$ tal que $x \in U \subset \bar{U} \subset V$. Claramente, $U \subset \operatorname{int}(\bar{U}) \subset \bar{U}$ e, portanto, $x \in \operatorname{int}(\bar{U}) \subset V$. Como $\operatorname{int}(\bar{U})$ é um aberto regular de $X$, segue que $X$ é semi-regular. 
Se $(X, \tau)$ é um espaço topológico e $Y$ é um subconjunto de $X$, a topologia induzida por $X$ sobre $Y$ é dada por $\tau^{\prime}=\{Y \cap U: U \in \tau\}$. Em algumas ocasiões, denotaremos $\tau^{\prime}$ por $\tau \uparrow_{Y}$.

Se $\left\{X_{i}: i \in I\right\}$ é uma família de espaços topológicos, consideraremos o produto cartesiano $\prod_{i \in I} X_{i}$ munido da topologia gerada pela base de abertos $\left\{\prod_{i \in I} U_{i}: U_{i}\right.$ é aberto em $X_{i}$, para todo $i \in I$ e $\left.\left|\left\{i \in I: U_{i} \neq X_{i}\right\}\right|<\omega\right\}$.

Teorema (de Hewitt-Marczewski-Pondiczery) 1.2.2. Seja $\left\{X_{i}: i \in I\right\}$ uma família de espaços topológicos. Se $d\left(X_{i}\right) \leq \kappa \geq \omega$, para todo $i \in I$ e $|I| \leq 2^{\kappa}$, então $d\left(\prod_{i \in I} X_{i}\right) \leq \kappa$.

Demonstração. [7].

Se um subconjunto $A$ de um espaço topológico $X$ for aberto e fechado em $X$, diremos que $A$ é um aberto-fechado de $X$. Um espaço topológico $X$ é dito zero-dimensional se possui uma base de abertos-fechados de $X$.

Proposição 1.2.3. Se $X$ é um espaço topológico $T_{1}$ e zero-dimensional, então $X$ é completamente regular.

Demonstração. Sejam $x$ um ponto de $X$ e $F$ um subconjunto fechado de $X$ ao qual $x$ não pertence. Desta forma, $X \backslash F$ é uma vizinhança aberta de $x$ em $X$. Como $X$ é zero dimensional, existe um subconjunto aberto-fechado $U$ de $X$ tal que $x \in U \subset X \backslash F$. Seja $f: X \rightarrow[0,1]$ tal que $f(U) \subset\{0\}$ e $f(X \backslash U) \subset\{1\}$. Como $f$ é contínua, segue que $X$ é $T_{3 \frac{1}{2}}$. Como $X$ é $T_{1}$, por hipótese, concluímos que $X$ é completamente regular. 
Um espaço topológico $X$ é dito localmente compacto se todo ponto de $X$ possui um sistema fundamental de vizinhanças compactas.

Sabemos que se $X$ é um espaço topológico $T_{2}$, então $X$ é localmente compacto se, e somente se, dados $x \in X$ e $U$ uma vizinhança de $x$ em $X$, existe $V$ uma vizinhança de $x$ em $X$ tal que $\bar{V}$ é um subconjunto compacto de $U$. Portanto, todo espaço topológico compacto e $T_{2}$ é localmente compacto, bem como todo subconjunto aberto de um espaço topológico localmente compacto e $T_{2}$.

Teorema 1.2.4. Se $X$ é um espaço topológico localmente compacto e $T_{2}$, então $\omega(X) \leq|X|$.

Demonstração. [7]. 


\section{Capítulo 2}

\section{Espaços topológicos resolúveis}

$\mathrm{Na}$ primeira seção deste capítulo, introduziremos a definição de espaço topológico resolúvel e mostraremos, a princípio, que todo espaço métrico sem pontos isolados goza desta propriedade. Provaremos que se o peso de um espaço topológico $T_{0}$ e sem pontos isolados for menor ou igual ao seu caráter de dispersão, o mesmo será resolúvel. Encerraremos esta seção demonstrando que todo espaço topológico localmente compacto, $T_{2}$ e denso em si mesmo é resolúvel, bem como todo espaço topológico $T_{0}$, sem pontos isolados e que satisfaz o segundo axioma de enumerabilidade. Todos os resultados mencionados acima encontramse em [8]. Na seção seguinte, apresentaremos a noção de $\kappa$-resolubilidade de um espaço topológico, onde $\kappa>1$ é um cardinal. Destacaremos, em particular, o conceito de espaço topológico maximalmente resolúvel e generalizaremos, seguindo [4], os principais resultados apresentados na seção anterior. 


\subsection{A noção de resolubilidade}

Definição 2.1.1. Um espaço topológico $(X, \tau)$ é dito resolúvel se o mesmo possui dois subconjuntos densos complementares. Caso contrário, $X$ é dito irresolúvel.

Observamos que se um espaço topológico possui um ponto isolado, o mesmo é irresolúvel. Com efeito, se $p$ é um ponto isolado de um espaço topológico $X$, então $\{p\}$ é um subconjunto aberto de $X$, o que faz com que o ponto $p$ pertença a todo subconjunto denso de $X$.

A reta real, munida de sua topologia usual, é um exemplo de espaço topológico resolúvel. De fato, o conjunto dos números racionais e o seu complementar - o conjunto dos números irracionais - são ambos densos no espaço topológico em questão. De maneira mais geral, dado $n$ um número inteiro positivo, o espaço euclidiano $n$-dimensional $\mathbb{R}^{n}$, munido de sua topologia usual, possui dois subconjuntos densos complementares (a saber, o conjunto das $n$-uplas de números reais onde cada coordenada é um número racional e o conjunto das $n$-uplas de números reais onde pelo menos uma coordenada é um número irracional).

Teorema 2.1.2. Todo espaço métrico sem pontos isolados é resolúvel.

Demonstração. Sejam $(M, d)$ um espaço métrico sem pontos isolados e $\left\{p_{\alpha}\right\}_{\alpha<|M|}$ uma indexação do conjunto $M$. Definiremos, por indução transfinita, dois subconjuntos complementares $A$ e $B$ de $M$, ambos densos no espaço métrico em questão. Daí resultará que $M$, munido da topologia associada à métrica $d$, é um espaço topológico resolúvel.

$\mathrm{O}$ ponto $p_{0}$ será um elemento do conjunto $A$ e o ponto $p_{1}$, do conjunto $B$. Seja $1<\alpha<|M|$ um ordinal e suponhamos que, para todo ordinal $\beta<\alpha$, o ponto $p_{\beta}$ já tenha sido designado a algum dos conjuntos $A$ ou $B$. Denotemos por $A_{\alpha}$ o conjunto dos pontos $p_{\beta}$, com $\beta<\alpha$, que sejam elementos do conjunto $A$ e, analogamente, denotemos por $B_{\alpha}$ o conjunto dos pontos $p_{\beta}$, com $\beta<\alpha$, que sejam elementos do conjunto $B$. 
Se $d\left(p_{\alpha}, A_{\alpha}\right)<d\left(p_{\alpha}, B_{\alpha}\right)$, o ponto $p_{\alpha}$ pertencerá ao conjunto $B$.

Se $d\left(p_{\alpha}, A_{\alpha}\right) \geq d\left(p_{\alpha}, B_{\alpha}\right)$, o ponto $p_{\alpha}$ pertencerá ao conjunto $A$.

Temos, portanto, que $M=A \cup B$ e que $A \cap B=\emptyset$.

Afirmamos ainda que $A$ e $B$ são subconjuntos densos de $M$. De fato, suponhamos por absurdo que $A$ não seja denso em $M$. Existe, portanto, um aberto não vazio $U$ de $M$ tal que $U \cap A=\emptyset$, ou seja, tal que $U \subset M \backslash A=B$. Seja $p_{\alpha} \in U$ tal que se $\beta<\alpha$, então $p_{\beta} \notin U$. Como $U$ é aberto em $M$, existe $\epsilon>0$ tal que $B_{\epsilon}\left(p_{\alpha}\right) \subset U$. Como $M$ não tem pontos isolados, existe $\beta>\alpha$ tal que $p_{\beta} \in B_{\frac{\epsilon}{2}}\left(p_{\alpha}\right)$. Uma vez que $p_{\alpha} \in B_{\beta}$, segue que $d\left(p_{\beta}, B_{\beta}\right)<\frac{\epsilon}{2}$. Como $p_{\beta} \in B$, temos que $d\left(p_{\beta}, A_{\beta}\right)<d\left(p_{\beta}, B_{\beta}\right)<\frac{\epsilon}{2}$. Assim, existe $p_{\gamma} \in A_{\beta} \subset A$ tal que $d\left(p_{\beta}, p_{\gamma}\right)<\frac{\epsilon}{2}$. Portanto, $d\left(p_{\alpha}, p_{\gamma}\right) \leq d\left(p_{\alpha}, p_{\beta}\right)+d\left(p_{\beta}, p_{\gamma}\right)<\frac{\epsilon}{2}+\frac{\epsilon}{2}=\epsilon$. Logo, $p_{\gamma} \in B_{\epsilon}\left(p_{\alpha}\right) \subset U \subset B$, o que é absurdo, pois $p_{\gamma} \in A$ e $A \cap B=\emptyset$. Logo, $A$ é um subconjunto denso de $M$.

Mostramos, de maneira similar, que $B$ é, também, um subconjunto denso de $M$ e, portanto, $M$ é resolúvel.

Um outro exemplo de espaço topológico resolúvel é dado por um conjunto infinito $X$, munido da topologia cofinita. Com efeito, $|X| \geq \omega$ e, portanto, $X$ contém um subconjunto $A$ tal que $|A|=|X \backslash A|=|X|$. Como todo aberto não vazio de $X$ possui complemento finito no espaço em questão, segue que $A$ e $X \backslash A$ são, ambos, densos em $X$. Notemos, finalmente, que um conjunto infinito munido da topologia cofinita é um espaço topológico que não é $T_{2}$ e, portanto, é um espaço topológico resolúvel que não é metrizável.

A próxima proposição apresenta uma caracterização bastante simples dos espaços topológicos resolúveis.

Proposição 2.1.3. Seja X um espaço topológico. As seguintes afirmações são equivalentes: 
(1) X é resolúvel;

(2) X contém dois subconjuntos densos disjuntos;

(3) X contém um subconjunto denso com interior vazio;

(4) X contém dois subconjuntos complementares, ambos com interior vazio.

Demonstração. A implicação (1) $\Rightarrow(2)$ é imediata. A fim de mostrar que (2) $\Rightarrow(3)$, suponhamos que existam $D_{1}, D_{2}$ subconjuntos densos e disjuntos de $X$. Afirmamos que $D_{2}$ tem interior vazio em $X$. Com efeito, se existisse $U$ um aberto não vazio de $X$ contido em $D_{2}$, teríamos que $D_{1} \cap U \subset D_{1} \cap D_{2}=\emptyset$, o que contradiz o fato de $D_{1}$ ser denso em $X$. Portanto, $D_{2}$ é um subconjunto denso de $X$ com interior vazio em $X$.

Suponhamos, agora, que exista $D$ um subconjunto denso de $X$, com interior vazio. Mostremos que $X \backslash D$ também tem interior vazio em $X$. Com efeito, se existisse um aberto não vazio $U$ de $X$ tal que $U \subset X \backslash D$, teríamos que $U \cap D=\emptyset$, o que é absurdo, posto que $D$ é denso em $X$. Logo, (3) $\Rightarrow(4)$.

Mostremos, finalmente, que (4) $\Rightarrow(1)$. Por hipótese, existe $D \subset X$ tal que $\operatorname{int}(D)=$ $\operatorname{int}(X \backslash D)=\emptyset$. Afirmamos que $D$ e $X \backslash D$ são densos em $X$. Com efeito, seja $U$ um subconjunto aberto não vazio de $X$, qualquer. Como $\operatorname{int}(D)=\operatorname{int}(X \backslash D)=\emptyset$, segue que $U \not \subset D$ e $U \not \subset X \backslash D$. Portanto, $U \cap(X \backslash D) \neq \emptyset$ e $U \cap D \neq \emptyset$, o que mostra que $D$ e $X \backslash D$ são densos em $X$ e, desta forma, $X$ é resolúvel.

Sabemos que se um espaço topológico é resolúvel, então o mesmo possui dois subconjuntos densos disjuntos. Em particular, tais subconjuntos densos têm interiores disjuntos. O objetivo do nosso próximo resultado é mostrar que esta condição é, também, suficiente para assegurar a resolubilidade de um espaço topológico. 
Proposição 2.1.4. Se $X$ é um espaço topológico que contém dois subconjuntos densos $A$ e $B$, tais que

$$
\operatorname{int}(A) \cap \operatorname{int}(B)=\emptyset
$$

então $X$ é resolúvel.

Demonstração. Seja $X$ um espaço topológico nas condições do enunciado. Mostraremos que $X$ contém um subconjunto denso com interior vazio. Para tanto, consideremos

$$
C=[\overline{(X \backslash A)} \cap A] \cup[\operatorname{int}(A) \cap B] .
$$

Provemos, primeiramente, que $C$ é um subconjunto denso de $X$. Para tanto, seja $U$ um aberto não vazio de $X$.

Se $U \cap \operatorname{int}(A) \neq \emptyset$, então $U \cap[\operatorname{int}(A) \cap B] \neq \emptyset$, pois $U \cap \operatorname{int}(A)$ é um subconjunto aberto não vazio de $X$ e $B$ é um subconjunto denso de $X$. Logo, $C \cap U \neq \emptyset$.

Se $U \cap \operatorname{int}(A)=\emptyset$, então $U \subset X \backslash \operatorname{int}(A)=X \backslash[X \backslash \overline{(X \backslash A)}]=\overline{(X \backslash A)}$. Como $A$ é um subconjunto denso de $X$, segue que $U \cap A \neq \emptyset$. Portanto, $U \cap[\overline{(X \backslash A)} \cap A]=U \cap A \neq \emptyset$. Logo, $C \cap U \neq \emptyset$.

Portanto, $C$ é um subconjunto denso em $X$.

Afirmamos, ainda, que $C$ tem interior vazio em $X$. De fato, se existisse $U$ um subconjunto aberto não vazio de $X$ tal que $U \subset C$, teríamos que $U \subset A$, uma vez que $\overline{(X \backslash A)} \cap A \subset A, \operatorname{int}(A) \cap B \subset A$ e, portanto, $C \subset A . \quad$ Logo, $U \subset \operatorname{int}(A)$. Como $\operatorname{int}(A) \cap[\overline{(X \backslash A)} \cap A]=\emptyset$, segue que $U \subset \operatorname{int}(A) \cap B$. Em particular, $U \subset B$ e, portanto, $U \subset \operatorname{int}(B) . \operatorname{Logo}, U \subset \operatorname{int}(A) \cap \operatorname{int}(B)=\emptyset$, um absurdo.

Portanto, $C$ tem interior vazio em $X$.

Da proposição 2.1.3, segue que $X$ é um espaço topológico resolúvel. 
Quando um subconjunto $Y$ de um espaço topológico $X$ for resolúvel na topologia induzida diremos, simplesmente, que $Y$ é um subespaço resolúvel de $X$.

Proposição 2.1.5. Sejam $X$ um espaço topológico e $Y$ um subespaço resolúvel de $X$. Então $\bar{Y}$ é, também, um subespaço resolúvel de $X$.

Demonstração. Sejam $X$ um espaço topológico e $Y$ um subespaço resolúvel de $X$, com subconjuntos densos e complementares $D_{1}$ e $D_{2}$. Afirmamos que $D_{1}$ e $D_{2}$ são, também, subconjuntos densos de $\bar{Y}$.

Com efeito, seja $V$ um aberto não vazio de $\bar{Y}$. Existe, portanto, um subconjunto aberto $U$ de $X$ tal que $V=\bar{Y} \cap U$. Seja $p \in V$. Como $p \in \bar{Y}$ e $U$ é um aberto de $X$ ao qual $p$ pertence, segue que $U \cap Y$ é um aberto não vazio de $Y$ e, portanto, $D_{1} \cap(U \cap Y) \neq \emptyset$ e $D_{2} \cap(U \cap Y) \neq \emptyset$. Em particular, temos que $D_{1} \cap(U \cap \bar{Y}) \neq \emptyset$ e $D_{2} \cap(U \cap \bar{Y}) \neq \emptyset$, pois $Y \subset \bar{Y}$. Logo, $D_{1} \cap V \neq \emptyset$ e $D_{2} \cap V \neq \emptyset$. Daí, segue que $D_{1}$ e $D_{2}$ são subconjuntos densos e disjuntos de $\bar{Y}$. Da proposição 2.1 .3 concluímos que $\bar{Y}$ é resolúvel.

Corolário 2.1.6. Se X é um espaço topológico que contém um subconjunto denso resolúvel na topologia induzida, então $X$ é resolúvel.

Demonstração. Decorre imediatamente da proposição 2.1.5.

Proposição 2.1.7. Todo subconjunto aberto de um espaço topológico resolúvel é resolúvel na topologia induzida.

Demonstração. Sejam $X$ um espaço topológico resolúvel e $U$ um subconjunto aberto não vazio de $X$. Mostremos que $U$, munido da topologia induzida, é um espaço topológico resolúvel. 
Como $X$ é resolúvel, existem $D_{1}$ e $D_{2}$ subconjuntos densos e complementares de $X$. Afirmamos que $D_{1} \cap U$ e $D_{2} \cap U$ são subconjuntos densos (e, obviamente, complementares) de $U$. Com efeito, seja $V$ um aberto não vazio qualquer de $U$. Sabemos que existe $W$ um subconjunto aberto de $X$ tal que $V=W \cap U$. Portanto, $V$ é, também, aberto em $X$. Como $D_{1}$ e $D_{2}$ são subconjuntos densos de $X$, segue que $D_{1} \cap V \neq \emptyset$ e $D_{2} \cap V \neq \emptyset$. Como $V \subset U$, temos que $\left(D_{1} \cap U\right) \cap V \neq \emptyset$ e $\left(D_{2} \cap U\right) \cap V \neq \emptyset$. Logo, $D_{1} \cap U$ e $D_{2} \cap U$ são subconjuntos densos e complementares de $U$, o que nos permite concluir que $U$ é um subespaço resolúvel de $X$.

Proposição 2.1.8. Seja X um espaço topológico. Suponhamos que

$$
X=\bigcup_{i \in I} X_{i}
$$

onde $\left\{X_{i}\right\}_{i \in I}$ é uma família de subespaços resolúveis de X. Então, X é um espaço topológico resolúvel.

Demonstração. Seja $\mathcal{M}$ uma família maximal de subespaços resolúveis de $X$, dois a dois disjuntos. Afirmamos que $\bigcup \mathcal{M}$ é um subconjunto denso de $X$. Com efeito, se isto não ocorresse,

$$
U: = X \backslash \longdiv { \bigcup \mathcal { M } }
$$

seria um subconjunto aberto não vazio de $X$. Como

$$
X=\bigcup_{i \in I} X_{i}
$$

existe $i \in I$ tal que $U \cap X_{i} \neq \emptyset$.

Da proposição 2.1.7 decorre que $U \cap X_{i}$ é um subespaço resolúvel de $X_{i}$ e, portanto, $U \cap X_{i}$ é um subespaço resolúvel de $X$. 
Logo, $\mathcal{M} \cup\left\{U \cap X_{i}\right\}$ é uma família de subespaços resolúveis de $X$, dois a dois disjuntos, que contém $\mathcal{M}$ propriamente, o que contradiz a maximalidade de $\mathcal{M}$. Portanto, $\bigcup \mathcal{M}$ é um subconjunto denso de $X$.

Para cada $Y \in \mathcal{M}$, tomemos $\left\{D_{1}^{Y}, D_{2}^{Y}\right\}$ uma família de subconjuntos densos disjuntos de $Y$. Consideremos

$$
D_{1}:=\bigcup_{Y \in \mathcal{M}} D_{1}^{Y} \text { e } D_{2}:=\bigcup_{Y \in \mathcal{M}} D_{2}^{Y} .
$$

Afirmamos que $D_{1}$ e $D_{2}$ são subconjuntos densos e disjuntos de $X$. De fato, se houvesse

$$
p \in D_{1} \cap D_{2}=\left(\bigcup_{Y \in \mathcal{M}} D_{1}^{Y}\right) \cap\left(\bigcup_{Y \in \mathcal{M}} D_{2}^{Y}\right)
$$

existiriam $Y_{1}, Y_{2} \in \mathcal{M}$ tais que

$$
p \in D_{1}^{Y_{1}} \cap D_{2}^{Y_{2}} \subset Y_{1} \cap Y_{2}
$$

o que é um absurdo, já que os elementos de $\mathcal{M}$ são dois a dois disjuntos. Portanto, $D_{1} \cap D_{2}=\emptyset$.

Resta mostrar que, para cada $i \in\{1,2\}, D_{i}$ é um subconjunto denso de $X$. Para tanto, consideremos $U$ um aberto não vazio de $X$. Como $\bigcup \mathcal{M}$ é densa em $X$, temos que $U \cap(\bigcup \mathcal{M}) \neq \emptyset$. Portanto, existe $Y \in \mathcal{M}$ tal que $U \cap Y \neq \emptyset$. Logo, $U \cap Y$ é um subconjunto aberto não vazio de $Y$ e, portanto, $D_{i}^{Y} \cap(U \cap Y) \neq \emptyset$, já que $D_{i}^{Y}$ é denso em $Y$. Em particular, segue que $D_{i}^{Y} \cap U \neq \emptyset$ e, portanto, $D_{i} \cap U \neq \emptyset$. Logo, $D_{i}$ é denso em $X$.

Da proposição 2.1.3 segue que $X$ é resolúvel.

Teorema 2.1.9. Um espaço topológico $(X, \tau)$ é resolúvel se, e somente se, todo subespaço aberto não vazio de $X$ contém um subconjunto não vazio, que é resolúvel na topologia induzida. 
Demonstração. Da proposição 2.1.7 segue que se $X$ é um espaço topológico resolúvel, então todo subconjunto aberto não vazio $U$ de $X$ contém um subconjunto não vazio, que é resolúvel na topologia induzida - a saber, o próprio $U$.

Reciprocamente, consideremos $(X, \tau)$ um espaço topológico tal que todo subespaço aberto de $X$ contém um subconjunto não vazio e resolúvel na topologia induzida. Mostremos que $X$ é resolúvel.

De fato, consideremos $U_{0}$ um aberto não vazio de $X$ e $A_{0}$ um subconjunto não vazio de $U_{0}$, resolúvel na topologia induzida, com subconjuntos densos e complementares $D_{0}$ e $E_{0}$. Se $U_{1}=X \backslash \bar{A}_{0}$ for um aberto não vazio de $X$, existirá $A_{1}$ um subconjunto não vazio de $U_{1}$, resolúvel na topologia induzida, com subconjuntos densos e complementares $D_{1}$ e $E_{1}$.

Dado um ordinal $\alpha<|\tau|$, suponhamos construídos, para todo ordinal $\beta<\alpha$, subconjuntos $A_{\beta}$ de $X$, resolúveis na topologia induzida, com subconjuntos densos e complementares $D_{\beta}$ e $E_{\beta}$.

Consideremos

$$
U_{\alpha}:=X \backslash \overline{\left(\bigcup_{\beta<\alpha} A_{\beta}\right)} .
$$

Se $U_{\alpha} \neq \emptyset$, existirá $A_{\alpha} \subset U_{\alpha}$, resolúvel na topologia induzida, com subconjuntos densos e complementares $D_{\alpha}$ e $E_{\alpha}$. Repetindo este processo indutivamente, encontraremos um ordinal $\alpha_{0}$ tal que

$$
\overline{\bigcup_{\alpha<\alpha_{0}} A_{\alpha}}=X
$$

Sejam

$$
D:=\bigcup_{\alpha<\alpha_{0}} D_{\alpha} \text { e } E:=\bigcup_{\alpha<\alpha_{0}} E_{\alpha} .
$$

É evidente que $D$ e $E$ são subconjuntos disjuntos de $X$, já que, para todo $\alpha<\alpha_{0}$, vale $D_{\alpha} \cap E_{\alpha}=\emptyset \mathrm{e}$

$$
A_{\alpha} \subset X \backslash \overline{\left(\bigcup_{\beta<\alpha} A_{\beta}\right)}
$$


Além disso, $D$ e $E$ são subconjuntos densos de $X$. Com efeito, dado $V$ um aberto não vazio qualquer de $X$, temos que

$$
\emptyset \neq V=V \cap X=V \cap \overline{\left(\bigcup_{\alpha<\alpha_{0}} A_{\alpha}\right)} .
$$

Como $V$ é aberto em $X$, segue que

$$
V \cap\left(\bigcup_{\alpha<\alpha_{0}} A_{\alpha}\right) \neq \emptyset
$$

Logo, existe $\alpha<\alpha_{0}$ tal que $V \cap A_{\alpha} \neq \emptyset$ e, portanto

$$
D_{\alpha} \cap\left(V \cap A_{\alpha}\right) \neq \emptyset \quad \text { e } \quad E_{\alpha} \cap\left(V \cap A_{\alpha}\right) \neq \emptyset .
$$

Assim, $D_{\alpha} \cap V \neq \emptyset$ e $E_{\alpha} \cap V \neq \emptyset$. Portanto, $D \cap V \neq \emptyset$ е $E \cap V \neq \emptyset$.

Logo, $D$ e $E$ são subconjuntos densos de $X$.

Da proposição 2.1.3 segue que $X$ é resolúvel.

Definição 2.1.10. O caráter de dispersão de um espaço topológico $X$ é denotado por $\Delta(X)$ e definido por

$$
\Delta(X)=\min \{|U|: U \text { é um aberto não vazio de } X\}
$$

Lema 2.1.11. Seja $X$ um espaço topológico $T_{0}$. Todo subconjunto aberto, finito e não vazio de $X$ possui um ponto isolado.

Demonstração. Consideremos $U=\left\{p_{1}, \ldots, p_{n}\right\}$ um subconjunto aberto, finito e não vazio de $X$. Como $X$ é um espaço topológico $T_{0}$, é possível encontrar um subconjunto aberto $V$ de $X$ tal que $p_{1} \in V$ e $p_{2} \notin V$, ou tal que $p_{1} \notin V$ e $p_{2} \in V$. Ajustando convenientemente 
a notação, se necessário, podemos supor que $p_{1} \notin V$ e $p_{2} \in V$. Portanto, $U \cap V$ é um subconjunto aberto não vazio de $X$ que contém, no máximo, $n-1$ pontos. Repetindo este processo, encontraremos um subconjunto aberto de $X$ contido em $U$ que consiste de um único ponto.

Em particular, observamos que todo espaço topológico $T_{0}$, finito e não vazio possui um ponto isolado.

Corolário 2.1.12. Todo espaço topológico não vazio e $T_{0}$ tem caráter de dispersão infinito ou igual a 1.

Demonstração. Seja $X$ um espaço topológico não vazio e $T_{0}$, com caráter de dispersão finito. Mostremos que $\Delta(X)=1$. Com efeito, seja $U$ um subconjunto aberto de $X$ tal que $|U|=\Delta(X)$. Do lema 2.1.11 segue que $U$ possui um ponto isolado $p$, ou seja, que $\{p\}=U \cap V$, onde $V$ é um subconjunto aberto de $X$. Logo, $\{p\}$ é, também, aberto em $X$ e, portanto, $\Delta(X)=1$.

Teorema 2.1.13. Se X é um espaço topológico $T_{0}$ e sem pontos isolados tal que

$$
w(X) \leq \Delta(X)
$$

então $X$ é resolúvel.

Demonstração. Consideremos $\mathcal{B}$ uma base de abertos de $X$, tal que $|\mathcal{B}|=w(X)$. A fim de mostrar que $X$ é um espaço topológico resolúvel, é suficiente exibir dois subconjuntos disjuntos de $X$ que intersectam todo elemento de $\mathcal{B}$. 
Seja $\left\{U_{i}\right\}_{i<|\mathcal{B}|}$ uma indexação de $\mathcal{B}$. Definiremos dois subconjuntos disjuntos $A$ e $B$ de $X$ da seguinte maneira: escolhemos um ponto $p_{1} \in U_{1}$ e o designamos a $A$. Escolhemos, em seguida, um ponto $q_{1} \in U_{1}, q_{1} \neq p_{1}$ e o designamos a $B$. Note que é possível efetuar tal escolha, uma vez que $\left|U_{1}\right| \geq \omega$ (pois $X$ é um espaço topológico $T_{0}$ e denso em si mesmo).

Seja $\alpha<|\mathcal{B}|=w(X)$ um ordinal. Após termos selecionado pontos distintos $p_{\beta}$ e $q_{\beta}$ pertencentes a $U_{\beta}$, para todo ordinal $\beta<\alpha$, escolheremos pontos distintos $p_{\alpha}, q_{\alpha} \in U_{\alpha}$ da seguinte maneira: denotemos por $A_{\alpha}$ o conjunto dos pontos de $U_{\beta}$ que foram designados para $A$, qualquer que seja $\beta<\alpha$. De maneira análoga, denotaremos por $B_{\alpha}$ o conjunto dos pontos de $U_{\beta}$ que foram designados para $B$, qualquer que seja $\beta<\alpha$. Escolheremos, portanto, dois pontos distintos $p_{\alpha}$ e $q_{\alpha}$ do conjunto $U_{\alpha} \backslash\left(A_{\alpha} \cup B_{\alpha}\right)$. Tal escolha somente não seria possível se $\left|U_{\alpha} \backslash\left(A_{\alpha} \cup B_{\alpha}\right)\right|<2$. Mostraremos, agora, que esta situação não ocorre. De fato, temos que $\left|U_{\alpha}\right| \geq \Delta(X) \geq \omega$ e, como $\alpha<|\mathcal{B}|=w(X)$, vale que $\left|A_{\alpha}\right|<w(X)$ e $\left|B_{\alpha}\right|<w(X)$. Logo,

$$
\left|A_{\alpha}\right|+\left|B_{\alpha}\right|<w(X)+w(X) \leq \Delta(X)+\Delta(X)=\Delta(X) \leq\left|U_{\alpha}\right|
$$

Como $\left|U_{\alpha}\right| \geq \omega$, segue que $\left|U_{\alpha} \backslash\left(A_{\alpha} \cup B_{\alpha}\right)\right| \geq \omega$.

É evidente que os conjuntos $A$ e $B$ assim construídos são ambos densos e disjuntos em $X$. Portanto, da proposição 2.1.3 segue que $X$ é um espaço topológico resolúvel.

Teorema 2.1.14. Se X é um espaço topológico tal que

$$
d(X)<\Delta(X)
$$

então $X$ é resolúvel.

Demonstração. Se $X$ não fosse um espaço topológico resolúvel então, pela proposição 2.1.3, concluiríamos que todo subconjunto denso de $X$ teria interior não vazio e, portanto, 
conteria algum subconjunto aberto não vazio de $X$. Logo, $\Delta(X) \leq|D|$, para todo subconjunto denso $D$ de $X$, o que implica $\Delta(X) \leq d(X)$, um absurdo.

Lema 2.1.15. Seja $X$ um espaço topológico $T_{0}$ e sem pontos isolados tal que todo aberto não vazio $U$ de $X$ contém um subconjunto aberto não vazio $H_{0}$ de $X$ verificando a seguinte propriedade:

$$
w\left(H_{0}\right) \leq\left|H_{0}\right|
$$

Então, $X$ é resolúvel.

Demonstração. Mostraremos que todo subconjunto aberto não vazio $U$ de $X$ contém um subconjunto não vazio $H_{0}$, resolúvel na topologia induzida. Do teorema 2.1.9 seguirá que $X$ é um espaço topológico resolúvel.

Seja $U$ um subconjunto aberto não vazio de $X$. Consideremos $U_{0}$ um subconjunto aberto não vazio de $U$, tal que $\left|U_{0}\right|=\Delta(U)$. O conjunto $U_{0}$ é, também, aberto em $X$ e, por hipótese, segue que $U_{0}$ contém um subconjunto aberto não vazio $H_{0}$ de $X$ tal que $w\left(H_{0}\right) \leq\left|H_{0}\right|$.

Sabemos que $\left|H_{0}\right|=\left|U_{0}\right|=\Delta(U)$. De fato, como $H_{0} \subset U_{0}$, temos que $\left|H_{0}\right| \leq\left|U_{0}\right|$. Contudo, $\left|U_{0}\right|=\Delta(U)$ e, como $H_{0}$ é, também, aberto em $U$, segue que $\Delta(U) \leq\left|H_{0}\right|$.

Como $X$ é um espaço topológico $T_{0}$ que não possui pontos isolados, segue que $U$ também o é e, portanto, $\Delta(U)$ é infinito. Logo, $\left|H_{0}\right| \geq \omega$.

Seja $V$ um aberto não vazio de $H_{0}$. Temos que $|V|=\left|H_{0}\right|$. De fato, como $V \subset H_{0} \subset U_{0}$, segue que $|V| \leq\left|U_{0}\right|$. Contudo, como $H_{0}$ é aberto em $X$, temos que $V$ também o é. Como $V \subset U$, segue que $V$ é aberto em $U$. Portanto, $|V| \geq \Delta(U)=\left|U_{0}\right|$. Portanto, $|V|=\left|U_{0}\right|=\left|H_{0}\right|$. Daí, segue que $\Delta\left(H_{0}\right)=\left|H_{0}\right|$. Logo, $w\left(H_{0}\right) \leq\left|H_{0}\right|=\Delta\left(H_{0}\right)$. 
Do teorema 2.1.13 segue que $H_{0}$, munido da topologia induzida, é um espaço resolúvel. Do teorema 2.1.9 decorre que $X$ é um espaço topológico resolúvel.

Apresentaremos, agora, os dois principais resultados desta seção. Eles nos permitirão concluir que muitos dos espaços topológicos sem pontos isolados com que usualmente trabalhamos são resolúveis.

Teorema 2.1.16. Todo espaço topológico $T_{2}$, localmente compacto e sem pontos isolados é resolúvel.

Demonstração. Seja $X$ um espaço topológico localmente compacto e $T_{2}$. Sabemos que todo subespaço aberto $U$ de $X$ é, também, localmente compacto e $T_{2}$. Portanto, $w(U) \leq|U|$. Do lema 2.1.15 segue que $X$ é resolúvel.

Teorema 2.1.17. Todo espaço topológico $T_{0}$, denso em si mesmo e que satisfaz o segundo axioma de enumerabilidade é resolúvel.

Demonstração. Do corolário 2.1.12 segue que se $X$ é um espaço topológico $T_{0}$ e denso em si mesmo, então $\Delta(X) \geq \omega$. Como, por hipótese, $X$ satisfaz o segundo axioma de enumerabilidade, temos que $w(X) \leq \omega$ e, portanto, $w(X) \leq \Delta(X)$. Do teorema 2.1.13 decorre que $X$ é resolúvel. 


\subsection{A noção de $\kappa$-resolubilidade}

Definição 2.2.1. Seja $\kappa>1$ um cardinal. Dizemos que um espaço topológico $X$ é $\kappa$ resolúvel se $X$ possui $\kappa$ subconjuntos densos, dois a dois disjuntos.

Observamos que um espaço topológico é resolúvel se, e somente se, é 2-resolúvel.

Fato 2.2.2. Sejam $\kappa>1$ um cardinal e $X$ um espaço topológico. Se X é $\kappa$-resolúvel, então $X$ é $\lambda$-resolúvel, para todo cardinal $1<\lambda<\kappa$.

Demonstração. Sejam $\kappa>1$ um cardinal infinito e $X$ um espaço topológico $\kappa$-resolúvel. Da definição 2.2.1 decorre que $X$ possui $\kappa$ subconjuntos densos, dois a dois disjuntos. Em particular, $X$ possui $\lambda$ subconjuntos densos, dois a dois disjuntos, para todo cardinal $1<\lambda<\kappa$. Daí, segue que $X$ é um espaço topológico $\lambda$-resolúvel.

Quando um subconjunto $Y$ de um espaço topológico $X$ for $\kappa$-resolúvel na topologia induzida, para algum cardinal $\kappa>1$, diremos que $Y$ é um subespaço $\kappa$-resolúvel de $X$.

Fato 2.2.3. Sejam $\kappa>1$ um cardinal e X um espaço topológico $\kappa$-resolúvel. Se $U$ é um subconjunto aberto de $X$, então $U$ é um subespaço $\kappa$-resolúvel de $X$.

Demonstração. Sejam $\kappa>1$ um cardinal e $X$ um espaço topológico $\kappa$-resolúvel. Na demonstração da proposição 2.1.7, mostramos que se $D$ é um subconjunto denso de $X$, então $D \cap U$ é um subconjunto denso de $U$, quando consideramos $U$ munido da topologia induzida.

Por hipótese, $X$ possui $\kappa$ subconjuntos densos, dois a dois disjuntos. Cada um deste subconjuntos de $X$, quando intersectados com $U$, será um subconjunto denso de 
$U$. Obviamente, quaisquer duas destas intersecções serão disjuntas. Portanto, $U$ é um subespaço $\kappa$-resolúvel de $X$.

Fato 2.2.4. Sejam $\kappa>1$ um cardinal e $X$ um espaço topológico. Se $Y$ é um subespaço $\kappa$-resolúvel de $X$, então $\bar{Y}$ é, também, um subespaço $\kappa$-resolúvel de $X$.

Demonstração. Consta, na demonstração da proposição 2.1.5, o fato de que se $X$ é um espaço topológico e $Y$ é um subespaço de $X$, então todo subconjunto denso de $Y$ é, também, denso em $\bar{Y}$.

Logo, se $Y$ é um subespaço $\kappa$-resolúvel de um espaço topológico $X$, existem $\kappa$ subconjuntos densos e dois a dois disjuntos de $Y$ que serão, em virtude da observação acima, densos em $\bar{Y}$. Portanto, $\bar{Y}$ também será um subespaço $\kappa$-resolúvel do espaço topológico $X$.

A demonstração do próximo resultado é análoga à da proposição 2.1.8.

Fato 2.2.5. Seja $\kappa>1$ um cardinal. Seja $X$ um espaço topológico tal que

$$
X=\bigcup_{i \in I} X_{i}
$$

onde $\left\{X_{i}\right\}_{i \in I}$ é uma família de subespaços $\kappa$-resolúveis de $X$. Então, $X$ é um espaço topológico $\kappa$-resolúvel.

Demonstração. Seja $\mathcal{M}$ uma família maximal de subespaços $\kappa$-resolúveis de $X$, dois a dois disjuntos. Afirmamos que $\bigcup \mathcal{M}$ é um subconjunto denso de $X$. Com efeito, se isto não ocorresse,

$$
U: = X \backslash \longdiv { \bigcup \mathcal { M } }
$$


seria um subconjunto aberto não vazio de $X$. Como

$$
X=\bigcup_{i \in I} X_{i}
$$

existirá $i \in I$ tal que $U \cap X_{i} \neq \emptyset$. Do fato 2.2.3 decorre que $U \cap X_{i}$ é um subespaço $\kappa$-resolúvel de $X_{i}$ e, portanto, $U \cap X_{i}$ é um subespaço $\kappa$-resolúvel de $X$.

Logo, $\mathcal{M} \cup\left\{U \cap X_{i}\right\}$ é uma família de subespaços $\kappa$-resolúveis de $X$, dois a dois disjuntos, que contém $\mathcal{M}$ propriamente - o que é absurdo, em vista da maximalidade de $\mathcal{M}$. Portanto, $\bigcup \mathcal{M}$ é um subconjunto denso de $X$.

Para cada $Y \in \mathcal{M}$, tomemos $\left\{D_{j}^{Y}: j<\kappa\right\}$ uma família de subconjuntos densos de $Y$, dois a dois disjuntos. Para cada $j<\kappa$, consideremos

$$
D_{j}:=\bigcup_{Y \in \mathcal{M}} D_{j}^{Y} .
$$

Afirmamos que $\left\{D_{j}: j<\kappa\right\}$ é uma família de subconjuntos densos de $X$, dois a dois disjuntos.

De fato, $\operatorname{sejam} i, j<\kappa, i \neq j$. Se houvesse

$$
p \in D_{i} \cap D_{j}=\left(\bigcup_{Y \in \mathcal{M}} D_{i}^{Y}\right) \cap\left(\bigcup_{Y \in \mathcal{M}} D_{j}^{Y}\right)
$$

existiriam $Y_{1}, Y_{2} \in \mathcal{M}$ tais que

$$
p \in D_{i}^{Y_{1}} \cap D_{j}^{Y_{2}} \subset Y_{1} \cap Y_{2}
$$

o que é um absurdo, já que os elementos de $\mathcal{M}$ são dois a dois disjuntos. Portanto, $\left\{D_{j}: j<\kappa\right\}$ é uma família de subconjuntos de $X$, dois a dois disjuntos.

Resta mostrar que, para cada $i<\kappa, D_{i}$ é um subconjunto denso de $X$. Para tanto, consideremos $U$ um subconjunto aberto não vazio de $X$. Como $\bigcup \mathcal{M}$ é densa em $X$, temos que $U \cap(\bigcup \mathcal{M}) \neq \emptyset$. Portanto, existe $Y \in \mathcal{M}$ tal que $U \cap Y \neq \emptyset$. Logo, $U \cap Y$ é um 
subconjunto aberto não vazio de $Y$ e, portanto, $D_{i}^{Y} \cap(U \cap Y) \neq \emptyset$, já que $D_{i}^{Y}$ é denso em $Y$. Em particular, segue que $D_{i}^{Y} \cap U \neq \emptyset$ e, portanto, $D_{i} \cap U \neq \emptyset$. Logo, $D_{i}$ é denso em $X$ e, portanto, $\left\{D_{j}: j<\kappa\right\}$ é uma família de subconjuntos densos de $X$.

Logo, $X$ é $\kappa$-resolúvel.

Seja $\kappa>1$ um cardinal. Sabemos que se $X$ é um espaço topológico $\kappa$-resolúvel então, para todo aberto não vazio $U$ de $X$ vale que $\kappa \leq|U|$ e, portanto, $\kappa \leq \Delta(X)$. Esta observação justifica a terminologia utilizada na próxima definição.

Definição 2.2.6. Um espaço topológico $X$ tal que $\Delta(X)>1$ é dito maximalmente resolúvel se $X$ for $\Delta(X)$-resolúvel.

Há espaços topológicos resolúveis que não são maximalmente resolúveis. Apresentamos, no quarto capítulo desta dissertação, a construção de espaço topológico regular que é $n$ resolúvel, mas que não é $(n+1)$-resolúvel, para um dado número natural $n>1$. Como o caráter de dispersão deste espaço é infinito, em virtude do corolário 2.1.12, o mesmo não será maximalmente resolúvel, pelo fato 2.2.2.

O restante desta seção será dedicado à generalização dos principais teoremas apresentados na seção anterior.

Definição 2.2.7. Uma família $\mathcal{S}$ de subconjuntos de um espaço topológico $X$ é dita um $\pi$-network se

(1) $S \neq \emptyset$, para todo $S \in \mathcal{S}$;

(2) Todo subconjunto aberto não vazio de $X$ contém algum elemento de $\mathcal{S}$. 
Teorema 2.2.8. Seja $(X, \tau)$ um espaço topológico sem pontos isolados tal que $w(X)<\omega$. Então, X é maximalmente resolúvel.

Demonstração. Seja $\mathcal{B}=\left\{U_{1}, \ldots, U_{n}\right\}$ uma base de abertos de $X$ tal que $|\mathcal{B}|=w(X)$. Consideremos

$$
\mathcal{S}=\{S \in \tau \backslash\{\emptyset\}: S \text { contém propriamente nenhum aberto não vazio de } X\}
$$

Afirmamos que $\mathcal{S}$ é um $\pi$-network. De fato, da definição de $\mathcal{S}$, segue que $S \neq \emptyset$, para todo $S \in \mathcal{S}$. Portanto, a fim de provar que $\mathcal{S}$ é um $\pi$-network de $X$, basta assegurar que todo subconjunto aberto não vazio de $X$ contém um elemento de $\mathcal{S}$. Seja $U$ um subconjunto aberto não vazio de $X$. Podemos supor (reordenando $\mathcal{B}$, se necessário), que $U \supset U_{1}$. Se $U_{1} \in \mathcal{S}$, nada temos a fazer. Se não, $U_{1}$ contém propriamente um subconjunto aberto não vazio $V_{1}$ de $X$. Este, por sua vez, contém um elemento de $\mathcal{B} \backslash\left\{U_{1}\right\}$, que podemos supor ser $U_{2}$. Repetimos o mesmo argumento para $U_{2}$. Afirmamos que se $U_{1}, \ldots, U_{n-1} \notin \mathcal{S}$, então $U_{n} \in \mathcal{S}$. Com efeito, temos que $U \supsetneqq U_{1} \supsetneqq U_{2} \supsetneqq \ldots \supsetneqq U_{n-1} \supsetneqq U_{n}$. Se $U_{n} \notin \mathcal{S}$, encontraremos um subconjunto aberto não vazio $V_{n}$ de $X$ tal que $U_{n} \supsetneqq V_{n}$. Como $\mathcal{B}$ é uma base de $X$, deveria existir $B \in \mathcal{B}$ tal que $B \subset V_{n}$, o que não ocorre, já que $\mathcal{B}=\left\{U_{1}, \ldots, U_{n}\right\}$ e $U_{i} \supsetneqq V_{n}$, para todo $i \in\{1, \ldots, n\}$. Logo, $U_{n} \in \mathcal{S}$. Portanto, $\mathcal{S}$ é um $\pi$-network.

Para cada $S \in \mathcal{S}$, consideremos uma função injetora

$$
f_{S}: \Delta(X) \rightarrow S
$$

Note que uma tal função existe, pois todo elemento $S$ de $\mathcal{S}$ é um subconjunto aberto não vazio de $X$ e, portanto, $\Delta(X) \leq|S|$. Afirmamos que, para cada $\eta \in \Delta(X)$, o conjunto

$$
D_{\eta}:=\left\{f_{S}(\eta): S \in \mathcal{S}\right\}
$$

é denso em $X$ e, além disso, se $\alpha, \beta \in \Delta(X)$ e $\alpha \neq \beta$, então $D_{\alpha} \cap D_{\beta}=\emptyset$. 
Com efeito, fixemos $\eta \in \Delta(X)$ e consideremos $U$ um subconjunto aberto não vazio qualquer de $X$. Suponhamos, por absurdo, que $U \cap D_{\eta}=\emptyset$. Então, $f_{S}(\eta) \notin U$, para todo $S \in \mathcal{S}$. Como $f_{S}(\eta) \in S$, para todo $S \in \mathcal{S}$, temos que $S \not \subset U$, para todo $S \in \mathcal{S}$, o que contradiz o fato de $\mathcal{S}$ ser um $\pi$-network. Logo, $D_{\eta}$ é denso em $X$.

Além disso, se existissem $\alpha, \beta \in \Delta(X), \alpha \neq \beta$, tais que $D_{\alpha} \cap D_{\beta} \neq \emptyset$, encontraríamos $S_{1}, S_{2} \in \mathcal{S}$ tais que $f_{S_{1}}(\alpha)=f_{S_{2}}(\beta)$ e $S_{1} \neq S_{2}$ (pois $f_{S}$ é injetora, para todo $S \in \mathcal{S}$ ). Portanto, $S_{1} \cap S_{2} \neq \emptyset$. Como $S_{1} \cap S_{2} \subset S_{1}, S_{1} \cap S_{2} \subset S_{2}$ e $S_{1} \cap S_{2} \in \tau \backslash\{\emptyset\}$, teremos que $S_{1}$ e $S_{2}$ contêm, propriamente, um aberto não vazio de $X$, o que é absurdo, já que $S_{1}, S_{2} \in \mathcal{S}$. Portanto, se $\alpha, \beta \in \Delta(X)$ e $\alpha \neq \beta$, então $D_{\alpha} \cap D_{\beta}=\emptyset$.

Logo, $X$ é maximalmente resolúvel.

Lema (do refinamento disjunto) 2.2.9. Sejam $\kappa$ um cardinal infinito e $\mathcal{S}$ uma coleção de conjuntos tais que $|\mathcal{S}|=\kappa$ e $|S|=\kappa$, para todo $S \in \mathcal{S}$. Existe uma família $\{T(S): S \in \mathcal{S}\}$ de conjuntos dois a dois disjuntos tais que

(1) $T(S) \subset S$, para todo $S \in \mathcal{S}$;

(2) $|T(S)|=\kappa$, para todo $S \in \mathcal{S}$.

Demonstração. Vamos indexar a coleção $\mathcal{S}$ por

$$
\mathcal{S}=\left\{S_{\alpha}\right\}_{\alpha<\kappa}
$$

e escrever cada elemento $S_{\alpha} \in \mathcal{S}$ como

$$
S_{\alpha}=\bigcup_{\beta<\kappa} S_{\alpha, \beta}
$$


onde $\left|S_{\alpha, \beta}\right|=\kappa$, para todos $\alpha, \beta<\kappa$. Fixemos $b_{0,0} \in S_{0,0}$.

Como o conjunto $\kappa \times \kappa$ é equipotente a $\kappa$, existe uma função bijetora

$$
\varphi: \kappa \times \kappa \rightarrow \kappa
$$

$\operatorname{com} \varphi(0,0)=0$.

Ordenemos $\kappa \times \kappa$ da seguinte maneira:

$$
(\gamma, \delta) \ll(\alpha, \beta) \text { se, e somente se } \varphi(\gamma, \delta)<\varphi(\alpha, \beta)
$$

onde $<$ denota a ordem usual de $\kappa$.

Consideremos $(\alpha, \beta) \in \kappa \times \kappa$. O conjunto dos pontos $b_{\gamma, \delta} \in S_{\gamma, \delta}$ tais que $(\gamma, \delta) \ll(\alpha, \beta)$ tem cardinalidade menor do que $\kappa$, pois $(\gamma, \delta) \ll(\alpha, \beta)$ implica $\varphi(\gamma, \delta)<\varphi(\alpha, \beta)<\kappa$. Logo,

$$
S_{\alpha, \beta} \backslash\left\{b_{\gamma, \delta} \in S_{\gamma, \delta}:(\gamma, \delta) \ll(\alpha, \beta)\right\}
$$

é não vazio e, portanto, podemos escolher

$$
b_{\alpha, \beta} \in S_{\alpha, \beta} \backslash\left\{b_{\gamma, \delta} \in S_{\gamma, \delta}:(\gamma, \delta) \ll(\alpha, \beta)\right\}
$$

Para cada $\alpha<\kappa$, seja

$$
T_{\alpha}=\left\{b_{\alpha, \beta}: \beta<\kappa\right\} \subset S_{\alpha}
$$

Como os elementos de $T_{\alpha}$ são dois a dois distintos, para todo $\alpha<\kappa$, concluímos que $\left|T_{\alpha}\right|=\kappa$, para todo $\alpha<\kappa$.

Além disso, temos que os conjuntos $T_{\alpha}$ acima definidos são dois a dois disjuntos.

Teorema 2.2.10. Seja $X$ um espaço topológico $T_{0}$ e denso em si mesmo. Se $X$ possui um $\pi$-network $\mathcal{S}$ tal que $|\mathcal{S}| \leq \Delta(X)$ e cada $S \in \mathcal{S}$ verifica $|S| \geq \Delta(X)$, então $X$ é maximalmente resolúvel. 
Demonstração. Definamos

$$
\mathcal{S}^{*}=\mathcal{S} \backslash\{X\}
$$

Como $\mathcal{S}$ é, por hipótese, um $\pi$-network, segue que $\mathcal{S}^{*}$ também o é. Além disso, temos que $|S| \geq \Delta(X)$, para todo $S \in \mathcal{S}^{*}$.

Para cada $S \in \mathcal{S}^{*}$, escolhamos $\Delta(X)$ pontos de $S$ e denotemos por $S^{\prime}$ o conjunto que tem como elementos exatamente estes pontos. Seja

$$
\mathcal{S}^{\prime}=\left\{S^{\prime}: S \in \mathcal{S}^{*}\right\}
$$

Como $\left|\mathcal{S}^{\prime}\right| \leq\left|\mathcal{S}^{*}\right| \leq|\mathcal{S}|$, temos que $\left|\mathcal{S}^{\prime}\right| \leq \Delta(X)$. Notemos que todos os elementos de $\mathcal{S}^{\prime}$ têm cardinalidade $\Delta(X)$.

Seja $\left\{S_{i}^{\prime}\right\}_{i<\mu}$ uma indexação de $\mathcal{S}^{\prime}$, onde $\mu \leq \Delta(X)$.

Se $\mu=\Delta(X)$, fazemos $\mathcal{S}^{\prime \prime}=\mathcal{S}^{\prime}$. Podemos aplicar o lema do refinamento disjunto para $\mathcal{S}^{\prime \prime}$, obtendo uma família $\left\{T(S): S \in \mathcal{S}^{\prime \prime}\right\}$ que é, ainda, um $\pi$-network de $X$.

Suponhamos que $\mu<\Delta(X)$. Como

$$
|\{X \backslash\{x\}: x \in X\}|=|X| \geq \Delta(X)>\mu
$$

existe $x \in X$ tal que $X \backslash\{x\} \neq S_{i}^{\prime}$, para todo $i<\mu$. Para cada $i<\mu$, existe $x_{i} \in X \backslash\{x\}$ tal que $x_{i} \in X \backslash\{x\}$ e $x_{i} \notin S_{i}^{\prime}$. De fato, se isto não ocorresse, teríamos que $X \backslash\{x\} \subset S_{i}^{\prime}$ e, portanto, $S_{i}^{\prime}=X \backslash\{x\}$ ou $S_{i}^{\prime}=X$, o que não acontece.

Escolhamos $\Delta(X)$ pontos de $X$ e consideremos o conjunto formado por estes elementos. A esta coleção acrescentemos, para todo $i<\mu$, o ponto $x_{i}$ e denotemos por $S_{x}$ o conjunto resultante.

Temos que $\left|S_{x}\right|=\Delta(X)$ e $S_{x} \neq S_{i}^{\prime}$, para todo $i<\mu$. Se $S_{x}=X$, retiro um ponto $y$ de $S_{x}$, com $y \neq x_{i}$, para todo $i<\mu$.

Repetimos este processo (agora, para $\left\{S_{i}^{\prime}\right\}_{i<\mu} \cup\left\{S_{x}\right\}$ ) até obter uma coleção $\mathcal{S}^{\prime \prime}$ de $\Delta(X)$ conjuntos, que constitui um $\pi$-network para $X$, cada um de cardinalidade $\Delta(X)$. Para tal 
coleção, aplico o lema do refinamento disjunto, obtendo uma família $\left\{T(S): S \in \mathcal{S}^{\prime \prime}\right\}$ que é, ainda, um $\pi$-network de $X$.

Para cada $S \in \mathcal{S}^{\prime \prime}$, seja $f_{S}: \Delta(X) \rightarrow T(S)$ injetora. Tal função existe, pois $|T(S)|=\Delta(X)$. Para cada $\eta \in \Delta(X)$, seja

$$
D_{\eta}=\left\{f_{S}(\eta): S \in \mathcal{S}^{\prime \prime}\right\}
$$

Afirmamos que $D_{\alpha} \cap D_{\beta}=\emptyset$, se $\alpha, \beta \in \Delta(X)$ e $\alpha \neq \beta$. De fato, se existissem $\alpha, \beta \in \Delta(X)$ tais que $\alpha \neq \beta$ e $D_{\alpha} \cap D_{\beta} \neq \emptyset$, existiriam $S_{1}, S_{2} \in \mathcal{S}^{\prime \prime}$ tais que $f_{S_{1}}(\alpha)=f_{S_{2}}(\beta)$. Como $f_{S}$ é injetora, para todo $S \in \mathcal{S}^{\prime \prime}$, segue que $S_{1} \neq S_{2}$. Mas, neste caso, $T\left(S_{1}\right) \cap T\left(S_{2}\right) \neq \emptyset$, o que é um absurdo.

Afirmamos, ainda, que cada $D_{\eta}$ é denso em $X$, para todo $\eta \in \Delta(X)$. De fato, seja $U$ um subconjunto aberto não vazio de $X$. Se $U \cap D_{\eta}=\emptyset$, então $f_{S}(\eta) \notin U$, para todo $S \in \mathcal{S}^{\prime \prime}$. Logo, $T(S) \not \subset U$, para todo $S \in \mathcal{S}^{\prime \prime}$, o que é absurdo, pois $\left\{T(S): S \in \mathcal{S}^{\prime \prime}\right\}$ é um $\pi$-network de $X$.

Logo, $X$ é maximalmente resolúvel.

Corolário 2.2.11. Se $X$ é um espaço topológico $T_{0}$, denso em si mesmo, com $w(X)=\omega$, então X é maximalmente resolúvel.

Demonstração. Como $X$ é um espaço topológico $T_{0}$ e denso em si mesmo, segue que $\Delta(X) \geq \omega$. Seja $\mathcal{B}$ uma base de abertos de $X$ tal que $|\mathcal{B}|=w(X)$. Temos, em particular, que $\mathcal{B}$ é um $\pi$-network. Além disso, é claro que todo elemento de $\mathcal{B}$ tem cardinalidade maior ou igual a $\Delta(X)$. Do teorema 2.2.10 segue que $X$ é maximalmente resolúvel. 
Do teorema 2.2.8 e do corolário 2.2.11 segue que todo espaço topológico $T_{0}$, denso em si mesmo e que satisfaz o segundo axioma de enumerabilidade é maximalmente resolúvel.

Teorema 2.2.12. Se $(X, \tau)$ é um espaço topológico metrizável e sem pontos isolados, então $X$ é maximalmente resolúvel.

Demonstração. Seja

$$
\mathcal{U}=\{U \in \tau \backslash\{\emptyset\}:|U|=|V| \text {, para todo aberto não vazio } V \text { de } U\}
$$

Afirmamos que $\bigcup \mathcal{U}$ é um subconjunto denso de $X$. De fato, seja $\Omega$ um aberto não vazio de $X$. Mostraremos que $\Omega$ contém um elemento de $\mathcal{U}$ e, portanto, que $\Omega \cap \bigcup \mathcal{U} \neq \emptyset$. Seja $W$ um aberto de $X$ tal que $|\Omega \cap W|=\Delta(\Omega)$. Temos que $\Omega \cap W \in \mathcal{U}$ pois, do contrário, existiria $V$ um subconjunto aberto de $X$ tal que $V \cap \Omega \cap W \neq \emptyset$ e $|V \cap \Omega \cap W|<|\Omega \cap W|$, ou seja, tal que $|V \cap \Omega \cap W|<\Delta(\Omega)$, o que é absurdo, uma vez que $V \cap \Omega \cap W$ é, também, aberto em $\Omega$.

Se mostrarmos que cada $U \in \mathcal{U}$ é $\Delta(X)$-resolúvel, do fato 2.2 .5 seguirá que $\bigcup \mathcal{U}$ é $\Delta(X)$-resolúvel e, com o auxílio do fato 2.2.4, concluiremos que $X=\overline{\bigcup \mathcal{U}}$ é maximalmente resolúvel.

Notemos, primeiramente, que $\Delta(X) \leq \Delta(U)$, para todo subconjunto aberto não vazio $U$ de $X$. De fato, como todo aberto em $U$ é, também, aberto em $X$, segue que $\Delta(X) \leq|V|$, para todo aberto não vazio $V$ de $U$. Logo, $\Delta(X) \leq \Delta(U)$.

Mostremos, portanto, que cada $U \in \mathcal{U}$ é $\Delta(U)$-resolúvel. Do fato 2.2 .2 seguirá que $U$ é $\Delta(X)$-resolúvel.

Seja $U \in \mathcal{U}$. Seja $\mathcal{S}$ uma base de abertos de $U$ com $|\mathcal{S}|=w(U)$. É evidente que $\mathcal{S}$ é um $\pi$-network. Para todo $S \in \mathcal{S}$ vale que $|S| \geq \Delta(U)$. Como $X$ é, por hipótese, metrizável, segue que $U$ também o é e, portanto, $w(U)=d(U)$. Logo

$$
|\mathcal{S}|=w(U)=d(U) \leq|U|=\Delta(U)
$$


Do teorema 2.2.10 decorre que $U$ é $\Delta(U)$-resolúvel.

O próximo resultado generaliza o teorema 2.1.16.

Teorema 2.2.13. Se $X$ é um espaço topológico localmente compacto, $T_{2}$ e sem pontos isolados, então X é maximalmente resolúvel.

Demonstração. Seja $\mathcal{U}$ a coleção dos subconjuntos abertos não vazios $U$ de $X$ tais que $\bar{U}$ é compacto, $|\bar{U}|=|U|$ e $|U|=|V|$, para todo aberto não vazio $V$ de $U$.

Afirmamos que $\bigcup \mathcal{U}$ é um subconjunto denso em $X$. De fato, seja $\Omega$ um aberto não vazio de $X$. Mostremos que $\Omega$ contém um elemento de $\mathcal{U}$ e, portanto, que $\Omega \cap \bigcup \mathcal{U} \neq \emptyset$.

Seja $V$ um aberto de $X$ tal que $|\Omega \cap V|=\Delta(\Omega)$. Seja $x \in \Omega \cap V$. Como $X$ é localmente compacto, existe uma vizinhança compacta $W$ de $x$ em $X$ tal que $x \in W \subset \Omega \cap V$. Do fato de $X$ ser um espaço topológico $T_{2}$, segue $W=\bar{W}$. Como $W$ é uma vizinhança de $x$, temos que $\operatorname{int}(W) \neq \emptyset$.

Afirmamos que $\operatorname{int}(W) \in \mathcal{U}$.

Com efeito, $\operatorname{int}(W) \subset W$ e, portanto, $\overline{\operatorname{int}(W)} \subset \bar{W}=W$. Como $W$ é compacto e $\overline{\operatorname{int}(W)}$ é fechado, segue que $\overline{\operatorname{int}(W)}$ é compacto.

Temos, também, que $|\operatorname{int}(W)|=|\overline{\operatorname{int}(W)}|$. De fato, $\operatorname{int}(W)$ é aberto em $X$ e como $\operatorname{int}(W) \subset \Omega$, temos que $\operatorname{int}(W)$ é aberto em $\Omega$. Portanto, $|\Omega \cap V| \leq|\operatorname{int}(W)|$. Como $\operatorname{int}(W) \subset \Omega \cap V$, temos que $|\operatorname{int}(W)| \leq|\Omega \cap V|$. Logo, $|\operatorname{int}(W)|=|\Omega \cap V|$. Como $\overline{\operatorname{int}(W)} \subset \bar{W} \subset \Omega \cap V$, concluímos que $|\operatorname{int}(W)|=|\overline{\operatorname{int}(W)}|$.

Resta mostrar que $|\operatorname{int}(W)|=|A|$, para todo aberto não vazio $A \operatorname{de} \operatorname{int}(W)$. Caso contrário, existiria $A$ um subconjunto aberto não vazio $\operatorname{de} \operatorname{int}(W)$ tal que $|A|<|\operatorname{int}(\mathrm{W})|$. Como $A$ é, em particular, aberto em $X$ e $\operatorname{int}(W) \subset \Omega$, temos que $A$ é aberto em $\Omega$. Logo

$$
|A|<|\operatorname{int}(W)| \leq|\Omega \cap V|
$$


o que contraria a minimalidade de $\Omega \cap V$.

Logo, $\operatorname{int}(W) \in \mathcal{U}$ e $\operatorname{int}(W) \subset \Omega$.

Se mostrarmos que cada $U \in \mathcal{U}$ é $\Delta(X)$-resolúvel, do fato 2.2.5 seguirá que $\bigcup \mathcal{U}$ é $\Delta(X)$-resolúvel e, com o auxílio do fato 2.2.4, concluiremos que $X=\overline{\bigcup \mathcal{U}}$ é maximalmente resolúvel.

Notemos, primeiramente, que $\Delta(X) \leq \Delta(U)$, para todo subconjunto aberto não vazio $U$ de $X$. De fato, como todo aberto em $U$ é, também, aberto em $X$, segue que $\Delta(X) \leq|V|$, para todo aberto não vazio $V$ de $U$. Logo, $\Delta(X) \leq \Delta(U)$.

Mostremos, portanto, que cada $U \in \mathcal{U}$ é $\Delta(U)$-resolúvel. Do fato 2.2 .2 seguirá que $U$ é $\Delta(X)$-resolúvel.

Seja $U \in \mathcal{U}$. Seja $\mathcal{S}$ uma base de abertos de $U \operatorname{com}|\mathcal{S}|=w(U)$. Temos que todo $S \in \mathcal{S}$ satisfaz $|S| \geq \Delta(U)$. Como $\bar{U}$ é compacto e $T_{2}$ decorre que $|w(\bar{U})| \leq|\bar{U}|$. Logo

$$
|\mathcal{S}|=w(U) \leq w(\bar{U}) \leq|\bar{U}|=|U|=\Delta(U)
$$

Do teorema 2.2.10 segue que $U$ é $\Delta(U)$-resolúvel. 


\section{Capítulo 3}

\section{Espaços topológicos irresolúveis}

A primeira seção deste capítulo será dedicada aos diversos graus de irresolubilidade que um espaço topológico pode apresentar. Mostraremos que todo espaço topológico maximal e $T_{1}$ é, também, submaximal. Provaremos, ainda, que todo espaço topológico submaximal é, em particular, hereditariamente irresolúvel. Evidentemente, todo espaço topológico hereditariamente irresolúvel é hereditariamente irresolúvel por abertos e estes últimos são, por sua vez, irresolúveis. Demonstraremos, por fim, que a recíproca de algumas destas afirmações não são válidas. Os contra-exemplos utilizados com este propósito foram extraídos de [14]. Na seção seguinte, desenvolveremos a técnica de expansão de espaços topológicos - introduzida em [8] - a qual nos permitirá construir um exemplo de espaço topológico completamente regular, hereditariamente irresolúvel e denso em si mesmo. 


\subsection{Graus de irresolubilidade}

Um espaço topológico é dito irresolúvel se o mesmo não possui dois subconjuntos densos complementares. De acordo com a proposição 2.1.3, um espaço topológico $X$ é irresolúvel se, e somente se, todo subconjunto denso de $X$ tem interior não vazio.

Definição 3.1.1. Dizemos que um espaço topológico $X$ é hereditariamente irresolúvel por abertos - abreviadamente, $O H I$ - se todo subespaço aberto não vazio de $X$ for irresolúvel.

Evidentemente, todo espaço topológico hereditariamente irresolúvel por abertos é irresolúvel.

O próximo resultado fornece uma caracterização interessante dos espaços topológicos hereditariamente irresolúveis por abertos, a qual será fortemente utilizada no quinto capítulo desta dissertação.

Teorema 3.1.2. Um espaço topológico X é OHI se, e somente se, todo subconjunto denso de $X$ tem interior denso em $X$.

Demonstração. Sejam $X$ um espaço topológico OHI e $D$ um subconjunto denso de $X$. Mostraremos que $D$ tem interior denso em $X$. Suponhamos, por absurdo, que int $(D)$ não seja denso em $X$. Neste caso, teremos que $X \backslash \overline{\operatorname{int}(D)}$ é um subconjunto aberto não vazio de $X$. Além disso, todo aberto não vazio de $X$ contido em $X \backslash \overline{\operatorname{int}(D)}$ intersecta, necessariamente, o conjunto $X \backslash D$. De fato, se $V$ é um aberto não vazio de $X$ contido em $X \backslash \overline{\operatorname{int}(D)}$, então $V \cap \overline{\operatorname{int}(D)}=\emptyset \mathrm{e}$, portanto, $V \cap \operatorname{int}(D)=\emptyset$. Daí, segue que $V \subset X \backslash \operatorname{int}(D) \subset \overline{(X \backslash D)}$ e, portanto, $V \cap(X \backslash D) \neq \emptyset$. Contudo, uma vez que $D$ é denso em $X$, todo aberto de $X$ contido em $X \backslash \overline{\operatorname{int}(D)}$ intersecta, também, $D$. Desta forma, concluímos que $X \backslash \overline{\operatorname{int}(D)}$ é resolúvel na topologia induzida, o que é absurdo, uma vez que $X$ é OHI. Logo, $\operatorname{int}(D)$ é denso em $X$. 
Reciprocamente, seja $X$ um espaço topológico tal que todo subconjunto denso de $X$ tenha interior denso em $X$. Suponhamos, por absurdo, que $X$ não seja OHI. Então, existe um subconjunto aberto não vazio de $X$, o qual denotaremos por $U$, que é resolúvel na topologia induzida. Temos, portanto, que $U=D_{1} \cup D_{2}$, onde $D_{1}$ e $D_{2}$ são subconjuntos densos e disjuntos de $U$. Afirmamos que o conjunto $X \backslash D_{1}$ é denso em $X$. Com efeito, seja $V$ um aberto não vazio de $X$, qualquer. Se $V \cap U \neq \emptyset$, então $D_{2} \cap(U \cap V) \neq \emptyset$ e, portanto, $D_{2} \cap V \neq \emptyset$. Como $D_{2} \subset X \backslash D_{1}$, concluímos que $\left(X \backslash D_{1}\right) \cap V \neq \emptyset$. Se $V \cap U=\emptyset$, então $V \subset X \backslash U$. Como $D_{1} \subset U$, temos que $(X \backslash U) \subset\left(X \backslash D_{1}\right)$ e, portanto, $V \subset\left(X \backslash D_{1}\right)$. Logo, $\left(X \backslash D_{1}\right) \cap V \neq \emptyset$. Por hipótese, o interior de $\left(X \backslash D_{1}\right)$ é denso em $X$ e, portanto, tal conjunto intersecta $U$. Como $\emptyset \neq\left[U \cap \operatorname{int}\left(X \backslash D_{1}\right)\right] \subset U \backslash D_{1}$, temos que $D_{1} \cap\left[U \cap \operatorname{int}\left(X \backslash D_{1}\right)\right]=\emptyset$, o que é absurdo, já que $D_{1}$ é denso em $U$.

Definição 3.1.3. Dizemos que um espaço topológico $X$ é hereditariamente irresolúvel abreviadamente, $H I$ - se todo subespaço não vazio de $X$ for irresolúvel.

Observamos que todo espaço topológico hereditariamente irresolúvel é, em particular, hereditariamente irresolúvel por abertos.

Teorema (da decomposição de Hewitt) 3.1.4. Todo espaço topológico $X$ se escreve como união de dois subconjuntos disjuntos $A$ e $B$, onde $A$ é um fechado resolúvel na topologia induzida e $B$ é um aberto HI na topologia induzida. Além disso, X é resolúvel se, e somente se $B=\emptyset$ e $X$ é $H I$ se, e somente se, $A=\emptyset$.

Demonstração. Seja $X$ um espaço topológico e seja $\mathcal{R}$ a família de todos os subespaços resolúveis de $X$, ordenada pela inclusão. Seja $\mathcal{C}$ uma cadeia em $\mathcal{R}$. Da proposição 2.1.8 segue que $\bigcup \mathcal{C}$ é um subespaço resolúvel de $X$ e, portanto, é um majorante de $\mathcal{C}$. Do lema 
de Kuratowski-Zorn segue que $\mathcal{R}$ possui um elemento maximal $A$. Como $A$ é resolúvel, $\bar{A}$ também o é, e em virtude da maximalidade de $A$, segue que $A=\bar{A}$, ou seja, $A$ é fechado em $X$. Tomemos $B:=X \backslash A$. Temos que $B$ é um subconjunto aberto de $X$, HI. De fato, se existisse $S \subset B \subset X$ resolúvel na topologia induzida, então $A \cup S$ seria, também, resolúvel, contradizendo a maximalidade de $A$. Logo, $B$ é HI.

Em virtude do teorema 2.1.9 segue que $X$ é resolúvel se, e só se, $B=\emptyset$. A última asserção decorre diretamente da definição de irresolubilidade hereditária.

Definição 3.1.5. Dizemos que um espaço topológico $X$ é submaximal se todo subconjunto denso de $X$ for aberto em $X$.

É imediato que todo espaço topológico submaximal é irresolúvel. De fato, se $X$ é um espaço topológico não vazio e submaximal, então quaisquer dois subconjuntos densos de $X$ se intersectam, uma vez que ambos são abertos não vazios de $X$. O próximo teorema afirma que todo espaço topológico submaximal é, na verdade, HI.

Teorema 3.1.6. Se X é um espaço topológico submaximal, então X é HI.

Demonstração. Seja $X$ um espaço topológico submaximal e suponhamos, por absurdo, que $X$ não seja hereditariamente irresolúvel. Existe, portanto, um subconjunto $Y$ de $X$ resolúvel na topologia induzida. Sejam $D_{1}$ e $D_{2}$ subconjuntos complementares de $Y$, ambos densos em $Y$.

Afirmamos que o conjunto $D_{1} \cup(X \backslash \bar{Y})$ é denso em $X$. Com efeito, seja $U$ um aberto não vazio de $X$. Se $U \cap Y \neq \emptyset$, temos que $D_{1} \cap(U \cap Y) \neq \emptyset$, uma vez que $U \cap Y$ é aberto em $Y$ e $D_{1}$ é um subconjunto denso de $Y$. Em particular, temos que $D_{1} \cap U \neq \emptyset$ e, portanto, $\left[D_{1} \cup(X \backslash \bar{Y})\right] \cap U \neq \emptyset$. Se $U \cap Y=\emptyset$, segue que $U \cap \bar{Y}=\emptyset$, já que $U$ é aberto em $X$. Logo, 
$U \subset X \backslash \bar{Y}$ e, portanto, $\left[D_{1} \cup(X \backslash \bar{Y})\right] \cap U \neq \emptyset$. Logo, $D_{1} \cup(X \backslash \bar{Y})$ é um subconjunto denso em $X$.

Mostremos, contudo, que o conjunto em questão não é aberto em $X$. De fato, se $D_{1} \cup(X \backslash \bar{Y})$ fosse aberto em $X$ teríamos, em particular, que para todo $p \in D_{1}$, existiria uma vizinhança aberta de $p$ em $X$, a qual será denotada por $U_{p}$, tal que $p \in U_{p} \subset D_{1} \cup(X \backslash \bar{Y})$. Logo, $U_{p} \cap Y$ é, por definição, uma vizinhança aberta de $p$ em $Y$. Portanto,

$$
U_{p} \cap Y=U_{p} \cap\left(D_{1} \cup D_{2}\right) \subset\left[D_{1} \cup(X \backslash \bar{Y})\right] \cap\left(D_{1} \cup D_{2}\right)=D_{1} .
$$

Como $D_{1}$ e $D_{2}$ são subconjuntos disjuntos de $Y$, temos que $D_{2} \cap(U \cap Y)=\emptyset$, o que contradiz o fato de $D_{2}$ ser um subconjunto denso de $Y$.

Logo, $D_{1} \cup(X \backslash \bar{Y})$ não é aberto em $X$, o que é absurdo, uma vez que $X$ é um espaço topológico submaximal. Portanto, $X$ é hereditariamente irresolúvel.

Definição 3.1.7. Um espaço topológico $(X, \tau)$ é dito maximal se é denso em si mesmo e toda topologia $\tau^{\prime}$ sobre o conjunto $X$ estritamente mais fina do que $\tau$ faz com que o espaço topológico $\left(X, \tau^{\prime}\right)$ possua pontos isolados.

Teorema 3.1.8. Seja $(X, \tau)$ um espaço topológico $T_{1}$. Se $(X, \tau)$ é maximal, então $(X, \tau)$ é submaximal.

Demonstração. Seja $(X, \tau)$ um espaço topológico maximal e $T_{1}$. Por absurdo, suponhamos que o mesmo não seja submaximal. Existe, portanto, um subconjunto denso $D$ de $X$ que não é aberto em $X$. Consideremos $\tau^{\prime}$ a topologia sobre $X$ gerada pela subbase $\tau \cup\{D\}$. Claramente, $\tau^{\prime}$ é uma topologia estritamente mais fina do que $\tau$, uma vez que $D \in \tau^{\prime} \backslash \tau$.

Além disso, o espaço topológico $\left(X, \tau^{\prime}\right)$ é denso em si mesmo. Com efeito, como a família de todas as intersecções finitas de elementos de $\tau \cup\{D\}$ constitui uma base de 
abertos para o espaço topológico $\left(X, \tau^{\prime}\right)$, basta mostrar que uma tal intersecção ou é vazia, ou tem cardinalidade maior do que 1. Seja $U_{1} \cap \ldots \cap U_{n}$ uma intersecção finita de elementos de $\tau \cup\{D\}$. Se a mesma for vazia, nada temos a fazer. Suponhamos, portanto, que tal intersecção seja não vazia. Se $U_{i} \in \tau$, para todo $i \in\{1, \ldots, n\}$, então $U_{1} \cap \ldots \cap U_{n} \in \tau$ e, portanto, $\left|U_{1} \cap \ldots \cap U_{n}\right|>1$, já que $(X, \tau)$ é um espaço topológico maximal e, portanto, sem pontos isolados. Se existe $i \in\{1, \ldots, n\}$ tal que $U_{i}=D$, então $U_{1} \cap \ldots \cap U_{n}=U \cap D$, onde $U$ é um aberto não vazio de $(X, \tau)$. Como $(X, \tau)$ é um espaço topológico $T_{1}$, sem pontos isolados e $D$ é um subconjunto denso de $X$, segue que $|D \cap U| \geq \omega>1$. Logo, $\left(X, \tau^{\prime}\right)$ não tem pontos isolados, o que contradiz o fato de $(X, \tau)$ ser maximal. Portanto, $(X, \tau)$ é submaximal.

Teorema 3.1.9. Sejam $X$ um espaço topológico maximal, $U$ um subconjunto aberto de $X$ e $A$ um subconjunto qualquer de $X$ tais que $U \subset A \subset \bar{U}$. Então $A$ é, também, aberto em $X$.

Demonstração. Suponhamos, por absurdo, que $A$ não seja um subconjunto aberto de $(X, \tau)$. Consideremos $\tau^{\prime}$ a topologia sobre $X$ gerada pela subbase $\tau \cup\{A\}$. De nossa hipótese de absurdo, segue que $\tau^{\prime}$ é uma topologia estritamente mais fina do que $\tau$. Afirmamos, ainda, que $\left(X, \tau^{\prime}\right)$ é um espaço topológico denso em si mesmo.

Notemos, primeiramente, que o conjunto de todas as intersecções finitas de elementos de $\tau \cup\{A\}$ constitui uma base da abertos para o espaço topológico em questão. Portanto, basta mostrar que, se uma destas intersecções é não vazia, então a mesma contém mais de um ponto.

Seja $U_{1} \cap \ldots \cap U_{n}$ uma intersecção qualquer de elementos de $\tau \cup\{A\}$. Se, para todo $i \in\{1, \ldots, n\}$, tivermos que $U_{i} \in \tau$, então $U_{1} \cap \ldots \cap U_{n}$ é aberto de $(X, \tau)$ e, portanto, 
ou é vazio, ou tem cardinalidade maior do que 1, já que o espaço topológico em questão é maximal e, portanto, denso em si mesmo. Se, para algum $i \in\{1, \ldots, n\}$ tivermos que $U_{i}=A$, então $U_{1} \cap \ldots \cap U_{n}$ será um aberto de $A$ e podemos representá-lo como $V \cap A$, onde $V$ é um subconjunto aberto de $(X, \tau)$. Se $V \cap A=\emptyset$, nada temos a fazer. Caso contrário, teremos duas possibilidades: se $V \cap U=\emptyset$, então $V \cap \bar{U}=\emptyset$, um absurdo, já que $A \subset \bar{U}$. Logo, $V \cap U \neq \emptyset$ e, portanto, $|V \cap U|>1$, já que $V \cap U$ é aberto em $X$. Como $U \subset A$, segue que $|V \cap A|>1$. Portanto, $\left(X, \tau^{\prime}\right)$ não tem pontos isolados, o que contradiz a maximalidade de $(X, \tau)$. Logo, $A$ é aberto em $(X, \tau)$.

Corolário 3.1.10. O fecho de qualquer subconjunto aberto de um espaço topológico maximal é aberto e o interior de qualquer subconjunto fechado de um espaço topológico maximal é fechado.

Demonstração. Sejam $X$ um espaço topológico maximal e $U$ um subconjunto aberto de $X$. Temos que $U \subset \bar{U} \subset \bar{U}$ e, portanto, do teorema 3.1.9 segue que $\bar{U}$ é aberto em $X$. Consideremos, agora, $F$ um subconjunto fechado de $X$. Temos que $X \backslash F$ é aberto em $X$ e, portanto, $\overline{X \backslash F}$ também o é. Como int $(F)=X \backslash(\overline{X \backslash F})$, concluímos que int $(F)$ é fechado em $X$.

Mostramos, neste capítulo, que todo espaço topológico $T_{1}$ e maximal é, também, submaximal. Provamos, ainda, que todo espaço topológico submaximal é, em particular, HI. Evidentemente, todo espaço topológico HI é OHI e todo espaço topológico OHI é, por sua vez, irresolúvel. O restante desta seção será dedicado à construção de exemplos de espaços topológicos $T_{1}$ e sem pontos isolados que não verificam a recíproca das três últimas 
implicações. No capítulo 5, construiremos um exemplo de espaço topológico $T_{1}$, submaximal e denso em si mesmo, que não é maximal.

Proposição 3.1.11. Sejam $X$ um conjunto infinito, $\mathcal{F}$ um ultrafiltro livre sobre $X e$ $\tau=\mathcal{F} \cup\{\emptyset\}$. Então, $(X, \tau)$ é um espaço topológico denso em si mesmo, submaximal e $T_{1}$.

Demonstração. Mostremos, primeiramente, que $\tau$ é uma topologia sobre $X$. É evidente que $\emptyset \in \tau$ e que $X \in \tau$, já que $X \in \mathcal{F}$. Sejam $A, B \in \tau$. Se $A=\emptyset$ ou $B=\emptyset$, então $A \cap B=\emptyset$ e, portanto, $A \cap B \in \tau$. Se $A \neq \emptyset$ e $B \neq \emptyset$, então $A, B \in \mathcal{F}$. Como $\mathcal{F}$ é um filtro sobre $X$ segue que $A \cap B \in \mathcal{F} \subset \tau$. Por fim, seja $\left\{A_{i}\right\}_{i \in I}$ uma coleção de elementos de $\tau$. Se $A_{i}=\emptyset$, para todo $i \in I$, teremos que $\bigcup_{i \in I} A_{i}=\emptyset$ e, portanto, $\bigcup_{i \in I} A_{i} \in \tau$. Se existe $i_{0} \in I$ tal que $A_{i_{0}} \neq \emptyset$, então $A_{i_{0}} \in \mathcal{F}$ e, portanto, $\bigcup_{i \in I} A_{i} \in \mathcal{F} \subset \tau$, já que $A_{i_{0}} \subset \bigcup_{i \in I} A_{i}$. Logo, $\tau$ é uma topologia sobre $X$.

Seja $D$ um subconjunto denso de $X$. Então, $D \cap A \neq \emptyset$, para todo $A \in \tau \backslash\{\emptyset\}=\mathcal{F}$. Da proposição 1.1.5, segue que $D \in \mathcal{F} \subset \tau$. Logo, $X$ é submaximal.

Suponhamos, por absurdo, que $\{x\} \in \tau$, para algum $x \in X$. Então, $\{x\} \in \mathcal{F}$ e, como $\emptyset \notin \mathcal{F}$, temos que $\{x\} \cap A \neq \emptyset$, para todo $A \in \mathcal{F}$. Logo, $x \in A$, para todo $A \in \mathcal{F}$, o que é absurdo, em virtude da proposição 1.1.6. Portanto, $X$ é denso em si mesmo.

Provemos, finalmente, que $\{x\}$ é fechado em $X$, para todo $x \in X$. Seja $x$ um ponto qualquer de $X$. Já sabemos que $\{x\} \notin \mathcal{F}$. Do fato de $\mathcal{F}$ ser um ultrafiltro segue que $X \backslash\{x\} \in \mathcal{F} \subset \tau$. Logo, $\{x\}$ é fechado em $X$. Portanto, $X$ é $T_{1}$.

Notemos, ainda, que o espaço topológico acima apresentado não é $T_{2}$, uma vez que se $U$ e $V$ são abertos não vazios de $X$, então $U, V \in \mathcal{F}$ e, portanto, $U \cap V \in \mathcal{F}$, o que nos impede de ter $U \cap V=\emptyset$. 
Teorema 3.1.12. Existe um espaço topológico $T_{1}$, sem pontos isolados, que é irresolúvel, mas que não é OHI.

Demonstração. Sejam $Y$ e $Z$ conjuntos infinitos disjuntos. Seja $\mathcal{F}$ um ultrafiltro livre sobre $Y$. Munamos $Y$ da topologia $\sigma=\mathcal{F} \cup\{\emptyset\}$ e $Z$, da topologia cofinita $\rho$.

Consideremos $X=Y \cup Z$ e $\tau=\{A \cup B: A \in \sigma$ e $B \in \rho\}$.

Afirmamos que $\tau$ é uma topologia sobre $X$. De fato, como $\sigma$ e $\rho$ são topologias sobre $Y$ e $Z$, respectivamente, temos que $\emptyset, Y \in \sigma$ e $\emptyset, Z \in \rho$. Logo, $\emptyset, X \in \tau$. Sejam $A_{1} \cup B_{1}, A_{2} \cup B_{2} \in \tau$. Temos que

$$
\left(A_{1} \cup B_{1}\right) \cap\left(A_{2} \cup B_{2}\right)=\left(A_{1} \cap A_{2}\right) \cup\left(B_{1} \cap B_{2}\right)
$$

pois $Y \cap Z=\emptyset$. Como $\sigma$ e $\rho$ são topologias, segue que $A_{1} \cap A_{2} \in \sigma$ e $B_{1} \cap B_{2} \in \rho$. Logo, $\left(A_{1} \cup B_{1}\right) \cap\left(A_{2} \cup B_{2}\right) \in \tau$. Finalmente, seja $\left\{A_{i} \cup B_{i}\right\}_{i \in I}$ uma família de elementos de $\tau$. Temos que

$$
\bigcup_{i \in I}\left(A_{i} \cup B_{i}\right)=\bigcup_{i \in I} A_{i} \cup \bigcup_{i \in I} B_{i} .
$$

Como $\sigma$ e $\rho$ são topologias, segue que $\bigcup_{i \in I} A_{i} \in \sigma$ e $\bigcup_{i \in I} B_{i} \in \rho$. Logo, $\bigcup_{i \in I}\left(A_{i} \cup B_{i}\right) \in \tau$.

Portanto, $\tau$ é uma topologia sobre $X$.

Afirmamos que $(X, \tau)$ é um espaço topológico $T_{1}$. Com efeito, seja $x \in X$. Se $x \in Y$, então $\{x\} \in \mathcal{F}$ ou $Y \backslash\{x\} \in \mathcal{F}$, pois $\mathcal{F}$ é um ultrafiltro sobre $Y$. Como $\mathcal{F}$ é livre, $\{x\} \notin \mathcal{F}$ e, portanto, $Y \backslash\{x\} \in \mathcal{F}$. Logo, $Y \backslash\{x\} \in \sigma$ e, portanto

$$
X \backslash\{x\}=(Y \backslash\{x\}) \cup Z \in \tau
$$

Se $x \in Z$, então $\{x\}$ é fechado em $Z$, pois a topologia cofinita é $T_{1}$. Logo, $Z \backslash\{x\} \in \rho$ e, portanto,

$$
X \backslash\{x\}=Y \cup(Z \backslash\{x\}) \in \tau
$$


Afirmamos, ainda, que $X$ não possui pontos isolados. De fato, se houvesse $x \in X$ tal que $\{x\} \in \tau$, existiriam $A \in \sigma$ e $B \in \rho$ tais que $\{x\}=A \cup B$. Como $A \cap B=\emptyset$, ou $A=\{x\}$ e $B=\emptyset$ ou $A=\emptyset$ e $B=\{x\}$. O primeiro caso não pode ocorrer, pois $\mathcal{F}$ é livre. O segundo caso também não pode ocorrer, pois nenhum subconjunto finito é aberto em um conjunto infinito munido da topologia cofinita. Logo, $X$ não possui pontos isolados.

Mostremos que $X$ é um espaço topológico irresolúvel. De fato, se $X$ fosse resolúvel, todo subespaço aberto de $X$ também o seria. Claramente, $Y$ é um subespaço aberto de $X$. Contudo, em virtude do teorema 3.1.11, segue que $(Y, \sigma)$ é submaximal e $\sigma=\tau \uparrow Y$ (ou seja, $\sigma$ coincide com a topologia de subespaço induzida por $X$ em $Y$ ).

Contudo, $X$ não é OHI, pois $Z$ é um subespaço aberto resolúvel de $X$.

Teorema 3.1.13. Existe um espaço topológico $T_{1}$, sem pontos isolados, que é OHI, mas que não é $H I$.

Demonstração. Sejam $Y$ e $Z$ conjuntos infinitos disjuntos. Seja $\mathcal{F}$ um ultrafiltro livre sobre $Y$. Munamos $Y$ da topologia $\sigma=\mathcal{F} \cup\{\emptyset\}$ e $Z$ da topologia cofinita $\rho$.

Consideremos $X=Y \cup Z$ e $\tau=\{A \cup B: A \in \mathcal{F}$ e $B \in \rho\} \cup\{\emptyset\}$.

Afirmamos que $\tau$ é uma topologia sobre $X$. Claramente, $\emptyset \in \tau$. Como $Y \in \mathcal{F}$, pois $\mathcal{F}$ é um filtro sobre $Y$ e $Z \in \rho$, pois $\rho$ é uma topologia sobre $Z$, segue que $X=Y \cup Z \in \tau$. Sejam $A_{1} \cup B_{1}, A_{2} \cup B_{2} \in \tau \backslash\{\emptyset\}$. Temos que

$$
\left(A_{1} \cup B_{1}\right) \cap\left(A_{2} \cup B_{2}\right)=\left(A_{1} \cap A_{2}\right) \cup\left(B_{1} \cap B_{2}\right)
$$

já que $Y \cap Z=\emptyset$. Como $\mathcal{F}$ é um filtro e $\rho$ é uma topologia, segue que $A_{1} \cap A_{2} \in \mathcal{F}$ e $B_{1} \cap B_{2} \in \rho$. Logo, $\left(A_{1} \cup B_{1}\right) \cap\left(A_{2} \cup B_{2}\right) \in \tau$. Finalmente, seja $\left\{A_{i} \cup B_{i}\right\}_{i \in I}$ uma família de elementos de $\tau$. Se $A_{i} \cup B_{i}=\emptyset$, para todo $i \in I$, temos que $\bigcup_{i \in I}\left(A_{i} \cup B_{i}\right)=\emptyset \in \tau$. 
Suponhamos que exista $i_{0} \in I$ tal que $A_{i_{0}} \cup B_{i_{0}} \neq \emptyset$. Então, $A_{i_{0}} \in \mathcal{F}$. Temos que

$$
\bigcup_{i \in I}\left(A_{i} \cup B_{i}\right)=\bigcup_{i \in I} A_{i} \cup \bigcup_{i \in I} B_{i}
$$

Como $\mathcal{F}$ é um filtro e $\rho$ é uma topologia, segue que $\bigcup_{i \in I} A_{i} \in \mathcal{F}$ (pois $A_{i_{0}} \subset \bigcup_{i \in I} A_{i}$ ) e $\bigcup_{i \in I} B_{i} \in \rho$. Logo, $\bigcup_{i \in I}\left(A_{i} \cup B_{i}\right) \in \tau$.

Portanto, $\tau$ é uma topologia sobre $X$.

Afirmamos que $(X, \tau)$ é um espaço topológico $T_{1}$. Com efeito, seja $x \in X$. Se $x \in Y$, então $\{x\} \in \mathcal{F}$ ou $Y \backslash\{x\} \in \mathcal{F}$, pois $\mathcal{F}$ é um ultrafiltro sobre $Y$. Como $\mathcal{F}$ é livre, $\{x\} \notin \mathcal{F}$ e, portanto, $Y \backslash\{x\} \in \mathcal{F}$. Logo,

$$
X \backslash\{x\}=(Y \backslash\{x\}) \cup Z \in \tau
$$

Se $x \in Z$, então $\{x\}$ é fechado em $Z$, pois a topologia cofinita é $T_{1}$. Logo, $Z \backslash\{x\} \in \rho$ e, portanto

$$
X \backslash\{x\}=Y \cup(Z \backslash\{x\}) \in \tau
$$

Afirmamos, ainda, que $X$ não possui pontos isolados. De fato, se houvesse $x \in X$ tal que $\{x\} \in \tau$, existiriam $A \in \mathcal{F}$ (e, portanto, $A \neq \emptyset$ ) e $B \in \rho$ tais que $\{x\}=A \cup B$. Como $A \cap B=\emptyset$, segue que $A=\{x\}$ e $B=\emptyset$. Mas isso não pode acontecer, pois $\mathcal{F}$ é livre. Logo, $X$ não possui pontos isolados.

Mostremos que $X$ não é HI. De fato, $Z$ é um subespaço resolúvel de $X$, pois $\rho=\tau \uparrow Z$. Contudo, $X$ é OHI. Com efeito, seja $U \in \tau \backslash\{\emptyset\}$. Então, $U=A \cup B$, onde $A \in \mathcal{F}$ e $B \in \rho$. Como $Y \cap Z=\emptyset$, segue que $A=U \cap Y$. Como $Y$ é aberto em $X$, segue que $A$ é aberto em $U$. Se $U$ fosse resolúvel, $A$ também deveria ser. Contudo, $A$ não é resolúvel, pois $A$ é um subespaço aberto de $Y$ e $Y$ é submaximal e, portanto, OHI. Logo, $A$ é irresolúvel. Portanto, $U$ é irresolúvel e daí segue que $X$ é OHI. 
Teorema 3.1.14. Existe um espaço topológico $T_{1}$, sem pontos isolados, que é HI, mas que não é submaximal.

Demonstração. Sejam $Y$ e $Z$ conjuntos infinitos disjuntos. Sejam $\mathcal{F}$ um ultrafiltro livre sobre $Y$ e $\mathcal{G}$ um ultrafiltro livre sobre $Z$. Munamos $Y$ e $Z$ com as topologias $\sigma=\mathcal{F} \cup\{\emptyset\}$ e $\rho=\mathcal{G} \cup\{\emptyset\}$, respectivamente.

Consideremos $X=Y \cup Z$ e $\tau=\{A \cup B: A \in \mathcal{F}$ e $B \in \rho\} \cup\{\emptyset\}$.

Afirmamos que $\tau$ é uma topologia sobre $X$. De fato, é claro que $\emptyset \in \tau$. Além disso, $Y \in \mathcal{F}$, pois $\mathcal{F}$ é um filtro sobre $Y$ e $Z \in \rho$, pois $\rho$ é uma topologia sobre $Z$. Logo, $X=Y \cup Z \in \tau$. Sejam $A_{1} \cup B_{1}, A_{2} \cup B_{2} \in \tau \backslash\{\emptyset\}$. Temos que

$$
\left(A_{1} \cup B_{1}\right) \cap\left(A_{2} \cup B_{2}\right)=\left(A_{1} \cap A_{2}\right) \cup\left(B_{1} \cap B_{2}\right)
$$

uma vez que $Y \cap Z=\emptyset$. Como $\mathcal{F}$ é um filtro e $\rho$ é uma topologia, segue que $A_{1} \cap A_{2} \in \mathcal{F}$ e $B_{1} \cap B_{2} \in \rho$. Logo, $\left(A_{1} \cup B_{1}\right) \cap\left(A_{2} \cup B_{2}\right) \in \tau$. Finalmente, seja $\left\{A_{i} \cup B_{i}\right\}_{i \in I}$ uma família de elementos de $\tau$. Se $A_{i} \cup B_{i}=\emptyset$, para todo $i \in I$, temos que $\bigcup_{i \in I}\left(A_{i} \cup B_{i}\right)=\emptyset \in \tau$. Suponhamos que exista $i_{0} \in I$ tal que $A_{i_{0}} \cup B_{i_{0}} \neq \emptyset$. Então, $A_{i_{0}} \in \mathcal{F}$. Temos que

$$
\bigcup_{i \in I}\left(A_{i} \cup B_{i}\right)=\bigcup_{i \in I} A_{i} \cup \bigcup_{i \in I} B_{i}
$$

Como $\mathcal{F}$ é um filtro e $\rho$ é uma topologia, segue que $\bigcup_{i \in I} A_{i} \in \mathcal{F}$ (pois $A_{i_{0}} \subset \bigcup_{i \in I} A_{i}$ ) e $\bigcup_{i \in I} B_{i} \in \rho$. Logo, $\bigcup_{i \in I}\left(A_{i} \cup B_{i}\right) \in \tau$. Portanto, $\tau$ é uma topologia sobre $X$.

Afirmamos que $(X, \tau)$ é um espaço topológico $T_{1}$. Com efeito, seja $x \in X$. Se $x \in Y$, então $\{x\} \in \mathcal{F}$ ou $Y \backslash\{x\} \in \mathcal{F}$, pois $\mathcal{F}$ é um ultrafiltro sobre $Y$. Como $\mathcal{F}$ é livre, $\{x\} \notin \mathcal{F}$ e, portanto, $Y \backslash\{x\} \in \mathcal{F}$. Logo,

$$
X \backslash\{x\}=(Y \backslash\{x\}) \cup Z \in \tau
$$

Se $x \in Z$, então $\{x\} \in \mathcal{G}$ ou $Z \backslash\{x\} \in \mathcal{G}$, pois $\mathcal{G}$ é um ultrafiltro sobre $Z$. Como $\mathcal{G}$ é livre, 
$\{x\} \notin \mathcal{G}$ e, portanto, $Z \backslash\{x\} \in \mathcal{G}$. Logo,

$$
X \backslash\{x\}=Y \cup(Z \backslash\{x\}) \in \tau
$$

Afirmamos, ainda, que $X$ não possui pontos isolados. De fato, se houvesse $x \in X$ tal que $\{x\} \in \tau$, existiriam $A \in \mathcal{F}$ (e, portanto, $A \neq \emptyset)$ e $B \in \rho$ tais que $\{x\}=A \cup B$. Como $A \cap B=\emptyset$, então $A=\{x\}$ e $B=\emptyset$. Mas isso não pode acontecer, pois $\mathcal{F}$ é livre. Logo, $X$ não possui pontos isolados.

Mostremos que $X$ não é submaximal. De fato, $Y$ é um subconjunto denso de $X$ (pois se $U \in \tau \backslash\{\emptyset\}$, então $U=A \cup B$, onde $A \in \mathcal{F}$ e $B \in \rho$. Logo, $A \neq \emptyset$. Portanto, $\emptyset \neq A=A \cap Y=U \cap Y)$. Logo, para todo $x \in Z$, temos que $Y \subset Y \cup\{x\}$ e, portanto, $Y \cup\{x\}$ é denso em $X$. Mas $Y \cup\{x\}$ não é aberto em $X$ (isto é, não pertence a $\tau$ ), pois $\{x\}$ não pertence a $\rho$, já que $\rho$ não tem pontos isolados, de acordo com o teorema 3.1.11.

Todavia, $X$ é HI. De fato, seja $H \subset X$ um subespaço. Se $H \cap Y=\emptyset$, então $H \subset Z$ e, portanto, $H$ é irresolúvel, pois $Z$ é submaximal (devido ao teorema 3.1.11 e ao fato de $\tau \uparrow Z=\rho$ ) e, em particular, HI. Se $H \cap Y \neq \emptyset$, temos que $H \cap Y$ é aberto em $H$, pois $Y \in \tau$. Logo, se $H$ for resolúvel, $H \cap Y$ também o será. Contudo, $H \cap Y$ é, também, um subespaço de $Y$. Como $Y$ é submaximal (devido ao teorema 3.1.11 e ao fato de $\tau \uparrow Y=\sigma$ ), temos que $Y$ é, em particular, HI. Logo, $Y \cap H$ é irresolúvel. Portanto, $H$ é irresolúvel. Logo, $X$ é HI.

Observamos que os espaços topológicos exibidos nas demonstrações dos teoremas 3.1.12, 3.1 .13 e 3.1.14 não são $T_{2}$. Na seção seguinte, construiremos outros exemplos de espaços topológicos irresolúveis e sem pontos isolados, satisfazendo axiomas de separação mais convenientes. 


\subsection{Expansões de espaços topológicos}

Definição 3.2.1. Sejam $X_{1}=(X, \tau)$ e $X_{2}=\left(X, \tau^{\prime}\right)$ espaços topológicos de mesmo suporte. Dizemos que $X_{2}$ é uma expansão de $X_{1}$ se $\tau \subset \tau^{\prime}$, ou seja, se $\tau^{\prime}$ é uma topologia mais fina do que $\tau$.

Proposição 3.2.2. Sejam $X_{1}=(X, \tau)$ e $X_{2}=\left(X, \tau^{\prime}\right)$ espaços topológicos de mesmo suporte, tais que $X_{2}$ é uma expansão de $X_{1}$. Seja $P$ um subconjunto de $X$. O interior de $P$ com respeito a $X_{1}$ está contido no interior de $P$ com respeito a $X_{2}$ e o fecho de $P$ com respeito a $X_{2}$ está contido no fecho de $P$ com respeito a $X_{1}$. Além disso, se p é um ponto de acumulação de $P$ em $X_{2}$, então p é, também, um ponto de acumulação de $P$ em $X_{1}$.

Demonstração. Mostremos, primeiramente, que $\operatorname{int}_{X_{1}}(P) \subset \operatorname{int}_{X_{2}}(P)$. Com efeito, seja $p \in \operatorname{int}_{X_{1}}(P)$. Existe $U \in \tau$ tal que $p \in U \subset P$. Como $\tau \subset \tau^{\prime}$, temos que $U \in \tau^{\prime}$ e, portanto, $p \in \operatorname{int}_{X_{2}}(P)$.

Mostremos, agora, que $\mathrm{cl}_{X_{2}}(P) \subset \mathrm{cl}_{X_{1}}(P)$. De fato, seja $p \in \mathrm{cl}_{X_{2}}(P)$. Para todo $U \in \tau^{\prime}$ tal que $p \in U$, temos que $U \cap P \neq \emptyset$. Como $\tau \subset \tau^{\prime}$ segue, em particular, que para todo $U \in \tau$ tal que $p \in U$, vale que $U \cap P \neq \emptyset$. Portanto, $p \in \operatorname{cl}_{X_{1}}(P)$.

Provemos, finalmente, que se $p$ é um ponto de acumulação de $P$ em $X_{2}$, então $p$ é, também, um ponto de acumulação de $P$ em $X_{1}$. Com efeito, seja $p$ um ponto de acumulação de $P$ em $X_{2}$. Para todo $U \in \tau^{\prime}$ tal que $p \in U$, temos que $(U \backslash\{p\}) \cap P \neq \emptyset$. Como $\tau \subset \tau^{\prime}$ segue, em particular, que para todo $U \in \tau$ tal que $p \in U$, vale que $(U \backslash\{p\}) \cap P \neq \emptyset$. Logo, $p$ é ponto de acumulação de $P$ em $X_{1}$.

No próximo teorema, investigaremos o comportamento de determinadas propriedades referentes a espaços topológicos, quando submetidas ao processo de expansão. 
Teorema 3.2.3. Toda expansão de um espaço topológico $T_{0}, T_{1}$ ou $T_{2}$ é, ainda, um espaço topológico $T_{0}, T_{1}$ ou $T_{2}$, respectivamente. Entretanto, as propriedades de semi-regularidade, regularidade, regularidade completa, normalidade, metrizabilidade, compacidade e conexidade não são, em geral, preservadas por expansões de espaços topológicos.

Demonstração. Sejam $X_{1}=(X, \tau)$ e $X_{2}=\left(X, \tau^{\prime}\right)$ espaços topológicos de mesmo suporte, tais que $X_{2}$ é uma expansão de $X_{1}$.

Suponhamos que $X_{1}$ seja um espaço topológico $T_{0}$ e mostremos que $X_{2}$ também o é. Com efeito, sejam $x$ e $y$ elementos distintos de $X$. Por hipótese, existe $U \in \tau$ tal que $U$ contém um, e somente um, dos pontos $x$ e $y$. Como $\tau \subset \tau^{\prime}$, concluímos que $U \in \tau^{\prime}$ e, portanto, $X_{2}$ também é um espaço topológico $T_{0}$.

De maneira análoga, mostramos que se $X_{1}$ é um espaço topológico $T_{1}$ (respectivamente, $T_{2}$ ), então $X_{2}$ também é um espaço topológico $T_{1}$ (respectivamente, $T_{2}$ ).

Seja $I$ o intervalo fechado $[0,1]$ munido de sua topologia usual. Tal espaço é compacto, conexo e metrizável (e, em particular, é normal, completamente regular, regular e semiregular). Construiremos duas expansões do mesmo, uma que não é conexa e compacta e a outra que não é semi-regular (e, portanto, não é regular, completamente regular, normal ou metrizável)

Seja $S$ o conjunto $[0,1]$ munido da topologia discreta. Tal espaço topológico não é compacto - já que a coleção de todos os unitários de seus pontos constitui uma cobertura aberta do mesmo, que não admite subcobertura finita - e tampouco é conexo, pois cada um de seus "pontos" é aberto e fechado no espaço topológico em questão. Contudo, esta é, evidentemente, uma expansão de $I$.

Consideremos, agora, $T$ o espaço topológico que tem como suporte o conjunto $[0,1]$ e cujos abertos são quaisquer uniões de intervalos abertos em [0,1], dos quais fora retirada 
uma quantidade enumerável de pontos. Notemos que $T$ é uma expansão de $I$.

Afirmamos que $T$ não é semi-regular. De fato, se $U$ é um aberto não vazio de $T$, então $A \backslash B \subset U$, onde $A$ é um intervalo aberto em $[0,1]$ e $B$ é um subconjunto enumerável de $A . \quad \operatorname{Logo}, \operatorname{cl}_{T}(A \backslash B) \subset \mathrm{cl}_{T}(U)$. Afirmamos que $A \subset \operatorname{cl}_{T}(A \backslash B)$. Com efeito, seja $p \in A$. Como $B$ é enumerável, todo intervalo aberto em $[0,1]$ do qual fora retirada uma quantidade enumerável de pontos e que contém $p$ intersecta o conjunto $A \backslash B$. Portanto, $p \in \mathrm{cl}_{T}(A \backslash B)$. Logo, todo aberto regular contém um intervalo aberto em $[0,1]$ e, portanto, $T$ não possui uma base de abertos regulares (pois existem abertos em $T$ que não contém qualquer intervalo aberto em $[0,1])$. Em outras palavras, $T$ não é semi-regular.

O próximo teorema nos mostra que o axioma de separação de Urysohn é preservado por expansões de espaços topológicos.

Teorema 3.2.4. Toda expansão de um espaço topológico $T_{2 \frac{1}{2}}$ é, ainda, um espaço topológico $T_{2 \frac{1}{2}}$.

Demonstração. Seja $X_{1}=(X, \tau)$ um espaço topológico $T_{2 \frac{1}{2}}$. Consideremos $X_{2}=\left(X, \tau^{\prime}\right)$ uma expansão do espaço topológico $X_{1}$. Sejam $x$ e $y$ elementos distintos de $X$. Como $X_{1}$ é um espaço topológico $T_{2 \frac{1}{2}}$, existem $U, V \in \tau$ tais que $x \in U, y \in V \mathrm{e} \mathrm{cl}_{X_{1}}(U) \cap \mathrm{cl}_{X_{1}}(V)=\emptyset$. Como $\tau \subset \tau^{\prime}$, temos que $U, V \in \tau^{\prime}$. Da proposição 3.2.2 segue que $\operatorname{cl}_{X_{2}}(U) \subset \operatorname{cl}_{X_{1}}(U)$ e $\mathrm{cl}_{X_{2}}(V) \subset \mathrm{cl}_{X_{1}}(V)$. Logo, $\mathrm{cl}_{X_{2}}(U) \cap \mathrm{cl}_{X_{2}}(V)=\emptyset$. Portanto, $X_{2}$ é um espaço topológico $T_{2 \frac{1}{2}}$.

Não é difícil mostrar que todo espaço topológico regular é, em particular, um espaço de Urysohn. Contudo, o espaço topológico $T$ apresentado na demonstração do teorema 3.2.3 
é $T_{2 \frac{1}{2}}$ (já que é uma expansão de um espaço topológico $T_{2 \frac{1}{2}}$ ) que não é regular. Exemplos de espaços topológicos $T_{2}$ que não são $T_{2 \frac{1}{2}}$ podem ser encontrados em [15].

Teorema 3.2.5. Uma família qualquer de espaços topológicos de mesmo suporte constitui um sistema parcialmente ordenado com respeito à seguinte relação:

$$
X_{1} \prec X_{2} \text { se, e somente se } X_{2} \text { é uma expansão de } X_{1} \text {. }
$$

Toda família não vazia de espaços topológicos de mesmo suporte admite supremo com relação à ordem parcial .

Demonstração. Seja $\left\{\left(X, \tau_{i}\right)\right\}_{i \in I}$ uma família de espaços topológicos de mesmo suporte. Mostremos que $\prec$ é uma relação de ordem parcial no conjunto em questão. Com efeito, $\prec$ é reflexiva, pois um espaço topológico $\left(X, \tau_{i}\right)$ é sempre uma expansão de si próprio e, portanto, $\left(X, \tau_{i}\right) \prec\left(X, \tau_{i}\right)$.

Além disso, $\prec$ é anti-simétrica, pois se considerarmos $\left(X, \tau_{i}\right)$ e $\left(X, \tau_{j}\right)$ espaços topológicos de mesmo de mesmo suporte tais que $\left(X, \tau_{i}\right) \prec\left(X, \tau_{j}\right)$ e $\left(X, \tau_{j}\right) \prec\left(X, \tau_{i}\right)$, teremos que $\tau_{i} \subset \tau_{j}$ e $\tau_{j} \subset \tau_{i}$ e, portanto, $\tau_{i}=\tau_{j}$, ou seja, $\left(X, \tau_{i}\right)=\left(X, \tau_{j}\right)$. Finalmente, temos que $\prec$ é transitiva. De fato, dados espaços topológicos $\left(X, \tau_{i}\right),\left(X, \tau_{j}\right)$ e $\left(X, \tau_{k}\right)$ tais que $\left(X, \tau_{i}\right) \prec\left(X, \tau_{j}\right)$ e $\left(X, \tau_{j}\right) \prec\left(X, \tau_{k}\right)$, teremos que $\tau_{i} \subset \tau_{j}$ e $\tau_{j} \subset \tau_{k}$. Logo, $\tau_{i} \subset \tau_{k}$ e, portanto, $\left(X, \tau_{i}\right) \prec\left(X, \tau_{k}\right)$.

Isto encerra a demonstração de que $\prec$ é uma relação de ordem parcial. Resta mostrar que uma família não vazia de espaços topológicos de mesmo suporte admite supremo com relação à ordem parcial $\prec$.

Consideremos $\left\{\left(X, \tau_{i}\right)\right\}_{i \in I}$ uma família não vazia de espaços topológicos de mesmo suporte $X$. Seja $S=(X, \tau)$ o espaço topológico de suporte $X$, cuja topologia é gerada pela subbase $\bigcup_{i \in I} \tau_{i}$. Afirmamos que $S=(X, \tau)$ é o supremo de $\left\{\left(X, \tau_{i}\right)\right\}_{i \in I}$, com respeito à ordem parcial $\prec$. De fato, não há dúvidas de que $S=(X, \tau)$ é um limitante superior 
da família $\left\{\left(X, \tau_{i}\right)\right\}_{i \in I}$. Além disso, se $M=\left(X, \tau^{\prime}\right)$ for um limitante superior da família $\left\{\left(X, \tau_{i}\right)\right\}_{i \in I}$, é necessário que $\tau^{\prime}$ contenha $\tau$ e, portanto, $S \prec M$.

Relembramos que o caráter de dispersão de um espaço topológico $X$ é denotado por $\Delta(X)$ e é definido por

$$
\Delta(X)=\min \{|U|: U \text { é um aberto não vazio de } X\} .
$$

Teorema 3.2.6. Todo espaço topológico infinito e $T_{0}$ admite uma expansão que é um espaço topológico $T_{1}$ e que possui o mesmo caráter de dispersão do espaço topológico original.

Demonstração. Seja $X_{1}=(X, \tau)$ um espaço topológico infinito e $T_{0}$. Consideremos o espaço topológico $X_{2}$ de suporte $X$, munido da topologia cofinita. Sabemos que $X_{2}$ é um espaço topológico $T_{1}$. Consideremos $S=\left(X, \tau^{\prime}\right)$ o supremo da família $\left\{X_{1}, X_{2}\right\}$. Temos que $S$ é um espaço topológico $T_{1}$, já que é, em particular, uma expansão de $X_{2}$. Temos, também, que $S$ é uma expansão do espaço topológico $X_{1}$. Mostremos que $S$ possui o mesmo caráter de dispersão de $X_{1}$. Com efeito, se $\Delta\left(X_{1}\right)=1$, então $\Delta(S)=1$. Suponhamos, portanto, que $X_{1}$ tenha caráter de dispersão infinito (já que $X_{1}$ é $T_{0}$ ). Temos que $\tau^{\prime}$ é a topologia gerada pela subbase

$$
\tau \cup\{E \subset X: X \backslash E \text { é finito }\}
$$

Portanto, o conjunto das intersecções finitas de elementos de

$$
\tau \cup\{E \subset X: X \backslash E \text { é finito }\}
$$

constitui uma base de abertos para $S$. Qualquer uma destas intersecções finitas ou é um elemento de $\tau$, ou é um subconjunto de $X$ cujo complementar é finito, ou é da forma $U \cap E$, onde $U \in \tau$ e $E$ é um subconjunto de $X$ tal que $|X \backslash E|<\omega$. No primeiro caso, a 
cardinalidade de tal interseç̧ão é maior ou igual a $\Delta\left(X_{1}\right)$. No segundo caso, a cardinalidade de tal interseç̧ão é igual a $|X|$ e, portanto, é maior ou igual a $\Delta\left(X_{1}\right)$. No terceiro caso, a cardinalidade de $U \cap E$ é igual à cardinalidade de $U$, pois $U$ é infinito e retiramos de $U$, no máximo, um número finito de pontos. Logo, $|U \cap E| \geq \Delta\left(X_{1}\right)$. Isto prova que $\Delta(S)=\Delta\left(X_{1}\right)$.

Teorema 3.2.7. Todo espaço topológico infinito e $T_{2}$ admite uma expansão que é um espaço topológico $T_{2 \frac{1}{2}}$ e que possui o mesmo caráter de dispersão do espaço topológico original.

Demonstração. Seja $X_{1}=(X, \tau)$ um espaço topológico infinito e $T_{2}$ e consideremos $\left\{x_{i}\right\}_{i<|X|}$ uma indexação do conjunto $X$.

Se $x_{1}$ é separável de $x_{2}$ em $X_{1}$ (isto é, se existem abertos $U$ e $V$ de $X_{1}$ tais que $x_{1} \in U$,

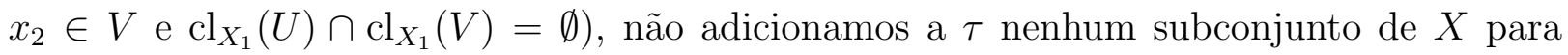
o par de pontos $\left(x_{1}, x_{2}\right)$. Suponhamos que $x_{1}$ não seja separável de $x_{2}$ em $X_{1}$. Como $X_{1}$ é $T_{2}$, existem abertos $U$ e $V$ de $X_{1}$ tais que $x_{1} \in U, x_{2} \in V$ e $U \cap V=\emptyset$. Portanto, $\operatorname{cl}_{X_{1}}(U) \cap V=\emptyset$ e, em particular, $x_{2} \notin \operatorname{cl}_{X_{1}}(U)$.

Se $W$ é um aberto de $X_{1}$ tal que $\bar{U} \cap W \neq \emptyset$, existe $x \in \bar{U} \cap W$. Como $x \in \bar{U}$ e $W$ é uma vizinhança aberta de $x$ em $X_{1}$, segue que $U \cap W \neq \emptyset$. Logo, $U \cap W$ é um aberto não vazio de $X_{1}$ e, portanto, $|U \cap W| \geq \Delta\left(X_{1}\right)$. Como $U \cap W \subset \bar{U} \cap W$, segue que $|\bar{U} \cap W| \geq \Delta\left(X_{1}\right)$.

Seja $X_{1,2}$ o espaço topológico que tem $X$ como suporte e cuja topologia é gerada pela subbase $\tau \cup\left\{\operatorname{cl}_{X_{1}}(U)\right\}$. Em virtude do parágrafo anterior segue que $\Delta\left(X_{1,2}\right)=\Delta\left(X_{1}\right)$. Afirmamos, ainda, que $x_{1}$ e $x_{2}$ são separáveis em $X_{1,2}$. De fato, $x_{1} \in \operatorname{cl}_{X_{1,2}}(U), x_{2} \in V$ e $\mathrm{cl}_{X_{1}}(U) \cap V=\emptyset$. Como $\mathrm{cl}_{X_{1}}(U)$ é aberto em $X_{1,2}$, segue que $\mathrm{cl}_{X_{1}}(U) \cap \mathrm{cl}_{X_{1,2}}(V)=\emptyset$. Logo, $\operatorname{cl}_{X_{1,2}}(U) \cap \operatorname{cl}_{X_{1,2}}(V)=\emptyset$. 
Ordenemos o conjunto dos pares de números ordinais da seguinte maneira:

$$
(\gamma, \delta) \lessdot(\alpha, \beta) \text { se, e somente se, vale que } \gamma<\alpha \text { ou vale que } \gamma=\alpha \text { e } \delta<\beta \text {. }
$$

Suponhamos terem sido definidos espaços topológicos $X_{\gamma, \delta}$ que verificam $\Delta\left(X_{\gamma, \delta}\right)=$ $\Delta\left(X_{1}\right)$ e tais que os pontos $x_{\gamma}$ e $x_{\delta}$ sejam separáveis em $X_{\gamma, \delta}$, para todos os pares de ordinais $(\gamma, \delta) \lessdot(\alpha, \beta)$.

Consideremos $S_{\alpha, \beta}$ o supremo da família $\left\{X_{\gamma, \delta}\right\}_{(\gamma, \delta) \lessdot(\alpha, \beta)}$. Temos que $\Delta\left(S_{\alpha, \beta}\right)=\Delta\left(X_{1}\right)$, já que $\Delta\left(X_{\delta, \eta}\right)=\Delta\left(X_{1}\right)$, para todo $(\delta, \eta) \lessdot(\alpha, \beta)$. Além disso, temos que quaisquer dois elementos $x_{\gamma}$ e $x_{\delta}$ de $X, \operatorname{com}(\gamma, \delta) \lessdot(\alpha, \beta)$ são separáveis em $S_{\alpha, \beta}$.

Se os pontos $x_{\alpha}$ e $x_{\beta}$ de $X$ forem separáveis em $S_{\alpha, \beta}$, façamos $X_{\alpha, \beta}=S_{\alpha, \beta}$. Se não, expandimos $S_{\alpha, \beta}$ da maneira acima descrita, de modo que $x_{\alpha}$ e $x_{\beta}$ sejam separáveis nesta expansão, a qual será denotada por $X_{\alpha, \beta}$.

Por indução transfinita, construímos os espaços $X_{\alpha, \beta}$.

Seja $X_{2}$ o supremo de todos os espaços $X_{\alpha, \beta}$ em questão. Temos que $X_{2}$ é uma expansão de $X_{1}$. Além disso, $\Delta\left(X_{2}\right)=\Delta\left(X_{1}\right)$ e $X_{2}$ é $T_{2 \frac{1}{2}}$.

Estudaremos, no próximo teorema, a preservação de alguns axiomas de separação pelo supremo de uma família de espaços topológicos.

Teorema 3.2.8. Seja $\left\{X_{i}\right\}_{i \in I}$ uma família não vazia de espaços topológicos de mesmo suporte, totalmente ordenada pela relação $\prec$. Se, para algum $i \in I$, o espaço topológico $X_{i}$ é $T_{0}, T_{1}, T_{2}$ ou $T_{2 \frac{1}{2}}$, então o supremo $S$ da família em questão é, também, um espaço topológico $T_{0}, T_{1}, T_{2}$ ou $T_{2 \frac{1}{2}}$, respectivamente. Se, para todo $i \in I$, o espaço topológico $X_{i}$ for regular (respectivamente, completamente regular), então $S$ também será regular (respectivamente, completamente regular). 
Demonstração. A primeira afirmação decorre dos teoremas 3.2.3 e 3.2.4.

Suponhamos, agora, que para todo $i \in I$, o espaço topológico $X_{i}$ seja regular. Mostremos que $S$ também o será. Seja $G$ um aberto qualquer de $S$. Fazendo $X_{i}=\left(X, \tau_{i}\right)$, para todo $i \in I$, temos que

$$
G=\bigcup_{\lambda \in \Lambda} G_{\alpha_{1(\lambda)}} \cap \ldots \cap G_{\alpha_{n(\lambda)}}
$$

onde cada $G_{\alpha_{i(\lambda)}} \in \tau_{i(\lambda)}$ e $i(\lambda) \in I$.

Como $\left\{X_{i}\right\}_{i \in I}$ é totalmente ordenado pela relação $\prec$, temos que

$$
G=\bigcup_{\alpha \in A} G_{\alpha}
$$

onde $A \subset I$ e cada $G_{\alpha}$ é aberto em $X_{\alpha}$.

Se $p$ é um ponto qualquer de $G$, existe $\alpha_{0} \in A$ tal que $p \in G_{\alpha_{0}}$. Como $X_{\alpha_{0}}$ é regular, por hipótese, existe um aberto $H$ de $X_{\alpha_{0}}$ tal que

$$
p \in H \subset \operatorname{cl}_{X_{\alpha_{0}}}(H) \subset G_{\alpha_{0}} \subset G \text {. }
$$

Da proposição 3.2.2 segue que $\operatorname{cl}_{S}(H) \subset \operatorname{cl}_{X_{\alpha_{0}}}(H)$. Logo,

$$
p \in H \subset \operatorname{cl}_{S}(H) \subset G_{\alpha_{0}} \subset G
$$

Portanto, $S$ é $T_{3}$. Como $S$ é $T_{1}$, segue que $S$ é regular.

Suponhamos, agora, que para todo $i \in I$, o espaço topológico $X_{i}$ seja completamente regular. Mostremos que $S$ também o será. Seja $F$ um subconjunto fechado de $S$ e $p$ um ponto de $S$ que não pertence a $F$. Então, $G=X \backslash F$ é um aberto de $S$ ao qual $p$ pertence.

Repetindo o argumento utilizado acima, concluímos que existe $\alpha_{0} \in I$ tal que $p \in G_{\alpha_{0}}$, para algum $G_{\alpha_{0}} \subset G$ aberto em $X_{\alpha_{0}}$.

Como $X_{\alpha_{0}}$ é regular, existe

$$
f: X_{\alpha_{0}} \rightarrow[0,1]
$$


contínua tal que $f(p)=0$ e $f(q)=1$, para todo $q \in X \backslash G_{\alpha_{0}}$.

Como $S$ é uma expansão de $X_{\alpha_{0}}$, temos que a função $f$ exibida acima, considerada agora com domínio $S$, também é contínua.

Além disso, $f(F) \subset\{1\}$, pois $F \subset X \backslash G_{\alpha_{0}}$. Logo, $S$ é $T_{3 \frac{1}{2}}$. Como $S$ é $T_{1}$, segue que $S$ é completamente regular.

Definição 3.2.9. Seja $\kappa>1$ um cardinal. Um espaço topológico $X$ que tem caráter de dispersão maior ou igual a $\kappa$ e tal que toda expansão própria de $X$ tem caráter de dispersão menor que $\kappa$ é dito $\kappa$-maximal.

Observamos que um espaço topológico $T_{0}$ é $\omega$-maximal se, e somente se, é maximal.

Definição 3.2.10. Seja $\mathcal{P}$ uma propriedade topológica. Se toda expansão de um espaço topológico que goza da propriedade $\mathcal{P}$ também a verifica, dizemos que $\mathcal{P}$ é invariante por expansões.

Teorema 3.2.11. Sejam $\kappa$ um cardinal infinito e $\mathcal{P}$ uma propriedade válida para espaços topológicos, invariante por expansões. Todo espaço topológico $T_{0}$ que goza da propriedade $\mathcal{P}$ e cujo caráter de dispersão é maior ou igual a $\kappa$ admite uma expansão $\kappa$-maximal que também goza da propriedade $\mathcal{P}$.

Demonstração. Seja $E$ o suporte de um espaço topológico $X=(E, \tau)$ que é $T_{0}$, goza da propriedade $\mathcal{P}$ e cujo caráter de dispersão é maior ou igual a $\kappa$.

Seja $\mathcal{B}$ a coleção das subfamílias de $\mathcal{P}(E)$ que são bases de abertos para espaços topológicos que são expansões de $X$, que gozam de $\mathcal{P}$ e que tem caráter de dispersão maior ou igual a $\kappa$, ordenada pela inclusão. 
Seja $\mathcal{C}=\left\{B_{i}\right\}_{i \in I}$ uma cadeia em $\mathcal{B}$. Seja $Y$ o espaço topológico de suporte $E$ que tem como base de abertos $\bigcup_{i \in I} B_{i}$. Notemos que $\bigcup_{i \in I} B_{i}$ é, de fato, base de abertos para alguma topologia sobre $E$ (mais fina do que $\tau$ ), pois $\mathcal{C}$ é uma cadeia. Temos que $\Delta(Y) \geq \kappa$. De fato, se $U$ é um aberto não vazio de $Y$, então $U \supset U_{i}$, para algum $U_{i} \in B_{i}$ e, portanto, $|U| \geq\left|U_{i}\right| \geq \kappa$. Logo, $\mathcal{C}$ é majorada por $Y$.

Do lema de Kuratowski-Zorn segue que $\mathcal{B}$ possui um elemento maximal $\mathcal{M}$ de $\mathcal{B}$. Seja $(E, \sigma)$ o espaço topológico que tem $\mathcal{M}$ como base de abertos. Temos que $(E, \sigma)$ é uma expansão de $X$ que tem caráter de dispersão maior ou igual a $\kappa$ e, claro, goza de $\mathcal{P}$, pois $\mathcal{P}$ é invariante por expansões. Além disso, qualquer expansão própria $(E, \rho)$ de $(E, \sigma)$ terá caráter de dispersão menor do que $\kappa$, em virtude da maximalidade de $\mathcal{M}$.

Teorema 3.2.12. Se $\kappa$ é um cardinal infinito, então todo espaço topológico $T_{0}$ cujo caráter de dispersão é maior ou igual a $\kappa$ admite uma expansão $\kappa$-maximal que é, necessariamente, um espaço topológico $T_{1}$. Além disso, todo espaço topológico $T_{2}$ cujo caráter de dispersão é maior ou igual a $\kappa$ admite uma expansão $\kappa$-maximal que é, necessariamente, um espaço topológico $T_{2 \frac{1}{2}}$.

Demonstração. Segue dos teoremas 3.2.6, 3.2.7 e 3.2.11.

Teorema 3.2.13. Seja $\kappa$ um cardinal infinito. Existe um espaço topológico $\kappa$-maximal que é $T_{2 \frac{1}{2}}$.

Demonstração. Em vista do teorema 3.2.12, basta mostrar que existe um espaço topológico $T_{2}$ de caráter de dispersão maior ou igual a $\kappa$. Seja

$$
Y=\{f: \omega \rightarrow \kappa \mid\{n \in \omega: f(n) \neq 0\} \text { é finito }\} \subset \kappa^{\omega} .
$$


Como $\kappa^{\omega}$ é metrizável, segue que $Y$ também o é e, portanto, $Y$ é $T_{2}$.

Além disso, temos que $|Y|=\kappa$ e $\Delta(Y)=\kappa$.

Definição 3.2.14. Seja $\kappa>1$ um cardinal infinito e seja $\mathcal{P}$ uma propriedade válida para espaços topológicos. Um espaço topológico $X$ que goza da propriedade $\mathcal{P}$, que verifica $\Delta(X) \geq \kappa$ e tal que qualquer expansão própria de $X$ ou não mais satisfaz a propriedade $\mathcal{P}$ ou tem caráter de dispersão menor do que $\kappa$ é dito $\kappa$-maximal com respeito à propriedade $\mathcal{P}$.

Definição 3.2.15. Seja $\mathcal{P}$ uma propriedade topológica. Se o supremo de toda família de espaços topológicos que gozam de $\mathcal{P}$, totalmente ordenada pela relação $\prec$, também verifica $\mathcal{P}$, dizemos que $\mathcal{P}$ é uma propriedade de expansão monotônica.

Teorema 3.2.16. Seja $\kappa$ um cardinal infinito. Se $\mathcal{P}$ é uma propriedade de expansão monotônica, então todo espaço topológico que goza de $\mathcal{P}$ e cujo caráter de dispersão é maior ou igual a $\kappa$ admite uma expansão $\kappa$-maximal com respeito à propriedade $\mathcal{P}$.

Demonstração. Seja $E$ o suporte de um espaço topológico $X=(E, \tau)$ que goza da propriedade $\mathcal{P}$ e cujo caráter de dispersão é maior ou igual a $\kappa$.

Seja $\mathcal{B}$ a coleção das subfamílias de $\mathcal{P}(E)$ que são bases de abertos para espaços topológicos que são expansões de $X$, que gozam de $\mathcal{P}$ e que tem caráter de dispersão maior ou igual a $\kappa$, ordenada pela inclusão.

Seja $\mathcal{C}=\left\{B_{i}\right\}_{i \in I}$ uma cadeia em $\mathcal{B}$. Consideremos $\tau_{i}$ a topologia sobre $E$ que tem $B_{i}$ como base de abertos. Seja $S=(E, \sigma)$ o supremo da família $\left\{\left(E, \tau_{i}\right)\right\}_{i \in I}$. Como $\mathcal{P}$ é uma propriedade de expansão monotônica, segue que $S$ goza de $\mathcal{P}$. Além disso, $\Delta(S) \geq \kappa$. Portanto, $S$ majora $\mathcal{C}$. 
Do lema de Kuratowski-Zorn segue que existe um elemento maximal $\mathcal{M}$ de $\mathcal{B}$. Seja $(E, \sigma)$ o espaço topológico que tem $\mathcal{M}$ como base de abertos. Temos que $(E, \sigma)$ é uma expansão de $X$ que goza de $\mathcal{P}$ e cujo caráter de dispersão é maior ou igual a $\kappa$. Além disso, qualquer expansão própria $(E, \rho)$ de $(E, \sigma)$ terá caráter de dispersão menor do que $\kappa$, ou não verificará a propriedade $\mathcal{P}$, em virtude da maximalidade de $\mathcal{M}$.

Teorema 3.2.17. Se $\kappa$ é um cardinal infinito, então todo espaço topológico regular cujo caráter de dispersão é maior ou igual a $\kappa$ admite uma expansão que é $\kappa$-maximal com respeito à propriedade de ser regular. Além disso, todo espaço topológico completamente regular cujo caráter de dispersão é maior ou igual a $\kappa$ admite uma expansão que é $\kappa$-maximal com respeito à propriedade de ser completamente regular.

Demonstração. Segue dos teoremas 3.2.8 e 3.2.16.

Teorema 3.2.18. Seja $\kappa$ um cardinal infinito. Existem espaços topológicos $\kappa$-maximais com respeito às propriedades de regularidade e de regularidade completa.

Demonstração. Em vista do teorema 3.2.17, basta mostrar que existem espaços topológicos regulares e completamente regulares de caráter de dispersão maior ou igual a $\kappa$. O espaço topológico considerado no teorema 3.2.13 possui estas características.

Teorema 3.2.19. Todo espaço topológico $T_{0}$ e denso em si mesmo admite uma expansão que é um espaço topológico $T_{1}$ e submaximal. Todo espaço topológico $T_{2}$ e denso em si mesmo admite uma expansão que é um espaço topológico $T_{2 \frac{1}{2}}$ e submaximal. 
Demonstração. Basta aplicar o teorema 3.2.12 para $\kappa=\omega$ e utilizar o teorema 3.1.8.

Teorema 3.2.20. Seja $\kappa$ um cardinal infinito. Existe um espaço topológico de cardinalidade $\kappa$ que é $T_{2 \frac{1}{2}}$ e submaximal.

Demonstração. Basta aplicar o teorema 3.2.19 ao espaço considerado no teorema 3.2.13.

Teorema 3.2.21. Todo espaço topológico $\omega$-maximal com respeito à propriedade de ser completamente regular é hereditariamente irresolúvel.

Demonstração. Seja $(X, \tau)$ um espaço topológico $\omega$-maximal com respeito à propriedade de ser completamente regular. Suponhamos, por absurdo, que $X$ não seja HI. Então, existe $A \subset X$ resolúvel na topologia induzida. Da proposição 2.1 .5 segue que $\bar{A}$ é, também, resolúvel na topologia induzida e, portanto, contém dois subconjuntos densos complementares $P$ e $(X \backslash P) \cap \bar{A}$. Seja $\left\{U_{i}\right\}_{i \in I}$ uma indexação de $\tau$.

Consideremos $\sigma$ a topologia gerada pela subbase

$$
\tau \cup\{P\} \cup\{(X \backslash P) \cap \bar{A}\}
$$

Temos que $(X, \sigma)$ é uma expansão de $(X, \tau)$ e

$$
\tau \cup\left\{P \cap U_{i}\right\}_{i \in I} \cup\left\{[(X \backslash P) \cap \bar{A}] \cap U_{i}\right\}_{i \in I}
$$

é uma base de abertos para $(X, \sigma)$.

Afirmamos que $(X, \sigma)$ é um espaço topológico denso em si mesmo. Com efeito, basta mostrar que se $U \in \tau$ e $U \cap P \neq \emptyset$ (respectivamente, $U \cap[(X \backslash P) \cap \bar{A}] \neq \emptyset$ ), então $|U \cap P|>1$ 
(respectivamente, $|U \cap[(X \backslash P) \cap \bar{A}]|>1$ ). Se $U \cap P \neq \emptyset$, então $U \cap \bar{A} \neq \emptyset$, pois $P \subset \bar{A}$. Logo, $U \cap \bar{A}$ é um subconjunto aberto não vazio de $\bar{A}$. Como $P$ é denso em $\bar{A}$ e $\bar{A}$ é, em particular, $T_{1}$ (já que $X$ é completamente regular), temos que $|P \cap(U \cap \bar{A})|=|P \cap U| \geq \omega>1$. Analogamente, mostramos que $|U \cap[(X \backslash P) \cap \bar{A}]|>1$.

Seja $\psi_{B}: X \rightarrow[0,1]$ tal que $\psi_{B}(B) \subset\{0\}$ e $\psi_{B}(X \backslash B) \subset\{1\}$, onde $B$ é um subconjunto arbitrário e fixado de $X$.

Como $P$ e $X \backslash P=[(X \backslash P) \cap \bar{A}] \cup(X \backslash \bar{A})$ são abertos em $(X, \sigma)$, segue que a função

$$
\psi_{P}:(X, \sigma) \rightarrow[0,1]
$$

é contínua. Analogamente, concluímos que a função

$$
\psi_{[(X \backslash P) \cap \bar{A}]}:(X, \sigma) \rightarrow[0,1]
$$

é contínua.

Se $U_{i}$ é um subconjunto aberto não vazio qualquer de $(X, \tau)$ e se $p$ é um ponto arbitrário de $U_{i}$, existe, em virtude da regularidade completa do espaço topológico em questão, uma função contínua

$$
f_{p, U_{i}}:(X, \tau) \rightarrow[0,1]
$$

tal que $f_{p, U_{i}}(p)=0$ e $f_{p, U_{i}}(q)=1$, para todo $q \in X \backslash U_{i}$.

Como $(X, \sigma)$ é uma expansão de $(X, \tau)$, segue que

$$
f_{p, U_{i}}:(X, \sigma) \rightarrow[0,1]
$$

é, também, uma função contínua.

Seja $H$ um aberto básico qualquer de $(X, \sigma)$ e $p$ um ponto qualquer de $H$. Se $H=U_{i}$, definimos

$$
g_{p, H}=f_{p, U_{i}}
$$


Se $H=P \cap U_{i}$, definimos

$$
g_{p, H}=\max \left\{\psi_{P}, f_{p, U_{i}}\right\}
$$

Se $H=[(X \backslash P) \cap \bar{A}] \cap U_{i}$, definimos

$$
g_{p, H}=\max \left\{\psi_{[(X \backslash P) \cap \bar{A}]}, f_{p, U_{i}}\right\}
$$

As funções $g_{p, H}$ acima definidas mostram que $(X, \sigma)$ é completamente regular.

Como $(X, \sigma)$ é denso em si mesmo e completamente regular, segue que seu caráter de dispersão é infinito. Do fato de $(X, \tau)$ ser $\omega$-maximal com respeito à propriedade de ser completamente regular vem que $(X, \sigma)=(X, \tau)$. Portanto, $P$ e $(X \backslash P) \cap \bar{A}$ são abertos disjuntos em $(X, \tau)$, o que contradiz o fato de ambos serem densos em $\bar{A}$. Logo, $(X, \tau)$ é HI.

Teorema 3.2.22. Todo espaço topológico completamente regular e denso em si mesmo admite uma expansão completamente regular, densa em si mesma e hereditariamente irresolúvel.

Demonstração. Basta aplicar o teorema 3.2.17 para $\kappa=\omega$ e, em seguida, utilizar o teorema 3.2.21.

Teorema 3.2.23. Seja $\kappa$ um cardinal infinito. Existe um espaço topológico completamente regular, denso em si mesmo e hereditariamente irresolúvel de cardinalidade $\kappa$.

Demonstração. Basta aplicar o teorema 3.2.22 ao espaço topológico considerado no teorema 3.2.13. 


\section{Capítulo 4}

\section{Resolubilidade finita e}

\section{$\omega$-resolubilidade}

Neste capítulo, provaremos que dado $n>1$ um número natural, existe um espaço topológico que é $n$-resolúvel, mas que não é $(n+1)$-resolúvel. A demonstração apresentada nesta dissertação foi extraída de [5]. Uma prova alternativa do mesmo resultado encontrase em [6]. Mostraremos, contudo, que se um espaço topológico for $n$-resolúvel, para todo número natural $n>1$, o mesmo será $\omega$-resolúvel. As demonstrações dos lemas 4.3 e 4.4 foram publicadas em [6] e as demonstrações do lema 4.5 e do teorema 4.6 foram publicadas em [9]. 
Lema 4.1. Seja X um espaço topológico irresolúvel. Existe um subespaço aberto não vazio e $H I$ de $X$.

Demonstração. Decorre imediatamente do teorema 2.1.9.

Teorema 4.2. Seja $n>1$ um número natural. Existe um espaço topológico enumerável, regular, que é n-resolúvel, mas que não é $(n+1)$-resolúvel.

Demonstração. Seja $n>1$ um número natural. Observamos, a princípio, que se existe $X$ um espaço topológico enumerável e regular, que é $n$-resolúvel, mas que não é $(n+1)$ resolúvel, então, para todo $1<k \leq n$ existe $Y$ um espaço topológico enumerável e regular, que é $k$-resolúvel, mas que não é $(k+1)$-resolúvel. Com efeito, fixemos $D_{1}, \ldots, D_{n}$ subconjuntos densos e dois a dois disjuntos de $X$ e façamos $Y=D_{1} \cup \ldots \cup D_{k}$. É claro que $Y$ é enumerável, regular e $k$-resolúvel. Se $Y$ fosse $(k+1)$-resolúvel, existiriam $E_{1}, \ldots, E_{k+1}$ subconjuntos densos e dois a dois disjuntos de $Y$. Afirmamos que $E_{i}$ é denso em $X$, para todo $i \in\{1, \ldots, k+1\}$. De fato, seja $U$ um aberto não vazio de $X$. Como $D_{1}$ é denso em $X$, temos que $U \cap D_{1} \neq \emptyset$ e, portanto, $U \cap Y \neq \emptyset$. Como $E_{i}$ é denso em $Y$ temos que $E_{i} \cap(U \cap Y) \neq \emptyset$ e, em particular, $E_{i} \cap U \neq \emptyset$. Como $E_{i} \subset Y$, temos que $E_{i} \cap D_{j}=\emptyset$, se $j>k$. Logo, $X$ terá $(n+1)$ subconjuntos densos disjuntos (a saber, $E_{1}, \ldots, E_{k+1}, D_{k+1}, \ldots, D_{n}$ ), o que é absurdo, já que $X$ não é $(n+1)$-resolúvel. Portanto, é suficiente mostrar que, para cada número natural $n \geq 1$, existe um espaço topológico enumerável e regular que é $2^{n}$-resolúvel, mas que não é $\left(2^{n}+1\right)$-resolúvel.

Seja $\mathcal{I}$ uma família infinita, independente e maximal de subconjuntos de $\omega$ tal que, para cada $p, q \in \omega, \operatorname{com} p \neq q$, o conjunto

$$
\{I \in \mathcal{I}:|\{p, q\} \cap I|=1\}
$$


seja infinito. Seja $\tau$ a topologia em $\omega$ gerada pela subbase

$$
\mathcal{I} \cup\{\omega \backslash I: I \in \mathcal{I}\}
$$

Afirmamos que $X=(\omega, \tau)$ é um espaço topológico enumerável, regular e irresolúvel. De fato, sejam $p$ e $q$ pontos distintos de $X$. Sabemos que existe $I \in \mathcal{I}$ tal que $|\{p, q\} \cap I|=1$. Como $I$ e $\omega \backslash I$ são abertos em $X$, concluímos que $X$ é um espaço topológico $T_{2}$. Uma vez que cada elemento da subbase acima descrita é aberto e fechado no espaço topológico em questão, segue que o mesmo é regular. Mostremos, por fim, que $X$ é irresolúvel. Para tanto, seja $D$ um subconjunto denso de $X$. Como $X$ é um espaço topológico $T_{1}$, a intersecção de $D$ com qualquer aberto básico de $X$ é um conjunto infinito. Portanto, se $\omega \backslash D$ também fosse denso em $X$, teríamos que $D \in \mathcal{I}$, devido à maximalidade de $\mathcal{I}$. Assim, $D$ seria um subconjunto aberto de $X$, o que contradiz o fato de seu complementar ser denso de $X$. Logo, $X$ não possui dois subconjuntos densos complementares, ou seja, $X$ é irresolúvel.

Como $X$ é um espaço topológico zero-dimensional, do lema 4.1 decorre que existe um subespaço aberto e fechado $U$ de $X$, que é HI. Existem, ainda, $\mathcal{A}$ e $\mathcal{B}$ subconjuntos finitos e disjuntos de $\mathcal{I}$ tais que

$$
\bigcap \mathcal{A} \backslash \bigcup \mathcal{B} \subset U
$$

Seja

$$
\mathcal{I}^{\prime}=\{I \cap U: I \in \mathcal{I} \backslash(\mathcal{A} \cup \mathcal{B})\}
$$

Não é difícil mostrar que $\mathcal{I}^{\prime}$ é uma família infinita e independente de subconjuntos de $U$. Consideremos $\tau^{\prime}$ a topologia gerada pela subbase

$$
\mathcal{I}^{\prime} \cup\left\{U \backslash I: I \in \mathcal{I}^{\prime}\right\}
$$

Temos que $\left(U, \tau^{\prime}\right)$ é um espaço topológico enumerável, hereditariamente irresolúvel e regular, pois $\mathcal{A}$ e $\mathcal{B}$ são finitos. 
Fixemos $n$ um número natural positivo e consideremos $\mathcal{F} \subset \mathcal{I}^{\prime}$ tal que $|\mathcal{F}|=n$. Seja $\sigma$ a topologia sobre o conjunto $U$ que tem

$$
\left(\mathcal{I}^{\prime} \backslash \mathcal{F}\right) \cup\left\{U \backslash I: I \in \mathcal{I}^{\prime} \backslash \mathcal{F}\right\}
$$

como subbase. Evidentemente,

$$
\mathcal{D}=\{\bigcap \mathcal{G} \backslash \bigcup(\mathcal{F} \backslash \mathcal{G}): \mathcal{G} \subset \mathcal{F}\}
$$

é uma coleção de subconjuntos densos e dois a dois disjuntos de $(U, \sigma)$. Logo, $(U, \sigma)$ é um espaço topológico enumerável, regular e $2^{n}$-resolúvel.

Seja $\mathcal{K}$ uma coleção qualquer de subconjuntos dois a dois disjuntos de $U$, tal que $|\mathcal{K}| \geq 2^{n}+1$. Mostraremos que nem todos os elementos de $\mathcal{K}$ são densos em $(U, \sigma)$.

Seja $D \in \mathcal{D}$. Então, $D=\bigcap \mathcal{G} \backslash \bigcup(\mathcal{F} \backslash \mathcal{G})$, para algum $\mathcal{G} \in \mathcal{F}$. Como $\left(U, \tau^{\prime}\right)$ é HI, concluímos que nem todos os elementos de $\mathcal{K}$ são densos em $D$. Portanto, existe um subconjunto aberto não vazio de $D$ que não intersecta pelo menos um elemento de $\mathcal{K}$. Prosseguindo desta maneira, encontramos $D^{\prime}$ um subconjunto aberto não vazio de $D$ que intersecta, no máximo, um elemento de $\mathcal{K}$. Podemos supor que $D^{\prime}$ é da forma $D \cap[(\bigcap \mathcal{A}) \backslash(\bigcup \mathcal{B})]$, onde $\mathcal{A}$ e $\mathcal{B}$ são subconjuntos finitos e disjuntos de $\mathcal{I}^{\prime} \backslash \mathcal{F}$.

Como $\mathcal{D}$ é finito, é possível encontrar subconjuntos finitos e disjuntos $\mathcal{L}, \mathcal{M} \subset \mathcal{I}^{\prime} \backslash \mathcal{F}$ tais que, para todo $D \in \mathcal{D}, D \cap[(\cap \mathcal{L}) \backslash(\bigcup \mathcal{M})]$ intersecta, no máximo, um elemento de $\mathcal{K}$. Uma vez que $\mathcal{D}$ recobre $U$, segue que $(\bigcap \mathcal{L}) \backslash(\bigcup \mathcal{M})$ intersecta, no máximo, $|\mathcal{D}|$ elementos de $\mathcal{K}$. Mas $|\mathcal{D}|<|\mathcal{K}|$ e, portanto, existe $K \in \mathcal{K}$ tal que

$$
[(\bigcap \mathcal{L}) \backslash(\bigcup \mathcal{M})] \cap K=\emptyset
$$

Como $(\bigcap \mathcal{L}) \backslash(\bigcup \mathcal{M})$ é um subconjunto aberto não vazio de $(U, \sigma)$, segue que $K$ não é denso em $(U, \sigma)$. Portanto, $(U, \sigma)$ não é um espaço topológico $\left(2^{n}+1\right)$-resolúvel. 
Apresentaremos, a seguir, três lemas que serão utilizados para mostrar que se um espaço topológico é $n$-resolúvel, para todo número natural $n>1$, então o mesmo também é $\omega$ resolúvel.

Lema 4.3. Sejam $X$ um espaço topológico e $n>1$ um número natural. Se $X$ é $(n+1)$ resolúvel e $Y$ é um subespaço $O H I$ de $X$, então $X \backslash Y$ é n-resolúvel e denso em $X$.

Demonstração. Provaremos, primeiramente, que $X \backslash Y$ é um subconjunto denso de $X$. Caso contrário, existiria um aberto não vazio $U$ de $X$ tal que $U \cap(X \backslash Y)=\emptyset$ ou seja, tal que $U \subset Y$. Como $X$ é resolúvel, da proposição 2.1.7 segue que $U$ também o é. Como $U$ é, também, aberto em $Y$, segue que $U$ é irresolúvel, pois $Y$ é OHI. Desta contradição, decorre que $X \backslash Y$ é denso em $X$.

Mostraremos, agora, que $X \backslash Y$ é $n$-resolúvel. Como $X$ é $(n+1)$-resolúvel, existem $D_{1}, \ldots, D_{n+1}$ subconjuntos densos e dois a dois disjuntos de $X$. Para cada $i \in\{1, \ldots, n+1\}$, seja

$$
U_{i}=X \backslash \overline{\left(D_{i} \backslash Y\right)}
$$

Para cada $i \in\{1, \ldots, n\}$, seja

$$
E_{i}=\left[D_{i} \cup\left(U_{i} \cap D_{n+1}\right)\right] \backslash Y .
$$

Afirmamos que os conjuntos $E_{1}, \ldots, E_{n}$ são dois a dois disjuntos e que cada um deles é denso em $X \backslash Y$. De fato, sejam $i, j \in\{1, \ldots, n\}$, com $i \neq j$. Seja $x \in E_{i}$. Então, $x \in D_{i} \backslash Y$ ou $x \in\left(U_{i} \cap D_{n+1}\right) \backslash Y$. Se $x \in D_{i} \backslash Y$, então $x \notin D_{j} \backslash Y$, pois $D_{i} \cap D_{j}=\emptyset$ e, pela mesma razão, $x \notin\left(U_{j} \cap D_{n+1}\right) \backslash Y$. Portanto, $x \notin E_{j}$. Se $x \in\left(U_{i} \cap D_{n+1}\right) \backslash Y$, então $x \notin D_{j} \backslash Y$, pois $D_{n+1} \cap D_{j}=\emptyset$. Mostraremos que $U_{i} \cap U_{j}=\emptyset$ e, portanto, que $x$ não pertence ao conjunto $\left(U_{j} \cap D_{n+1}\right) \backslash Y$. Com efeito, se $U_{i} \cap U_{j} \neq \emptyset$, o conjunto $\left(U_{i} \cap U_{j}\right) \cap Y$ é um aberto não vazio de $Y$. Como $Y$ é OHI, devemos ter que $\left(U_{i} \cap U_{j}\right) \cap Y$ é irresolúvel. Contudo, $D_{i} \cap\left(U_{i} \cap U_{j}\right)$ e $D_{j} \cap\left(U_{i} \cap U_{j}\right)$ são subconjuntos densos e disjuntos de $\left(U_{i} \cap U_{j}\right) \cap Y$, o que mostra que 
$\left(U_{i} \cap U_{j}\right) \cap Y$ é resolúvel. De fato, seja $V=W \cap\left[\left(U_{i} \cap U_{j}\right) \cap Y\right]$ um aberto não vazio de $\left(U_{i} \cap U_{j}\right) \cap Y$, onde $W$ é aberto em $X$. Como $W \cap U_{i} \cap U_{j}$ é um aberto não vazio de $X$, temos que $D_{i} \cap\left(W \cap U_{i} \cap U_{j}\right) \neq \emptyset$ e $D_{j} \cap\left(W \cap U_{i} \cap U_{j}\right) \neq \emptyset$. Como $D_{i} \cap U_{i} \subset Y$ e $D_{j} \cap U_{j} \subset Y$, temos que $D_{i} \cap V \neq \emptyset$ e $D_{j} \cap V \neq \emptyset$. Como $V \subset U_{i} \cap U_{j}$, segue que $\left[D_{i} \cap\left(U_{i} \cap U_{j}\right)\right] \cap V \neq \emptyset$ e $\left[D_{j} \cap\left(U_{i} \cap U_{j}\right)\right] \cap V \neq \emptyset$. Logo, temos que $U_{i} \cap U_{j}=\emptyset$.

Mostremos, finalmente, que $E_{k}$ é denso em $X \backslash Y$, para todo $k \in\{1, \ldots, n\}$. Para tanto, seja $k \in\{1, \ldots, n\}$ e seja $V$ um aberto não vazio de $X \backslash Y$. Temos que $V=$ $W \cap(X \backslash Y)=W \backslash Y$, onde $W$ é um subconjunto aberto de $X$. Se $V \cap\left(D_{k} \backslash Y\right)=\emptyset$, então $(W \backslash Y) \cap\left(D_{k} \backslash Y\right)=\left(W \cap D_{k}\right) \backslash Y=\emptyset$, ou seja, $W \cap D_{k} \subset Y$. Em particular, temos que $W \cap Y$ é um subconjunto aberto não vazio de $Y$.

Se $W \cap D_{n+1} \subset Y$, então $W \cap Y$ é resolúvel, com subconjuntos densos e disjuntos $W \cap D_{k}$ e $W \cap D_{n+1}$. De fato, como $D_{k} \cap D_{n+1}=\emptyset$, temos que $W \cap D_{k}$ e $W \cap D_{n+1}$ são conjuntos disjuntos. Seja $U$ um aberto não vazio de $W \cap Y$. Então, $U=(\Omega \cap W) \cap Y$, onde $\Omega$ é aberto em $X$. Logo, $D_{k} \cap(\Omega \cap W) \neq \emptyset$ e $D_{n+1} \cap(\Omega \cap W) \neq \emptyset$. Como $W \cap D_{k} \subset Y$ e $W \cap D_{n+1} \subset Y$, temos que $\left(D_{k} \cap W\right) \cap U \neq \emptyset$ e $\left(D_{n+1} \cap W\right) \cap U \neq \emptyset$. Logo, $W \cap D_{k}$ e $W \cap D_{n+1}$ são densos em $W \cap Y$. Todavia, isto é um absurdo, já que $Y$ é OHI.

Logo, $\left(W \cap D_{n+1}\right) \backslash Y \neq \emptyset$. Seja $x \in\left(W \cap D_{n+1}\right) \backslash Y$. Então, $x \notin D_{k}$, pois $D_{k} \cap D_{n+1}=\emptyset$ e, portanto, $x \notin D_{k} \backslash Y$.

Se $x \in \overline{D_{k} \backslash Y}$, então $W \cap\left(D_{k} \backslash Y\right)=\left(W \cap D_{k}\right) \backslash Y \neq \emptyset$, o que é absurdo. Assim, $x \notin \overline{D_{k} \backslash Y}$ e, portanto, $x \in U_{k}$. Logo,

$$
\left.\emptyset \neq\left[\left(W \cap D_{n+1}\right) \backslash Y\right] \cap U_{k}=(W \backslash Y) \cap\left[\left(U_{k} \cap D_{n+1}\right) \backslash Y\right]=V \cap\left[\left(U_{k} \cap D_{n+1}\right) \backslash Y\right)\right] .
$$

Portanto, $V \cap E_{k} \neq \emptyset$. 
Lema 4.4. Se X é um espaço topológico tal que

$$
X=Y_{1} \cup \ldots \cup Y_{n}
$$

onde $Y_{1}, \ldots, Y_{n}$ são subespaços $O H I$ e dois a dois disjuntos de $X$, então $X$ não é $(n+1)$ resolúvel.

Demonstração. Seja $n$ o menor número inteiro positivo tal que um espaço topológico $X$ é $(n+1)$-resolúvel e $X=Y_{1} \cup \ldots \cup Y_{n}$, onde $Y_{1}, \ldots, Y_{n}$ são subespaços OHI de $X$, dois a dois disjuntos.

Do lema 4.3 segue que $X \backslash Y_{n}=Y_{1} \cup \ldots \cup Y_{n-1}$ é um espaço topológico $n$-resolúvel, que se escreve como união de $(n-1)$ subespaços OHI, dois a dois disjuntos. Concluímos, portanto, que $n=1$ - o que é absurdo, pois um espaço topológico 2-resolúvel (isto é, resolúvel), não é OHI.

Lema 4.5. Seja $X$ um espaço topológico. Existe um subconjunto aberto $W$ de $X$ (que pode ser vazio ou o próprio $X)$ que contém um subconjunto denso OHI e tal que $X \backslash \bar{W}$ é w-resolúvel na topologia induzida.

Demonstração. Seja $(X, \tau)$ um espaço topológico. Consideremos

$$
\mathcal{U}=\{U \in \tau \backslash\{\emptyset\}: U \text { contém um subconjunto denso OHI }\} \text {. }
$$

Se $\mathcal{U}=\emptyset$, façamos $W=\emptyset$.

Se $\mathcal{U} \neq \emptyset$, seja $\mathcal{A}$ uma família maximal de elementos dois a dois disjuntos de $\mathcal{U}$ e definamos

$$
W=\bigcup_{U \in \mathcal{A}} U
$$


Para cada $U \in \mathcal{A}$, seja $D_{U}$ um subconjunto denso e OHI de $U$. Seja

$$
D_{0}=\bigcup_{U \in \mathcal{A}} D_{U} .
$$

Temos que $D_{0}$ é um subconjunto denso e OHI de $W$.

Mostremos, agora, que todo subconjunto denso de $X \backslash \bar{W}$ é resolúvel. Com efeito, seja $D$ um subconjunto denso de $X \backslash \bar{W}$. Suponhamos, por absurdo, que $D$ não seja resolúvel. Do lema 4.1 concluímos que existe um subconjunto aberto não vazio $U_{1}$ de $D$, que é $\mathrm{OHI}$. Portanto, existe $U_{0}$ aberto em $X$ tal que $U_{1}=D \cap U_{0}$. Podemos supor que $U_{0} \subset X \backslash \bar{W}$ pois $D \subset X \backslash \bar{W}$ e $X \backslash \bar{W}$ é aberto em $X$. Temos que $U_{1}$ é denso em $U_{0}$ e, portanto, $U_{0} \in \mathcal{U}$, o que absurdo.

Provemos, finalmente, que $X \backslash \bar{W}$ é $\omega$-resolúvel. Como $X \backslash \bar{W}$ é denso em $X \backslash \bar{W}$, o mesmo é resolúvel. Logo, existem subconjuntos densos complementares $D_{1}$ e $E_{1}$ de $X \backslash \bar{W}$. Como $E_{1}$ é denso em $X \backslash \bar{W}$, o mesmo também é resolúvel e, portanto, existem subconjuntos densos e complementares $D_{2}$ e $E_{2}$ de $E_{1}$. Como $D_{2}$ e $E_{2}$ são densos em $E_{1}$ e $E_{1}$ é denso em $X \backslash \bar{W}$, segue que $D_{2}$ e $E_{2}$ são densos em $X \backslash \bar{W}$.

Logo, $X \backslash \bar{W}=D_{1} \cup D_{2} \cup E_{2}$. Prosseguindo assim, podemos construir seqüências $\left\{D_{n}\right\}_{n \in \omega}$ e $\left\{E_{n}\right\}_{n \in \omega}$ de subconjuntos densos de $X \backslash \bar{W}$ tais que, para cada $n \in \omega, D_{n+1}$ e $E_{n+1}$ são subconjuntos densos complementares de $E_{n}$. Portanto, $X \backslash \bar{W}$ é $\omega$-resolúvel.

Teorema 4.6. Se X é um espaço topológico n-resolúvel, para todo número natural $n>1$, então X é $\omega$-resolúvel.

Demonstração. Aplicando o lema 4.5, obtemos um subconjunto aberto $W_{1}$ de $X$ e um subconjunto denso $D_{1}$ de $W_{1}$ tais que $D_{1}$ é OHI e $X \backslash \mathrm{cl}_{X}\left(W_{1}\right)$ é $\omega$-resolúvel. Portanto,

$$
X \backslash \mathrm{cl}_{X}\left(W_{1}\right)=E_{1}^{1} \cup E_{2}^{1} \cup \ldots
$$


onde os conjuntos $E_{i}^{1}$ são dois a dois disjuntos e densos em $X \backslash \mathrm{cl}_{X}\left(W_{1}\right)$, para todo $i \in \omega$.

Façamos $X_{0}=X$.

Seja $X_{1}=W_{1} \backslash D_{1}$ e apliquemos, novamente, o lema 4.5. Existem, portanto, um subconjunto aberto $W_{2}$ de $X_{1}$ e um subconjunto denso $D_{2}$ de $W_{2}$ tais que $D_{2}$ é OHI e $X_{1} \backslash \mathrm{cl}_{X_{1}}\left(W_{2}\right)$ é $\omega$-resolúvel.

Prosseguindo desta maneira, encontramos seqüências $\left\{X_{n}\right\}_{n \in \omega},\left\{W_{n}\right\}_{n \in \omega}$ e $\left\{D_{n}\right\}_{n \in \omega}$ tais que $X_{n}=W_{n} \backslash D_{n}, D_{n}$ é um subconjunto denso OHI de $W_{n}, W_{n+1}$ é um subconjunto aberto de $X_{n}$ e $X_{n} \backslash \operatorname{cl}_{X_{n}}\left(W_{n+1}\right)$ é $\omega$-resolúvel.

Assim, para cada $n \in \omega$, temos que

$$
X_{n} \backslash \mathrm{cl}_{X_{n}}\left(W_{n+1}\right)=E_{1}^{n+1} \cup E_{2}^{n+1} \cup \ldots
$$

onde os conjuntos $E_{i}^{n+1}$ são dois a dois disjuntos e densos em $X_{n} \backslash \mathrm{cl}_{X_{n}}\left(W_{n+1}\right)$, para todo $i \in \omega$.

Definamos

$$
F_{1}^{*}=E_{1}^{1} \cup D_{1}
$$

Para cada $n \geq 2$, definamos

$$
F_{n}=E_{n}^{1} \cup \ldots \cup E_{n}^{n} \cup D_{n}
$$

Seja, também,

$$
F_{1}=F_{1}^{*} \cup\left(X \backslash \bigcup_{n \geq 2} F_{n}\right)
$$

Claramente,

$$
X=\bigcup_{n \geq 1} F_{n}
$$

É evidente que $D_{i} \cap D_{j}=\emptyset$, se $i, j \in \omega$, com $i \neq j$. Também é claro que se $m, n \in \omega$ e $m \neq n$, então $E_{i}^{m} \cap E_{j}^{n}=\emptyset$, quaisquer que sejam $i, j \in \omega$. Se $m \geq n$, 
$D_{m} \subset W_{m} \subset W_{n}$ e $E_{k}^{n} \subset X_{n-1} \backslash W_{n}$ e, portanto, $E_{k}^{n} \cap D_{m}=\emptyset$, para todo $k \in \omega$. Se $m<n$, $E_{k}^{n} \subset X_{n-1} \subset X_{m}=W_{m} \backslash D_{m}, \log 0 E_{k}^{n} \cap D_{m}=\emptyset$, para todo $k \in \omega$. Portanto, $F_{i} \cap F_{j}=\emptyset$, se $i, j \in \omega$ e $i \neq j$.

Fixemos $n \in \omega$ arbitrário e mostremos que $X_{n}$ é denso em $W_{n}$. Se isto não ocorresse, existiria um subconjunto aberto $V$ de $X$ tal que

$$
\emptyset \neq V \cap W_{n} \subset W_{n} \backslash X_{n}
$$

Para cada $k=1, \ldots, n$, seja $Z_{k}$ um subconjunto aberto de $X$ tal que $W_{k}=Z_{k} \cap X_{k-1}$. Definamos

$$
U_{0}=V \cap Z_{1} \cap \ldots \cap Z_{n}
$$

Temos que $U_{0}$ é aberto em $X$.

Afirmamos, ainda, que $U_{0} \subset D_{1} \cup \ldots \cup D_{n}$. De fato, se existisse

$$
p \in U_{0} \backslash\left(D_{1} \cup \ldots \cup D_{n}\right)
$$

então, em particular, $p \in Z_{1}=Z_{1} \cap X=Z_{1} \cap X_{0}=W_{1}$. Como $X_{1}=W_{1} \backslash D_{1}$, temos que $p \in X_{1}$ e, portanto, $p \in X_{1} \cap Z_{2}=W_{2}$. Prosseguindo desta maneira, concluímos que $p \in W_{n} . \operatorname{Logo}$,

$$
p \in V \cap W_{n} \subset W_{n} \backslash X_{n}
$$

o que é absurdo, uma vez que $W_{n} \backslash X_{n}=D_{n}$.

Portanto, temos que

$$
U_{0}=U_{0} \cap\left(D_{1} \cup \ldots \cup D_{n}\right)=\left(U_{0} \cap D_{1}\right) \cup \ldots \cup\left(U_{0} \cap D_{n}\right) .
$$

Como $\emptyset \neq V \cap W_{n} \subset W_{n-1} \subset \ldots \subset W_{1}$, segue que $U_{0}$ é um aberto não vazio de $X$. Para cada $k=1, \ldots, n$, temos que $\emptyset \neq V \cap W_{n} \subset U_{0} \cap W_{k}$. Logo, $U_{0} \cap W_{k}$ é um subconjunto aberto não vazio de $W_{k}$. Como $D_{k}$ é denso em $W_{k}$, concluímos que $U_{0} \cap D_{k}$ é um subconjunto aberto não vazio de $D_{k}$. Do fato de $D_{k}$ ser OHI, decorre que $U_{0} \cap D_{k}$ é irresolúvel. 
Como todo aberto não vazio de $U_{0} \cap D_{k}$ é aberto em $D_{k}$, concluímos que $U_{0} \cap D_{k}$ é OHI. Do lema 4.4, segue que $U_{0}$ não é $(n+1)$-resolúvel. Isto implica que $X$ não é $(n+1)$-resolúvel (já que $U_{0}$ é aberto em $X$ ), um absurdo. Portanto, $X_{n}$ é denso em $W_{n}$.

Mostraremos, por fim, que $F_{n}$ é denso em $X$, para todo número natural $n>1$. Para tanto, fixemos um número natural $n>1$ arbitrário e, por absurdo, suponhamos que exista $U$ um subconjunto aberto não vazio de $X$ tal que $F_{n} \cap U=\emptyset$.

Se $U \cap W_{1}=\emptyset$, então $U \cap \mathrm{cl}_{X}\left(W_{1}\right)=\emptyset$ e, portanto, $U \subset X \backslash \mathrm{cl}_{X}\left(W_{1}\right)$.

Se $U \subset X \backslash \operatorname{cl}_{X}\left(W_{1}\right)$, então, como $U$ é aberto em $X \backslash \mathrm{cl}_{X}\left(W_{1}\right)$, temos que

$$
\emptyset \neq U \cap E_{n}^{1} \subset U \cap F_{n}
$$

Portanto, $U \cap W_{1} \neq \emptyset$. Logo, $U \cap X_{1} \neq \emptyset$, já que $X_{1}$ é denso em $W_{1}$.

Se $\emptyset \neq U \cap X_{1} \subset X_{1} \backslash \operatorname{cl}_{X_{1}}\left(W_{2}\right)$, então $\emptyset \neq\left(U \cap X_{1}\right) \cap E_{n}^{2} \subset U \cap F_{n}$. Logo, $U \cap W_{2} \neq \emptyset$ e, portanto, $U \cap X_{2} \neq \emptyset$.

Prosseguindo desta maneira, temos que $U \cap W_{n} \neq \emptyset$. Como $D_{n}$ é denso em $W_{n}$, vale que

$$
\emptyset \neq U \cap W_{n} \cap D_{n} \subset U \cap F_{n}
$$

uma contradição.

Isto prova que $F_{n}$ é denso em $X$, para todo número natural $n>1$. Portanto, $X$ é $\omega$-resolúvel. 


\section{Capítulo 5}

\section{Irresolubilidade no cubo de Cantor $2^{\mathfrak{c}}$}

A primeira seção deste capítulo será dedicada à construção de um subespaço enumerável, denso e submaximal de $2^{\mathrm{c}}$. Com o auxílio de algumas idéias introduzidas em [12] - e que serão analisadas com profundidade no sexto capítulo desta dissertação - elaboramos uma nova demonstração deste resultado, feita diretamente no cubo de Cantor em questão. $\mathrm{Na}$ seção seguinte, mostraremos que $2^{\mathfrak{c}}$ não possui um subespaço denso extremamente desconexo e, portanto, que tal espaço topológico não possui um subespaço denso maximal. As demonstrações do lema 5.2.4 e do teorema 5.2.6 foram extraídas (esta última, com modificações) de [2]. 


\subsection{Um subespaço enumerável, denso e submaximal de $2^{\mathfrak{c}}$}

Definição 5.1.1. Um subconjunto $N$ de um espaço topológico $X$ é dito raro $\operatorname{se} \operatorname{int}(\bar{N})=\emptyset$. Caso contrário, $N$ é dito abundante. A coleção de todos os subconjuntos raros de um espaço topológico $X$ será denotada por $\mathcal{N}(X)$.

Definição 5.1.2. Um espaço topológico $X$ é dito $N O D E C$ se todo subconjunto raro de $X$ for fechado em $X$.

Proposição 5.1.3. Um espaço topológico X é submaximal se, e somente se, é OHI e NODEC.

Demonstração. Seja $X$ um espaço topológico submaximal. Mostremos, primeiramente, que $X$ é OHI. Com efeito, seja $D$ um subconjunto denso qualquer de $X$. Da submaximalidade de $X$, segue que $D$ é aberto em $X$ e, portanto, $\operatorname{int}(D)=D$ é denso em $X$. Do teorema 3.1.2 decorre que $X$ é OHI. Mostremos, agora, que $X$ é NODEC. Para tanto, seja $N$ um subconjunto raro qualquer de $X$. Provemos que $N$ é fechado em $X$ ou, equivalentemente, que $X \backslash N$ é aberto em $X$. Afirmamos que $X \backslash N$ é um subconjunto denso de $X$. De fato, se $U$ é um aberto não vazio de $X$ temos que $U \not \subset \bar{N}$ e, portanto, $U \not \subset N$. Desta forma, $U \cap(X \backslash N) \neq \emptyset$. Utilizando novamente a submaximalidade de $X$, concluímos que $X \backslash N$ é aberto em $X$, o que encerra a demonstração de que $X$ é NODEC.

Reciprocamente, seja $X$ um espaço topológico OHI e NODEC. Afirmamos que $X$ é submaximal. De fato, seja $D$ um subconjunto denso qualquer de $X$. Queremos mostrar que $D$ é aberto em $X$ (ou seja, que $X \backslash D$ é fechado em $X$ ). Como $X$ é NODEC, basta provar que $X \backslash D$ é um subconjunto raro de $X$. Uma vez que $X$ é OHI, do teorema 3.1.2 segue que $\operatorname{int}(D)$ é denso em $X$. Logo, $X \backslash \operatorname{int}(D)$ é um subconjunto fechado de $X$ com 
interior vazio. Como $X \backslash D \subset X \backslash \operatorname{int}(D)$, segue que $\overline{(X \backslash D)} \subset[X \backslash \operatorname{int}(D)]$ e, portanto, $\operatorname{int}[\overline{(X \backslash D)}] \subset \operatorname{int}[(X \backslash \operatorname{int}(D))]=\emptyset$. Daí, concluímos que $X \backslash D$ é um subconjunto raro de $X$, o que encerra a demonstração de que $X$ é submaximal.

Lema 5.1.4. Seja X um espaço topológico. Se todo subconjunto abundante de X contém um aberto não vazio de $X$, então todo subconjunto denso de $X$ tem interior denso em $X$.

Demonstração. Seja $D$ um subconjunto denso de $X$. Suponhamos, por absurdo, que $\operatorname{int}(D)$ não seja denso em $X$. Existe, portanto, um aberto não vazio $U$ de $X$ tal que $\operatorname{int}(D) \cap U=\emptyset$. Como $D$ é denso em $X$, segue que $\overline{U \cap D}=\bar{U}$ e, portanto, $\operatorname{int}(\overline{U \cap D})=\operatorname{int}(\bar{U}) \supset U \neq \emptyset$. Logo, $U \cap D$ é um subconjunto abundante de $X$. Por hipótese, existe $V$ um aberto não vazio de $X$ tal que $V \subset U \cap D$. Portanto, $V \subset D$ e $V \subset U$. Como $V$ é aberto em $X$, segue que $V \subset \operatorname{int}(D)$ e, portanto, $\emptyset \neq V \subset \operatorname{int}(D) \cap U$, uma contradição. Logo, int $(D)$ é denso em $X$.

Exibiremos, a seguir, o resultado mais importante desta seção. A demonstração que apresentamos para o teorema 5.1.5 é inédita e foi obtida através da adaptação de técnicas introduzidas em [12].

Teorema 5.1.5. $O$ cubo de Cantor $2^{\mathfrak{c}}$ possui um subespaço enumerável, denso $e$ submaximal.

Demonstração. Do teorema de Hewitt-Marczewski-Pondiczery, segue que $2^{\mathfrak{c}}$ contém um subespaço denso e enumerável, o qual será denotado por $Y$. Notemos que todo subconjunto 
denso de $2^{\mathfrak{c}}$ é, na verdade, $\omega$-denso no cubo de Cantor em questão, já que $2^{\mathfrak{c}}$ é um espaço topológico $T_{1}$ e todo aberto não vazio de $2^{\mathfrak{c}}$ é infinito.

Seja $\left\{f_{i}\right\}_{i<\omega}$ uma enumeração do conjunto $Y$. Por indução transfinita em $\nu<\mathfrak{c}$ construiremos, a partir de $Y$, um subespaço enumerável e denso do cubo de Cantor $2^{\mathfrak{c}}$ que será, também, OHI e NODEC (e, portanto, submaximal).

Consideremos $\mathfrak{c}=I_{0} \cup I^{\prime}$ uma partição de $\mathfrak{c}, \operatorname{com}\left|I_{0}\right|=\omega$ e $\left|I^{\prime}\right|=\mathfrak{c}$. Particionemos, agora, $I_{0}$ em uma coleção de subconjuntos enumeráveis e não vazios $J_{A, \alpha}$, para todo $A \in[Y]^{<\omega}$ e todo $\alpha \in Y \backslash A$. Note que é possível obter uma tal partição, uma vez que $\left|[Y]^{<\omega}\right|=\omega$.

Por indução transfinita em $\nu<\mathfrak{c}$, definiremos

- Funções parciais finitas $\eta_{\nu} \in \operatorname{Fn}(\mathfrak{c}, 2)$ e subconjuntos

$$
K_{\nu} \subset I^{\prime} \backslash\left[\bigcup_{\zeta<\nu} K_{\zeta} \cup \bigcup\left\{\operatorname{dom} \eta_{\zeta}: \zeta<\nu\right\}\right]
$$

de modo que $K_{\nu}=\emptyset$ ou $\left|K_{\nu}\right|=\omega$.

Note que isto é possível, uma vez que $\left|I^{\prime}\right|=\mathfrak{c},\left|K_{\zeta}\right| \leq \omega$ e $\left|\operatorname{dom} \eta_{\zeta}\right|<\omega$, para todo $\zeta<\nu<\mathfrak{c}$.

- Subespaços $Y_{\nu}=\left\{f_{i}^{\nu}\right\}_{i<\omega}$ enumeráveis e densos no cubo de Cantor $2^{\mathfrak{c}}$, verificando a seguinte propriedade: se $\zeta$ é um ordinal menor do que $\nu$, então

$$
f_{i}^{\nu} \Upsilon_{\left(\mathfrak{c} \backslash I_{\nu}\right) \cup I_{\zeta}}=f_{i}^{\zeta} \Upsilon_{\left(\mathfrak{c} \backslash I_{\nu}\right) \cup I_{\zeta}}
$$

para todo $i<\omega$, onde

$$
I_{\alpha}:=I_{0} \cup\left(\cup_{\beta<\alpha} K_{\beta}\right)
$$

para todo ordinal $\alpha<\mathfrak{c}$. 


$$
\nu=0
$$

Seja $Y_{0}:=\left\{f_{i}^{0}\right\}_{i<\omega}$, onde $f_{i}^{0}(\xi):=f_{i}(\xi)$, se $\xi \in \mathfrak{c} \backslash I_{0}$. Se $\xi \in I_{0}$, existem únicos $A \in[Y]^{<\omega}$ e $\alpha \in Y \backslash A$ tais que $\xi \in J_{A, \alpha}$. Neste caso, façamos

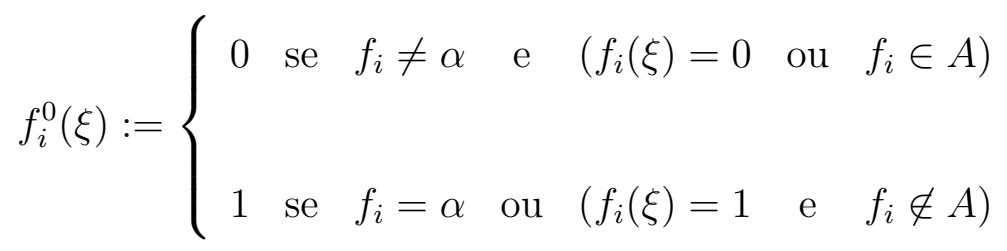

Afirmação 1. $Y_{0}$ é denso no cubo de Cantor $2^{\mathfrak{c}}$.

Um aberto básico do cubo de Cantor $2^{\mathfrak{c}}$ é da forma

$$
\Omega(p)=\prod_{\zeta<\mathfrak{c}} A_{\zeta}
$$

onde $p \in \operatorname{Fn}(\mathfrak{c}, 2) \mathrm{e}$

$$
A_{\zeta}:= \begin{cases}\{p(\zeta)\} & \text { se } \zeta \in \operatorname{dom} p \\ 2 & \text { se } \zeta \notin \operatorname{dom} p\end{cases}
$$

Seja $\Omega(p)$ um aberto básico de $2^{\mathfrak{c}}$. Como $Y$ é denso no cubo de Cantor $2^{\mathfrak{c}}$, segue que $|Y \cap \Omega(p)|=\omega$. Seja $\left\{f_{i}\right\}_{i \in I \subset \omega}$ uma enumeração do conjunto $Y \cap \Omega(p)$.

Consideremos $\left\{f_{i}^{0}\right\}_{i \in I} \subset Y_{0}$. Afirmamos que $\left|\left\{f_{i}^{0}\right\}_{i \in I}\right|=\omega$. De fato, se $\left|\left\{f_{i}^{0}\right\}_{i \in I}\right|<\omega$, existiriam $i, j \in I$, com $i \neq j$, tais que $f_{i}^{0}=f_{j}^{0}$. Desta forma, teremos que $f_{i}=f_{j}$ pois, do contrário, seria possível considerar $A=\left\{f_{i}\right\}$ e $\alpha=f_{j}$, resultando que $f_{i}^{0}(\xi)=0$ e $f_{j}^{0}(\xi)=1$, para todo $\xi \in J_{A, \alpha}$, o que é absurdo, uma vez que $f_{i}^{0}=f_{j}^{0}$. Todavia, isto contradiz o fato de $\left\{f_{i}\right\}_{i \in I}$ ser uma enumeração do conjunto $Y \cap \Omega(p)$. Logo, $\left|\left\{f_{i}^{0}\right\}_{i \in I}\right|=\omega$. 
Se $\operatorname{dom} p \subset \mathfrak{c} \backslash I_{0}$, temos que $\left\{f_{i}^{0}\right\}_{i \in I} \subset Y_{0} \cap \Omega(p)$, pois $f_{i}^{0}(\xi)=f_{i}(\xi)=p(\xi)$, para todo $\xi \in \operatorname{dom} p$. Portanto, $\omega=\left|\left\{f_{i}^{0}\right\}_{i \in I}\right| \leq\left|Y_{0} \cap \Omega(p)\right| \leq \omega$, de onde segue que $\left|Y_{0} \cap \Omega(p)\right|=\omega$.

Se $\operatorname{dom} p \not \subset \mathfrak{c} \backslash I_{0}$, consideremos

$$
\bigcup\left\{A \cup\{\alpha\} \subset Y: J_{A, \alpha} \cap \operatorname{dom} p \neq \emptyset\right\} .
$$

Este é um conjunto finito e não vazio, já que $\left\{J_{A, \alpha}: A \in[Y]^{<\omega}, \alpha \in Y \backslash A\right\}$ constitui uma partição de $I_{0},|\operatorname{dom} p|<\omega$ e $|A|<\omega$. Seja $\left\{f_{i}\right\}_{i \in J \subset \omega}$ uma indexação do conjunto em questão.

Consideremos $\left\{f_{i}^{0}\right\}_{i \in J} \subset Y_{0}$. Claramente, $\left|\left\{f_{i}^{0}\right\}_{i \in J}\right|<\omega$. Mostremos que $\left\{f_{i}^{0}\right\}_{i \in I} \backslash$ $\left\{f_{i}^{0}\right\}_{i \in J} \subset Y_{0} \cap \Omega(p)$.

Para tanto, seja $f_{i}^{0} \in\left\{f_{i}^{0}\right\}_{i \in I} \backslash\left\{f_{i}^{0}\right\}_{i \in J}$, qualquer. Se $\xi \in \operatorname{dom} p \cap\left(\mathfrak{c} \backslash I_{0}\right)$, então $f_{i}^{0}(\xi)=f_{i}(\xi)=p(\xi)$. Se $\xi \in \operatorname{dom} p \cap I_{0}$, então existem únicos $A \in[Y]^{<\omega}$ e $\alpha \in Y \backslash A$ tais que $\xi \in J_{A, \alpha}$. Como $f_{i}^{0} \notin\left\{f_{i}^{0}\right\}_{i \in J}$ (isto é, $i \notin J$ ), temos que $f_{i} \notin A \cup\{\alpha\}$ e, portanto, $f_{i}^{0}(\xi)=f_{i}(\xi)=p(\xi)$.

Logo, $f_{i}^{0} \in Y_{0} \cap \Omega(p)$.

Assim, temos que $\left\{f_{i}^{0}\right\}_{i \in I} \backslash\left\{f_{i}^{0}\right\}_{i \in J} \subset Y_{0} \cap \Omega(p)$. Como $\left|\left\{f_{i}^{0}\right\}_{i \in I}\right|=\omega$ e $\left|\left\{f_{i}^{0}\right\}_{i \in J}\right|<\omega$, segue que $\omega=\left|\left\{f_{i}^{0}\right\}_{i \in I} \backslash\left\{f_{i}^{0}\right\}_{i \in J}\right| \leq\left|Y_{0} \cap \Omega(p)\right| \leq \omega$.

Portanto, $\left|Y_{0} \cap \Omega(p)\right|=\omega$.

Afirmação 2. $\left|Y_{0}\right|=\omega$.

Como $Y_{0}=\left\{f_{i}^{0}\right\}_{i<\omega}$, temos que $\left|Y_{0}\right| \leq \omega$. Da afirmação 1 segue que $\left|Y_{0}\right| \geq \omega$. Logo, $\left|Y_{0}\right|=\omega$

$\nu$ ordinal limite

Seja $Y_{\nu}:=\left\{f_{i}^{\nu}\right\}_{i<\omega}$, onde 


$$
f_{i}^{\nu}(\xi):= \begin{cases}f_{i}(\xi) \quad \text { se } \quad \xi \in \mathfrak{c} \backslash I_{\nu} \\ f_{i}^{0}(\xi) \quad \text { se } \xi \in I_{0} \\ f_{i}^{\zeta^{+}}(\xi) \text { se } \xi \in \cup_{\zeta<\nu} K_{\zeta} \text { onde } \zeta \text { é o único ordinal tal que } \xi \in K_{\zeta}\end{cases}
$$

Note que $\mathfrak{c}=\left(\mathfrak{c} \backslash I_{\nu}\right) \cup I_{0} \cup\left(\cup_{\zeta<\nu} K_{\zeta}\right)$.

Como as hipóteses de indução são válidas para todo $\zeta<\nu$, concluímos que $f_{i}^{\zeta}(\xi)=f_{i}^{0}(\xi)$, para todo $\xi \in\left(\mathfrak{c} \backslash I_{\nu}\right) \cup I_{0}$ (pois, se $\zeta<\nu$, então $I_{\zeta} \subset I_{\nu}$ e, portanto, $\left.\mathfrak{c} \backslash I_{\zeta} \supset \mathfrak{c} \backslash I_{\nu}\right)$.

No caso em que $\xi \in\left(\mathfrak{c} \backslash I_{\nu}\right)$, temos que $f_{i}^{0}(\xi)=f_{i}(\xi)$, já que $I_{0} \subset I_{\nu}$. Logo, $f_{i}^{\nu}(\xi)=f_{i}^{0}(\xi)$, para todo $\xi \in\left(\mathfrak{c} \backslash I_{\nu}\right) \cup I_{0}$ e, portanto, $f_{i}^{\nu}(\xi)=f_{i}^{\zeta}(\xi)$, para todo $\xi \in\left(\mathfrak{c} \backslash I_{\nu}\right) \cup I_{0}$, onde $\zeta<\nu$ é ordinal.

Afirmação 3. $Y_{\nu}$ é denso no cubo de Cantor $2^{\mathfrak{c}}$.

Seja $\Omega(p)$ um aberto básico de $2^{\mathfrak{c}}$. Temos, por hipótese de indução, que $\left|Y_{\zeta} \cap \Omega(p)\right|=\omega$, para todo ordinal $\zeta<\nu$. Mostremos que $\left|Y_{\nu} \cap \Omega(p)\right|=\omega$.

Caso 1: $\operatorname{dom} p \subset\left[\left(\mathfrak{c} \backslash I_{\nu}\right) \cup I_{0}\right]$

Sabemos que $\left|Y_{0} \cap \Omega(p)\right|=\omega$. Seja $\left\{f_{i}^{0}\right\}_{i \in I \subset \omega}$ uma enumeração do conjunto $Y_{0} \cap \Omega(p)$. Consideremos $\left\{f_{i}^{\nu}\right\}_{i \in I} \subset Y_{\nu}$. Neste caso, $f_{i}^{\nu}(\xi)=f_{i}^{0}(\xi)=p(\xi)$, para todo $\xi \in \operatorname{dom} p$ e para todo $i \in I$. Portanto, $\left\{f_{i}^{\nu}\right\}_{i \in I} \subset Y_{\nu} \cap \Omega(p)$. A fim de demonstrar que $\left|\left\{f_{i}^{\nu}\right\}_{i \in I}\right|=\omega$, utilizaremos o lema 4 enunciado abaixo.

Lema 4. Sejam $f_{i}^{0}, f_{j}^{0} \in Y_{0}$ tais que $f_{i}^{0} \neq f_{j}^{0}$. Então, existe $\xi \in I_{0}$ tal que $f_{i}^{0}(\xi) \neq f_{j}^{0}(\xi)$. 
Demonstração. De fato, como $f_{i}^{0} \neq f_{j}^{0}$, temos que $f_{i} \neq f_{j}$. Assim, façamos $A=\left\{f_{i}\right\}$ e $\alpha=f_{j}$. Escolhemos $\xi \in J_{A, \alpha} \subset I_{0}$. Como $f_{j}=\alpha$, segue que $f_{j}^{0}(\xi)=1$. Como $f_{i} \neq \alpha$ e $f_{i} \in A$, segue que $f_{i}^{0}(\xi)=0$.

Suponhamos, por absurdo, que $\left|\left\{f_{i}^{\nu}\right\}_{i \in I}\right|<\omega$. Então, existem $i, j \in I$, com $i \neq j$, tais que $f_{i}^{\nu}=f_{j}^{\nu}$. Em particular, $f_{i}^{\nu}(\xi)=f_{j}^{\nu}(\xi)$, para todo $\xi \in I_{0}$. Mas, se $\xi \in I_{0}$, então $f_{i}^{\nu}(\xi)=f_{i}^{0}(\xi)$ e $f_{j}^{\nu}(\xi)=f_{j}^{0}(\xi)$. Daí, segue que $f_{i}^{0}(\xi)=f_{j}^{0}(\xi)$, para todo $\xi \in I_{0}$. Do lema acima, vem que $f_{i}^{0}=f_{j}^{0}$, o que é absurdo, pois $\left\{f_{i}^{0}\right\}_{i \in I \subset \omega}$ é uma enumeração de $Y_{0} \cap \Omega(p)$.

Logo, $\omega=\left|\left\{f_{i}^{\nu}\right\}_{i \in I}\right| \leq\left|Y_{\nu} \cap \Omega(p)\right| \leq \omega$ e, portanto, $\left|Y_{\nu} \cap \Omega(p)\right|=\omega$.

Caso 2: $\operatorname{dom} p \not \subset\left[\left(\mathfrak{c} \backslash I_{\nu}\right) \cup I_{0}\right]$

Seja

$$
\zeta_{0}:=\max \left\{\zeta<\nu: \operatorname{dom} p \cap K_{\zeta} \neq \emptyset\right\}<\nu
$$

É fácil ver que o conjunto acima descrito é finito e não vazio. Como $\nu$ é ordinal limite, temos que $\zeta_{0}^{+}<\nu$. Além disso, sabemos que $\left|Y_{\zeta_{0}^{+}} \cap \Omega(p)\right|=\omega$.

Seja $\left\{f_{i}^{\zeta_{0}^{+}}\right\}_{i \in I \subset \omega}$ uma enumeração do conjunto $Y_{\zeta_{0}^{+}} \cap \Omega(p)$. Considere $\left\{f_{i}^{\nu}\right\}_{i \in I} \subset Y_{\nu}$. Como $\zeta_{0}^{+}<\nu$ temos, por hipótese de indução, que

$$
f_{i}^{\zeta_{0}^{+}} \Upsilon_{\zeta_{\zeta^{+}}}=f_{i}^{\zeta^{+}} \Upsilon_{\zeta_{\zeta^{+}}}
$$

para todo ordinal $\zeta<\nu$ tal que $\operatorname{dom} p \cap K_{\zeta} \neq \emptyset$, qualquer que seja $i \in I$.

Se $\xi \in \operatorname{dom} p \cap\left[\left(\mathfrak{c} \backslash I_{\nu}\right) \cup I_{0}\right]$, então $f_{i}^{\nu}(\xi)=f_{i}^{\zeta_{0}^{+}}(\xi)=p(\xi)$, para todo $i \in I$.

Se $\xi \in \operatorname{dom} p \cap\left(I_{\nu} \backslash I_{0}\right)=\operatorname{dom} p \cap\left(\cup_{\zeta<\nu} K_{\zeta}\right)$, então $f_{i}^{\nu}(\xi)=f_{i}^{\zeta^{+}}(\xi)=f_{i}^{\zeta_{0}^{+}}(\xi)$, já que $I_{\zeta^{+}} \supset K_{\zeta}$, para todo $i \in I$. Em particular, teremos que

$$
\left\{f_{i}^{\nu}\right\}_{i \in I} \subset Y_{\nu} \cap \Omega(p)
$$


Por um argumento análogo ao utilizado no caso anterior, concluímos que $\left|Y_{\nu} \cap \Omega(p)\right|=\omega$.

Afirmação 5. $\left|Y_{\nu}\right|=\omega$.

Como $Y_{\nu}=\left\{f_{i}^{\nu}\right\}_{i<\omega}$, temos que $\left|Y_{\nu}\right| \leq \omega$. Da afirmação 3, segue que $\left|Y_{\nu}\right| \geq \omega$. Logo, $\left|Y_{\nu}\right|=\omega$.

Afirmação 6. Para todo ordinal $\zeta<\nu$, temos que

$$
f_{i}^{\nu} \Upsilon_{\left(\mathfrak{c} \backslash I_{\nu}\right) \cup I_{\zeta}}=f_{i}^{\zeta} \Upsilon_{\left(\mathfrak{c} \backslash I_{\nu}\right) \cup I_{\zeta}}
$$

para todo $i<\omega$.

Com efeito, fixemos $i<\omega$, qualquer.

Fixemos, também, um ordinal $\zeta<\nu$ arbitrário.

Sabemos que se $\xi \in\left[\left(\mathfrak{c} \backslash I_{\nu}\right) \cup I_{0}\right]$, então $f_{i}^{\nu}(\xi)=f_{i}^{\zeta}(\xi)$.

Se $\xi \in I_{\zeta} \backslash I_{0}=\cup_{\mu<\zeta} K_{\mu}$, então existe um único $\mu<\zeta$ tal que $\xi \in K_{\mu}$. Temos, por definição de $Y_{\nu}$, que $f_{i}^{\nu}(\xi)=f_{i}^{\mu^{+}}(\xi)$. Como $K_{\mu} \subset I_{\mu^{+}}$, segue que $\xi \in I_{\mu^{+}}$. Note que $\mu^{+} \leq \zeta<\nu$ e, portanto, por hipótese de indução, concluímos que $f_{i}^{\zeta}(\xi)=f_{i}^{\mu^{+}}(\xi)$. Logo, $f_{i}^{\nu}(\xi)=f_{i}^{\zeta}(\xi)$. Assim

$$
f_{i}^{\nu}\left\lceil\left(\mathfrak{c} \backslash I_{\nu}\right) \cup I_{\zeta}=f_{i}^{\zeta} \uparrow\left(\mathfrak{c} \backslash I_{\nu}\right) \cup I_{\zeta}\right.
$$

$$
\nu+1
$$

Seja $\mathcal{F}=\left\{F_{\nu}^{0}: \nu<\mathfrak{c}\right\}$ uma indexação dos subconjuntos de $Y_{0}$ que têm cardinalidade $\omega$. Para cada $\nu<\mathfrak{c}$, seja

$$
J_{\nu}=\left\{j<\omega: f_{j}^{0} \in F_{\nu}^{0} \text { e } f_{j}^{0} \neq f_{i}^{0} \text { para todo } i<j \text { tal que } f_{i}^{0} \in F_{\nu}^{0}\right\}
$$


e consideremos $\left\{f_{j}^{0}\right\}_{j \in J_{\nu} \subset \omega}$ uma enumeração do conjunto $F_{\nu}^{0}$.

Definamos, para cada $\nu<\mathfrak{c}, F_{\nu}=\left\{f_{j}^{\nu}\right\}_{j \in J_{\nu}} \subset Y_{\nu}$.

Caso 1: $F_{\nu} \supset \Omega(p) \cap Y_{\nu}$, para algum aberto básico (não vazio) $\Omega(p)$ de $2^{\mathfrak{c}}$

Neste caso, escolhamos alguma $p \in \operatorname{Fn}(\mathfrak{c}, 2)$ tal que $F_{\nu} \supset \Omega(p) \cap Y_{\nu}$ e definamos $\eta_{\nu}:=p$. Definamos, também, $K_{\nu}=\emptyset$.

Façamos $Y_{\nu+1}:=\left\{f_{i}^{\nu+1}\right\}_{i<\omega}$, onde $f_{i}^{\nu+1}:=f_{i}^{\nu}$, para todo $i<\omega$.

Temos, portanto, que $Y_{\nu+1}=Y_{\nu}$ e, desta maneira, todas as hipóteses da indução transfinita em questão estão trivialmente satisfeitas.

Caso 2: $\Omega(p) \cap Y_{\nu} \not \subset F_{\nu}$, para todo aberto básico (não vazio) $\Omega(p)$ de $2^{\mathfrak{c}}$

Neste caso, tomemos $\eta_{\nu}=\emptyset$ e escolhamos

$$
K_{\nu} \subset I^{\prime} \backslash\left[\bigcup_{\zeta<\nu} K_{\zeta} \cup \bigcup\left\{\operatorname{dom} \eta_{\zeta}: \zeta<\nu\right\}\right]
$$

tal que $\left|K_{\nu}\right|=\omega$. Seja $\left\{\xi_{j}: j<\omega\right\}$ uma enumeração de $K_{\nu}$.

Definamos $Y_{\nu+1}:=\left\{f_{i}^{\nu+1}\right\}_{i<\omega}$, onde

$$
f_{i}^{\nu+1}(\xi):=\left\{\begin{array}{lll}
f_{i}(\xi) & \text { se } \quad \xi \in \mathfrak{c} \backslash I_{\nu+1} \\
f_{i}^{0}(\xi) & \text { se } \quad \xi \in I_{0} \\
f_{i}^{\nu}(\xi) & \text { se } \quad \xi \in \cup_{\zeta<\nu} K_{\zeta}
\end{array}\right.
$$

Como $\mathfrak{c}=\left(\mathfrak{c} \backslash I_{\nu+1}\right) \cup I_{0} \cup\left(\cup_{\zeta<\nu} K_{\zeta}\right) \cup K_{\nu}$, resta apenas definir o valor de $f_{i}^{\nu+1}$ nos pontos de $K_{\nu}$, para cada $i \in \omega$.

Se $\xi_{j} \in K_{\nu}$, definamos 


$$
f_{i}^{\nu+1}\left(\xi_{j}\right):=\left\{\begin{array}{lll}
f_{i}^{\nu}\left(\xi_{j}\right) & \text { se } f_{i}^{\nu}=f_{j}^{\nu} \\
f_{i}^{\nu}\left(\xi_{j}\right) & \text { se } f_{i}^{\nu} \neq f_{j}^{\nu} \quad \text { e } \quad f_{i}^{\nu} \notin F_{\nu} \\
1-f_{j}^{\nu}\left(\xi_{j}\right) & \text { se } \quad f_{i}^{\nu} \neq f_{j}^{\nu} \quad \text { e } \quad f_{i}^{\nu} \in F_{\nu}
\end{array}\right.
$$

Sabemos que se $\xi \in\left(\mathfrak{c} \backslash I_{\nu+1}\right) \cup I_{0}$, então $f_{i}^{\nu+1}(\xi)=f_{i}^{0}(\xi)$, uma vez que se $\xi \in \mathfrak{c} \backslash I_{\nu+1}$, então $f_{i}(\xi)=f_{i}^{0}(\xi)$ (pois $\left.I_{0} \subset I_{\nu+1}\right)$.

Como as hipóteses de indução valem para $\nu$, temos que

$$
f_{i}^{\nu}\left\lceil\left(\mathfrak{c} \backslash I_{\nu}\right) \cup I_{0}=f_{i}^{0}\left\lceil\left(\mathfrak{c} \backslash I_{\nu}\right) \cup I_{0}\right.\right.
$$

Logo, se $\xi \in \mathfrak{c} \backslash K_{\nu}$, então $f_{i}^{\nu+1}(\xi)=f_{i}^{\nu}(\xi)$.

Afirmação 7. $Y_{\nu+1}$ é denso no cubo de Cantor $2^{\mathfrak{c}}$.

Suponhamos, por absurdo, que exista $\Omega(p)$ um aberto básico (não vazio) de $2^{\mathfrak{c}}$ tal que $\left|Y_{\nu+1} \cap \Omega(p)\right|<\omega$. Seja $\left\{f_{i}^{\nu+1}\right\}_{i \in I \subset \omega}$ uma indexação do conjunto $Y_{\nu+1} \cap \Omega(p)$.

Afirmamos que existe $\xi^{*} \in I_{0} \backslash \operatorname{dom} p$ tal que $f_{i}^{\nu+1}\left(\xi^{*}\right)=0$, para todo $i \in I$. Com efeito, consideremos $\left\{f_{i}\right\}_{i \in I} \subset Y$. Claramente, $\left|\left\{f_{i}\right\}_{i \in I}\right|<\omega$. Desta maneira, façamos $A=\left\{f_{i}\right\}_{i \in I}$. Tomando $\alpha \in Y \backslash A$ qualquer, segue que, para todo $\xi \in J_{A, \alpha} \subset Y_{0}$, teremos que $f_{i}^{0}(\xi)=0$, para todo $i \in I$. Como $\left\{\alpha \in Y \backslash A: \operatorname{dom} p \cap J_{A, \alpha} \neq \emptyset\right\}$ é finito, para obter $\xi^{*} \in I_{0} \backslash \operatorname{dom} p$ tal que $f_{i}^{0}\left(\xi^{*}\right)=0$, para todo $i \in I$, basta escolher $\alpha \in Y \backslash A$ tal que $\operatorname{dom} p \cap J_{A, \alpha}=\emptyset$, o que é possível, já que $|Y \backslash A|=\omega$. Como $f_{i}^{\nu+1} \uparrow_{I_{0}}=f_{i}^{0} \uparrow_{I_{0}}$, segue a tese.

Seja $q \in \operatorname{Fn}(\mathfrak{c}, 2)$ tal que $\operatorname{dom} q=\operatorname{dom} p \cup\left\{\xi^{*}\right\}, q\left\lceil_{\operatorname{dom} p}=p\right.$ e $q\left(\xi^{*}\right)=1$.

Então, é claro que $Y_{\nu+1} \cap \Omega(q)=\emptyset$. 
Afirmamos que $Y_{\nu+1} \supset Y_{\nu} \backslash F_{\nu}$. De fato, seja $f_{i}^{\nu} \in Y_{\nu} \backslash F_{\nu}$, para algum $i<\omega$, qualquer. Sabemos que $f_{i}^{\nu+1}(\xi)=f_{i}^{\nu}(\xi)$, para todo $\xi \in \mathfrak{c} \backslash K_{\nu}$. Se $\xi_{j} \in K_{\nu}$, então $f_{i}^{\nu+1}\left(\xi_{j}\right)=f_{i}^{\nu}\left(\xi_{j}\right)$, já que $f_{i}^{\nu} \notin F_{\nu}$. Logo, $f_{i}^{\nu}=f_{i}^{\nu+1} \in Y_{\nu+1}$.

Desta forma, $Y_{\nu+1} \supset Y_{\nu} \backslash F_{\nu}$ e, em particular, temos que

$$
Y_{\nu+1} \cap \Omega(q) \supset\left[Y_{\nu} \cap \Omega(q)\right] \backslash F_{\nu} \neq \emptyset
$$

o que é absurdo, posto que $Y_{\nu+1} \cap \Omega(q)=\emptyset$.

Logo, $\left|Y_{\nu+1} \cap \Omega(p)\right| \geq \omega$.

Como $Y_{\nu+1}=\left\{f_{i}^{\nu+1}\right\}_{i<\omega}$, segue que $\left|Y_{\nu+1}\right| \leq \omega$.

Portanto, $\left|Y_{\nu+1} \cap \Omega(p)\right|=\omega$.

Afirmação 8. $\left|Y_{\nu+1}\right|=\omega$.

Como $Y_{\nu+1}=\left\{f_{i}^{\nu+1}\right\}_{i<\omega}$, temos que $\left|Y_{\nu+1}\right| \leq \omega$.

Da afirmação 7 segue que $\left|Y_{\nu+1}\right| \geq \omega$.

Logo, $\left|Y_{\nu+1}\right|=\omega$.

Afirmação 9. Para todo ordinal $\zeta<\nu+1$, temos que

$$
f_{i}^{\nu+1} \Upsilon_{\left(\mathfrak{c} \backslash I_{\nu+1}\right) \cup I_{\zeta}}=f_{i}^{\zeta} \Upsilon_{\left(\mathfrak{c} \backslash I_{\nu+1}\right) \cup I_{\zeta}}
$$

para todo $i<\omega$.

Seja $\zeta<\nu+1$ um ordinal qualquer. Como $K_{\nu} \subset I_{\nu+1}$ e $K_{\nu} \cap I_{\zeta}=\emptyset$, temos que se $\xi \in\left(\mathfrak{c} \backslash I_{\nu+1}\right) \cup I_{\zeta}$, então $f_{i}^{\nu+1}(\xi)=f_{i}^{\nu}(\xi)$. Como as hipóteses de indução valem para $\nu$, segue que $f_{i}^{\nu}(\xi)=f_{i}^{\zeta}(\xi)$, para todo $\xi \in\left(\mathfrak{c} \backslash I_{\nu+1}\right) \cup I_{\zeta}$.

$\operatorname{Logo} f_{i}^{\nu+1}(\xi)=f_{i}^{\zeta}(\xi)$, para todo $\xi \in\left(\mathfrak{c} \backslash I_{\nu+1}\right) \cup I_{\zeta}$.

Seja $Z=Y_{\mathfrak{c}}:=\left\{f_{i}^{\mathfrak{c}}\right\}_{i<\omega}$, onde 


$$
f_{i}^{\mathfrak{c}}(\xi):= \begin{cases}f_{i}(\xi) \quad \text { se } \xi \in \mathfrak{c} \backslash I_{\mathfrak{c}} \\ f_{i}^{0}(\xi) \quad \text { se } \xi \in I_{0} \\ f_{i}^{\zeta^{+}}(\xi) \quad \text { se } \xi \in \cup_{\zeta<\mathfrak{c}} K_{\zeta} \text { onde } \zeta \text { é o único ordinal tal que } \xi \in K_{\zeta}\end{cases}
$$

$\mathrm{e}$

$$
I_{\mathfrak{c}}:=I_{0} \cup\left(\cup_{\zeta<\mathfrak{c}} K_{\zeta}\right)
$$

Por um argumento análogo ao utilizado no passo limite da indução transfinita, concluímos que $Z$ é um subespaço enumerável e denso do cubo de Cantor $2^{\mathfrak{c}}$ tal que se $\zeta<\mathfrak{c}$, então

$$
f_{i}^{\mathfrak{c}}\left\lceil\left(\mathfrak{c} \backslash I_{\mathfrak{c}}\right) \cup I_{\zeta}=f_{i}^{\zeta}\left\lceil\left(\mathfrak{c} \backslash I_{\mathfrak{c}}\right) \cup I_{\zeta}\right.\right.
$$

para todo $i<\omega$.

Além disso, observamos que todo aberto não vazio de $Z$ contém um conjunto da forma $Z \cap \Omega(p)$, para alguma $p \in \operatorname{Fn}(\mathfrak{c}, 2)$. Como $Z$ é denso em $2^{\mathfrak{c}}$, segue que $|Z \cap \Omega(p)|=\omega$ e, portanto, todo aberto não vazio de $Z$ contém, pelo menos, $\omega$ pontos distintos (em outras palavras, $\Delta(Z)=\omega)$.

Afirmação I. Todo subconjunto finito de $Z$ é raro.

Com efeito, como $2^{\mathfrak{c}}$ é um espaço topológico $T_{1}$, segue que $Z$ também o é e, portanto, todo subconjunto finito de $Z$ é fechado. Da observação acima, concluímos que a aderência 
de todo subconjunto finito de $Z$ tem interior vazio em $Z$ e, portanto, todo subconjunto finito de $Z$ é raro.

\section{Afirmação II. $Z$ é NODEC.}

Seja $F$ um subconjunto raro de $Z$. Mostremos que $F$ é fechado em $Z$. Já sabemos que se $F$ é finito, então $F$ é fechado em $Z$. Portanto, basta considerar o caso em que $|F|=\omega$.

Seja $\left\{f_{i}^{\mathfrak{c}}\right\}_{i \in I \subset \omega}$ uma enumeração do conjunto $F$.

Consideremos $\left\{f_{i}^{0}\right\}_{i \in I} \subset Y_{0}$. Temos que, se $i, j \in I$ e $i \neq j$, então $f_{i}^{0} \neq f_{j}^{0}$ (pois, do contrário, teríamos que $f_{i}^{\mathfrak{c}}=f_{j}^{\mathfrak{c}}$, o que é absurdo, uma vez que $\left\{f_{i}^{\mathfrak{c}}\right\}_{i \in I}$ é uma enumeração de $F$ ). Em particular, segue que $\left|\left\{f_{i}^{0}\right\}_{i \in I}\right|=\omega$.

Como $\mathcal{F}$ é a coleção dos subconjuntos de $Y_{0}$ de cardinalidade $\omega$, concluímos que $\left\{f_{i}^{0}\right\}_{i \in I} \in \mathcal{F}$ e, portanto, existe $\nu<\mathfrak{c}$ tal que $F_{\nu}^{0}=\left\{f_{i}^{0}\right\}_{i \in I}$.

Consideremos $F_{\nu}=\left\{f_{i}^{\nu}\right\}_{i \in I} \subset Y_{\nu}$.

Se $F_{\nu} \supset Y_{\nu} \cap \Omega(p)$, para alguma $p \in \operatorname{Fn}(\mathfrak{c}, 2)$, podemos assumir que $F_{\nu} \supset Y_{\nu} \cap \Omega\left(\eta_{\nu}\right)$. Afirmamos que $F \supset Z \cap \Omega\left(\eta_{\nu}\right)$.

Com efeito, seja $f_{i}^{\mathfrak{c}} \in Z \cap \Omega\left(\eta_{\nu}\right)$ qualquer, onde $i<\omega$. Sabemos que $f_{i}^{\nu} \in Y_{\nu}$. Temos também que se $\xi \in \operatorname{dom} \eta_{\nu}$, então $\xi \notin K_{\mu}$, para todo $\mu>\nu$. Lembremos também que, neste passo da indução transfinita, fizemos $K_{\nu}=\emptyset$ e, portanto, $\xi \notin K_{\nu}$. Logo, temos que $\xi \in\left(\mathfrak{c} \backslash \cup_{\nu \leq \mu<\mathfrak{c}} K_{\mu}\right)=\left(\mathfrak{c} \backslash I_{\mathfrak{c}}\right) \cup I_{\nu}$ e, portanto, segue que $f_{i}^{\mathfrak{c}}(\xi)=f_{i}^{\nu}(\xi)$. Assim, como $f_{i}^{\mathfrak{c}} \in \Omega\left(\eta_{\nu}\right)$, temos que se $\xi \in \operatorname{dom} \eta_{\nu}$, então $f_{i}^{\mathfrak{c}}(\xi)=\eta_{\nu}(\xi)$. Logo, $f_{i}^{\nu}(\xi)=\eta_{\nu}(\xi)$, de onde se conclui que $f_{i}^{\nu} \in \Omega\left(\eta_{\nu}\right)$. Como $Y_{\nu} \cap \Omega\left(\eta_{\nu}\right) \subset F_{\nu}$, segue que $f_{i}^{\nu} \in F_{\nu}=\left\{f_{i}^{\nu}\right\}_{i \in I}$ e, portanto, $f_{i}^{\mathfrak{c}} \in F$.

Deste modo, $\bar{F} \supset \overline{Z \cap \Omega\left(\eta_{\nu}\right)}=\overline{\Omega\left(\eta_{\nu}\right)} \supset \Omega\left(\eta_{\nu}\right) \neq \emptyset$, o que é absurdo, pois $F$ é um subconjunto raro de $Z$.

Logo, $F_{\nu} \not \supset Y_{\nu} \cap \Omega(p)$, qualquer que seja $p \in \operatorname{Fn}(\mathfrak{c}, 2)$.

Mostremos, primeiramente, que $\left\{f_{i}^{\nu+1}\right\}_{i \in I}$ é discreto e fechado em $Y_{\nu+1}$. 
Para tanto, seja $f_{i}^{\nu+1} \in\left\{f_{i}^{\nu+1}\right\}_{i \in I}$, qualquer.

Consideremos $p \in \operatorname{Fn}(\mathfrak{c}, 2)$ tal que $\operatorname{dom} p=\left\{\xi_{i}\right\} \subset K_{\nu}$ e $p\left(\xi_{i}\right)=f_{i}^{\nu}\left(\xi_{i}\right)$.

Temos que $f_{i}^{\nu+1}\left(\xi_{i}\right)=f_{i}^{\nu}\left(\xi_{i}\right)=p\left(\xi_{i}\right)$ e, portanto, $f_{i}^{\nu+1} \in\left\{f_{i}^{\nu+1}\right\}_{i \in I} \cap \Omega(p)$. Se $f_{j}^{\nu+1} \in\left\{f_{i}^{\nu+1}\right\}_{i \in I}$ (isto é, se $j \in I$ ) é tal que $f_{j}^{\nu+1} \neq f_{i}^{\nu+1}$, então $f_{j}^{\nu} \in F_{\nu}$ e $f_{j}^{\nu} \neq f_{i}^{\nu}$. Logo, $f_{j}^{\nu+1}\left(\xi_{i}\right)=1-f_{i}^{\nu}\left(\xi_{i}\right) \neq p\left(\xi_{i}\right)$.

Daí, concluímos que $\Omega(p) \cap\left\{f_{i}^{\nu+1}\right\}_{i \in I}=\left\{f_{i}^{\nu+1}\right\}$.

Portanto, $\left\{f_{i}^{\nu+1}\right\}_{i \in I}$ é discreto em $Y_{\nu+1}$.

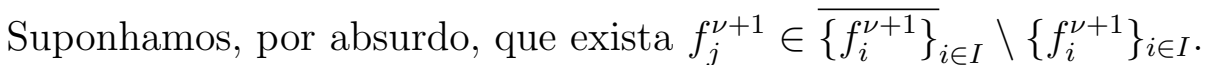

Consideremos $p \in \operatorname{Fn}(\mathfrak{c}, 2)$ tal que $\operatorname{dom} p=\left\{\xi_{j}\right\} \subset K_{\nu}$ e $p\left(\xi_{j}\right)=f_{j}^{\nu}\left(\xi_{j}\right)$.

Afirmamos que $Y_{\nu+1} \cap \Omega(p)$ é um aberto de $Y_{\nu+1}$ ao qual $f_{j}^{\nu+1}$ pertence e que não intersecta $\left\{f_{i}^{\nu+1}\right\}_{i \in I}$. Com efeito, $f_{j}^{\nu+1}\left(\xi_{j}\right)=f_{j}^{\nu}\left(\xi_{j}\right)=p\left(\xi_{j}\right)$ e, portanto, $f_{j}^{\nu+1} \in \Omega(p) \cap Y_{\nu+1}$. Além disso, se $f_{i}^{\nu+1} \in\left\{f_{i}^{\nu+1}\right\}_{i \in I}$ (isto é, se $i \in I$ ), então $f_{i}^{\nu+1}\left(\xi_{j}\right)=1-f_{j}^{\nu}\left(\xi_{j}\right) \neq p\left(\xi_{j}\right)$, pois neste caso $f_{i}^{\nu} \in F_{\nu}$ e $f_{i}^{\nu} \neq f_{j}^{\nu}$. Daí, segue que $Y_{\nu+1} \cap \Omega(p)$ é uma vizinhança aberta de $f_{j}^{\nu+1}$ que não intersecta $\left\{f_{i}^{\nu+1}\right\}_{i \in I}$, o que é absurdo, pois $f_{j}^{\nu+1} \in{\overline{\left\{f_{i}^{\nu+1}\right.}}_{i \in I}$.

Logo, $\left\{f_{i}^{\nu+1}\right\}_{i \in I}$ é fechado em $Y_{\nu+1}$.

Mostremos, agora, que $F=\left\{f_{i}^{\mathfrak{c}}\right\}_{i \in I}$ é discreto e fechado em $Z$.

Para tanto, seja $f_{i}^{\mathfrak{c}} \in F$, qualquer.

Consideremos $p \in \operatorname{Fn}(\mathfrak{c}, 2)$ tal que $\operatorname{dom} p=\left\{\xi_{i}\right\} \subset K_{\nu}$ e $p\left(\xi_{i}\right)=f_{i}^{\nu}\left(\xi_{i}\right)$.

Temos que $F \cap \Omega(p)=\left\{f_{i}^{\mathfrak{c}}\right\}$. De fato, $f_{i}^{\mathfrak{c}}\left(\xi_{i}\right)=f_{i}^{\nu+1}\left(\xi_{i}\right)=f_{i}^{\nu}\left(\xi_{i}\right)=p\left(\xi_{i}\right)$, pois $\xi_{i} \in K_{\nu} \subset I_{\nu+1}$ e $\nu+1<\mathfrak{c}$. Portanto, $f_{i}^{\mathfrak{c}} \in F \cap \Omega(p)$. Além disso, se $f_{j}^{\mathfrak{c}} \in F$ é tal que $f_{j}^{\mathfrak{c}} \neq f_{i}^{\mathfrak{c}}$, então $f_{j}^{\nu} \neq f_{i}^{\nu}$ e, portanto, $f_{j}^{\mathfrak{c}}\left(\xi_{i}\right)=1-f_{i}^{\nu}\left(\xi_{i}\right) \neq p\left(\xi_{i}\right)$. Daí, segue que $f_{j}^{\mathfrak{c}} \notin \Omega(p)$. Logo, $F \cap \Omega(p)=\left\{f_{i}^{\mathfrak{c}}\right\}$.

Suponhamos, por absurdo, que exista $f_{j}^{\mathfrak{c}} \in \bar{F} \backslash F$.

Consideremos $p \in \operatorname{Fn}(\mathfrak{c}, 2)$ tal que $\operatorname{dom} p=\left\{\xi_{j}\right\} \subset K_{\nu}$ e $p\left(\xi_{j}\right)=f_{j}^{\nu}\left(\xi_{j}\right)$.

Afirmamos que $Z \cap \Omega(p)$ é um aberto se $Z$ ao qual $f_{j}^{c}$ pertence e que não intersecta F. Com efeito, $f_{j}^{\mathfrak{c}}\left(\xi_{j}\right)=f_{j}^{\nu+1}\left(\xi_{j}\right)=f_{j}^{\nu}\left(\xi_{j}\right)=p\left(\xi_{j}\right)$, pois $\xi_{j} \in K_{\nu} \subset I_{\nu+1}$ e $\nu+1<\mathfrak{c}$. 
Portanto, $f_{j}^{\mathfrak{c}} \in Z \cap \Omega(p)$. Além disso, se $f_{i}^{\mathfrak{c}} \in F$ (isto é, se $i \in I$ ), então $f_{j}^{\mathfrak{c}}\left(\xi_{j}\right)=f_{j}^{\nu+1}\left(\xi_{j}\right)=$ $1-f_{i}^{\nu}\left(\xi_{j}\right) \neq p\left(\xi_{j}\right)$, pois $f_{i}^{\nu} \in F_{\nu}$ e $f_{i}^{\nu} \neq f_{j}^{\nu}$. Contudo, isto é um absurdo, já que $f_{j}^{c} \in \bar{F}$.

Logo, $F$ é fechado em $Z$.

Portanto, $Z$ é NODEC.

\section{Afirmação III. $Z$ é $O H I$.}

De acordo com o teorema 3.1.2, é suficiente mostrar que todo subconjunto denso de $Z$ tem interior denso em $Z$. Utilizando o lema 5.1.4, basta provar que todo subconjunto abundante de $Z$ contém um aberto não vazio de $Z$.

Para tanto, seja $E$ um subconjunto abundante de $Z$. Já observamos que $|E|=\omega$. Seja $\left\{f_{i}^{\mathfrak{c}}\right\}_{i \in I \subset \omega}$ uma enumeração de $E$.

Consideremos $\left\{f_{i}^{0}\right\}_{i \in I} \subset Y_{0}$. Temos que se $i, j \in I$ e $i \neq j$, então $f_{i}^{0} \neq f_{j}^{0}$ (pois, do contrário, teríamos que $f_{i}^{\mathfrak{c}}=f_{j}^{\mathfrak{c}}$, o que é absurdo, uma vez que $\left\{f_{i}^{\mathfrak{c}}\right\}_{i \in I}$ é uma enumeração de $F)$. Em particular, segue que $\left|\left\{f_{i}^{0}\right\}_{i \in I}\right|=\omega$.

Como $\mathcal{F}$ é a coleção dos subconjuntos de $Y_{0}$ de cardinalidade $\omega$, concluímos que $\left\{f_{i}^{0}\right\}_{i \in I} \in \mathcal{F}$ e, portanto, existe $\nu<\mathfrak{c}$ tal que $\left\{f_{i}^{0}\right\}_{i \in I}=F_{\nu}^{0}$.

Seja $F_{\nu}=\left\{f_{i}^{\nu}\right\}_{i \in I}$.

Se $F_{\nu} \not \supset Y_{\nu} \cap \Omega(p)$, qualquer que seja $p \in \operatorname{Fn}(\mathfrak{c}, 2)$, então $E$ será fechado e discreto em $Z$ (ver demonstração da afirmação II) e, portanto, $E$ não será abundante em $Z$ (de fato, como $\bar{E}=E$, basta mostrar que $\operatorname{int}(E)=\emptyset$. Caso existisse um aberto não vazio $U$ de $Z$ contido em $E$, tomaríamos $p \in U \subset E$. Como $E$ é discreto em $Z$, existe um aberto $V$ de $Z$ tal que $V \cap E=\{p\}$. Logo, $U \cap V=\{p\}$ é um aberto de $Z$, o que significa que $\Delta(Z)=1$, um absurdo).

Desta forma, $F_{\nu} \supset Y_{\nu} \cap \Omega(p)$, para alguma $p \in \operatorname{Fn}(\mathfrak{c}, 2)$. Da demonstração da afirmação II, conclui-se que $E \supset Z \cap \Omega\left(\eta_{\nu}\right)$.

Logo, $Z$ é OHI. 
Das afirmações II e III, concluímos que $Z$ é OHI e NODEC e, portanto, do lema 5.1.3, segue que $Z$ é submaximal.

O resultado que consta no próximo corolário já havia sido demonstrado, por outros métodos, em [2].

Corolário 5.1.6. O cubo de Cantor $2^{\mathfrak{c}}$ possui um subespaço enumerável, denso e irresolúvel.

Demonstração. Segue imediatamente do teorema 5.1.5, uma vez que todo espaço topológico submaximal é, em particular, irresolúvel.

Observamos, por fim, que cada subespaço denso e enumerável de $2^{\mathfrak{c}}$ é homeomeorfo a um subespaço denso de $[0,1]^{\mathfrak{c}}$. De fato, seja $S$ um subconjunto denso e enumerável de $2^{\mathfrak{c}}$. É possível obter uma partição $\left\{I_{\nu}: \nu<\mathfrak{c}\right\}$ de $\mathfrak{c}$ de modo que $\left|I_{\nu}\right|=\omega$ e tal que $2^{I_{\nu}} \backslash\left(S \uparrow_{I_{\nu}}\right)$ é denso em $2^{I_{\nu}}$, qualquer que seja $\nu<\mathfrak{c}$. Para cada $\nu<\mathfrak{c}$, tomemos um subconjunto denso enumerável $D_{\nu} \subset 2^{I_{\nu}} \backslash\left(S \uparrow_{I_{\nu}}\right)$. É conhecido que o espaço topológico $2^{I_{\nu}} \backslash D_{\nu}$ é homeomorfo ao espaço $\mathbb{P}$ dos números irracionais, para todo $\nu<\mathfrak{c}$. Claramente, $S\left\lceil_{I_{\nu}} \subset 2^{I_{\nu}} \backslash D_{\nu} \mathrm{e}\right.$, portanto, $S$ é naturalmente homeomorfo a um subespaço denso de $\prod\left\{2^{I_{\nu}} \backslash D_{\nu}: \nu<\mathfrak{c}\right\}$ que, por sua vez, é homeomorfo ao cubo $\mathbb{P}^{\mathfrak{c}}$. Logo, $S$ é homeomorfo a um subespaço denso de $[0,1]^{c}$. Portanto, do teorema 5.1.5, segue que o cubo de Tychonoff $[0,1]^{\mathfrak{c}}$ também contém um subespaço enumerável, denso e submaximal. 


\subsection{A não existência de um subespaço denso e maximal de $2^{\mathfrak{c}}$}

A fim de mostrar que $2^{\mathfrak{c}}$ não possui um subespaço denso e maximal, provaremos que todo espaço topológico maximal e $T_{2}$ é extremamente desconexo e que o cubo de Cantor em questão não possui um subespaço denso extremamente desconexo.

Definição 5.2.1. Seja $X$ um espaço topológico $T_{2}$. Dizemos que $X$ é extremamente desconexo se o fecho de todo subconjunto aberto de $X$ é, ainda, aberto em $X$.

A fim de justificar o porquê de havermos restringido a definição de espaço topológico extremamente desconexo apenas àqueles que satisfazem o axioma de separação de Hausdorff, consideremos $X$ um conjunto infinito munido da topologia cofinita. Tal espaço topológico é $T_{1}$, mas não é $T_{2}$. Afirmamos que se $U$ é um aberto de $X$, então $\bar{U}$ também o é. Com efeito, se $U=\emptyset$, nada temos a fazer. Suponhamos que $U \neq \emptyset$. Sabemos que $X \backslash U$ é um subconjunto finito de $X$. Como $U \subset \bar{U}$, concluímos que $X \backslash \bar{U} \subset X \backslash U$ e, portanto, $X \backslash \bar{U}$ é também um subconjunto finito de $X$. Logo, $\bar{U}$ é aberto em $X$. Todavia, o espaço topológico em questão é conexo.

Proposição 5.2.2. Todo espaço topológico maximal e $T_{2}$ é extremamente desconexo.

Demonstração. Segue imediatamente do corolário 3.1.10.

Proposição 5.2.3. Um espaço topológico $T_{2}$ é extremamente desconexo se, e somente se, para todo par de abertos disjuntos do espaço em questão vale que suas aderências são, também, disjuntas. 
Demonstração. Seja $X$ um espaço topológico extremamente desconexo. Sejam $U$ e $V$ abertos disjuntos de $X$. Como $U \cap V=\emptyset$ e $V$ é aberto em $X$, segue que $\bar{U} \cap V=\emptyset$. Contudo, $\bar{U}$ é aberto em $X$, já que o espaço em questão é extremamente desconexo. Daí, segue que $\bar{U} \cap \bar{V}=\emptyset$.

Reciprocamente, consideremos $X$ um espaço topológico $T_{2}$ tal que, para todo par de abertos disjuntos de $X$, suas aderências sejam, também, disjuntas. Mostremos que $X$ é extremamente desconexo. Com efeito, tomemos $U$ um aberto qualquer de $X$. Os conjuntos $U$ e $X \backslash \bar{U}$ são abertos disjuntos de $X$ e, portanto, $\bar{U} \cap \overline{(X \backslash \bar{U})}=\emptyset$. Logo,

$$
\bar{U} \subset X \backslash \overline{(X \backslash \bar{U})}=\operatorname{int}(\bar{U})
$$

Daí, segue que $\bar{U}$ é aberto em $X$ e, portanto, $X$ é extremamente desconexo.

Lema 5.2.4. Sejam $Y$ e $Z$ espaços topológicos e seja $f: Y \rightarrow Z$ uma aplicação contínua, sobrejetora e aberta. Se A é um subespaço denso extremamente desconexo de $Y$, então $f(A)$ também é extremamente desconexo.

Demonstração. Por absurdo, suponhamos que $f(A)$ não seja um subespaço extremamente desconexo de $Z$. Assim, existem $z \in f(A)$ e abertos disjuntos $U$ e $V$ de $f(A)$ tais que $z \in \operatorname{cl}_{f(A)}(U) \cap \mathrm{cl}_{f(A)}(V)$. Uma vez que $f(A)$ está munido da topologia induzida por $Z$, existem abertos $U_{1}$ e $V_{1}$ de $Z$ tais que $U_{1} \cap f(A)=U$ e $V_{1} \cap f(A)=V$. Como $A$ é denso em $Y$ e $f$ é uma aplicação contínua e sobrejetora, concluímos que $f(A)$ é denso em $Z$. Logo, $U_{1} \cap V_{1}=\emptyset$.

Afirmamos que $z \in \operatorname{cl}_{Z}\left(U_{1}\right) \cap \mathrm{cl}_{Z}\left(V_{1}\right)$. Com efeito, se $W$ é um aberto de $Z$ ao qual $z$ pertence, então $z \in f(A) \cap W$ e, como $z \in \operatorname{cl}_{f(A)}(U) \cap \operatorname{cl}_{f(A)}(V)$, segue que $[f(A) \cap W] \cap U \neq \emptyset$ e $[f(A) \cap W] \cap V \neq \emptyset$. Como $U \subset U_{1}$ e $V \subset V_{1}$ temos, em particular, que $W \cap U_{1} \neq \emptyset$ e $W \cap V_{1} \neq \emptyset$. Daí, decorre que $z \in \operatorname{cl}_{Z}\left(U_{1}\right) \cap \mathrm{cl}_{Z}\left(V_{1}\right)$. 
Como $z \in f(A)$, existe $y \in A$ tal que $f(y)=z$. Se $\Omega$ é um aberto de $Y$ ao qual $y$ pertence, então $f(\Omega)$ é um aberto de $Z$ ao qual $z$ pertence, já que $f$ é uma aplicação aberta. Portanto, $f(\Omega) \cap U_{1} \neq \emptyset$ e $f(\Omega) \cap V_{1} \neq \emptyset$, o que implica que $\Omega \cap f^{-1}\left(U_{1}\right) \neq \emptyset$ e $\Omega \cap f^{-1}\left(V_{1}\right) \neq \emptyset$.

Consequentemente, temos que

$$
y \in \operatorname{cl}_{Y}\left[f^{-1}\left(U_{1}\right)\right] \cap \operatorname{cl}_{Y}\left[f^{-1}\left(V_{1}\right)\right] \cap A=\operatorname{cl}_{A}\left[f^{-1}\left(U_{1}\right) \cap A\right] \cap \operatorname{cl}_{A}\left[f^{-1}\left(V_{1}\right) \cap A\right] .
$$

Contudo, os conjuntos $U_{2}=f^{-1}\left(U_{1}\right) \cap A$ e $V_{2}=f^{-1}\left(V_{1}\right) \cap A$ são abertos disjuntos de $A$, o que contradiz o fato de $A$ ser extremamente desconexo.

Logo, $f(A)$ é extremamente desconexo.

Lema 5.2.5. Seja $(X, \tau)$ um espaço topológico metrizável. Se $(X, \tau)$ for extremamente desconexo, então $\tau$ é a topologia discreta sobre $X$.

Demonstração. Suponhamos, por absurdo, que $\tau$ não seja a topologia discreta sobre $X$. Existe, portanto, $x \in X$ tal que $\{x\}$ não é aberto em $X$. Como $X$ é metrizável, existe uma seqüência $\left(x_{n}\right)_{n \in \omega}$ de pontos de $X$ que converge para $x$, de modo que $x_{n} \neq x$, para todo $n \in \omega$ e $x_{m} \neq x_{n}$, se $m, n \in \omega$ e $m \neq n$. Sejam

$$
A=\left\{x_{2 n}: n \in \omega\right\}
$$

e

$$
B=\left\{x_{2 n+1}: n \in \omega\right\} .
$$

Consideremos

$$
U=\{y \in X \backslash\{x\}: d(y, A)<d(y, B)\}
$$


e

$$
V=\{y \in X \backslash\{x\}: d(y, A)>d(y, B)\}
$$

Temos que $U$ e $V$ são abertos infinitos e disjuntos de $X$.

Como $x \in \bar{U} \cap \bar{V}$, da proposição 5.2.3 segue que $X$ não é extremamente desconexo, uma contradição.

Teorema 5.2.6. O cubo de Cantor $2^{\mathfrak{c}}$ não possui um subespaço denso extremamente desconexo.

Demonstração. Suponhamos, por absurdo, que $A$ seja um subespaço denso extremamente desconexo de $2^{\mathfrak{c}}$. Temos que a projeção

$$
\Pi: 2^{\mathfrak{c}} \rightarrow 2^{\omega}
$$

é uma aplicação contínua, aberta e sobrejetora.

Do lema 5.2.4, segue que $2^{\omega}$ contém um subespaço denso extremamente desconexo, o que é absurdo, pois todo subespaço denso de $2^{\omega}$ é metrizável e não possui pontos isolados.

Corolário 5.2.7. O cubo de Cantor $2^{\mathfrak{c}}$ não possui um subespaço denso maximal.

Demonstração. Observamos que todo subespaço de $2^{\mathfrak{c}}$ é $T_{2}$, pois o cubo de Cantor em questão também o é. Portanto, a afirmação presente no enunciado deste corolário decorre diretamente da proposição 5.2.2 e do teorema 5.2.6. 


\section{Capítulo 6}

\section{Espaços $\mathcal{D}$-forçados}

Na primeira seção deste capítulo, faremos uma pequena introdução à teoria dos espaços topológicos $\mathcal{D}$-forçados, exibindo algumas propriedades importantes dos mesmos e que serão utilizadas adiante. A segunda seção será dedicada ao principal teorema deste capítulo, o qual é um resultado técnico e de extrema importância para a seção subseqüente. Na terceira e última seção, apresentaremos várias aplicações interessantes dos resultados obtidos na seção anterior. Demonstraremos que, para todo cardinal infinito $\kappa$, o cubo de Cantor $2^{2^{\kappa}}$ possui um subespaço denso e submaximal de cardinalidade $\kappa$. Provaremos, ainda, que dado um cardinal infinito $\mu$, existe um espaço topológico completamente regular que é $\mu$-resolúvel, mas que não é $\mu^{+}$-resolúvel. Mostraremos, por fim, que se $\lambda$ é um cardinal regular não enumerável, é possível exibir um exemplo de espaço topológico que é $\mu$-resolúvel, para todo $\mu<\lambda$, mas que não é $\lambda$-resolúvel. Os principais resultados apresentados neste capítulo foram extraídos de [12]. 


\subsection{Introdução aos espaços $\mathcal{D}$-forçados}

Sejam $X$ um espaço topológico e $\mathcal{D}$ uma família de subconjuntos densos de $X$.

Definição 6.1.1. Um subconjunto $M$ de $X$ é dito um $(\mathcal{D}, X)$-mosaico se existe uma família celular maximal $\mathcal{V}$ de $X$ tal que, para cada $V \in \mathcal{V}$, é possível obter $D_{V} \in \mathcal{D}$ de modo que

$$
M=\bigcup\left\{V \cap D_{V}: V \in \mathcal{V}\right\}
$$

Definição 6.1.2. Um subconjunto $M$ de $X$ é dito um $(\mathcal{D}, X)$-mosaico parcial se existe uma família celular $\mathcal{V}$ de $X$ tal que, para cada $V \in \mathcal{V}$, é possível obter $D_{V} \in \mathcal{D}$ de modo que

$$
M=\bigcup\left\{V \cap D_{V}: V \in \mathcal{V}\right\}
$$

Definição 6.1.3. Um subconjunto $P$ de $X$ da forma

$$
P=D \cap U
$$

onde $D \in \mathcal{D}$ e $U$ é um aberto não vazio de $X$ é dito um $(\mathcal{D}, X)$-pedaço.

Quando não houver dúvidas acerca do espaço topológico $X$ considerado, escreveremos $\mathcal{D}$-mosaico, em vez de $(\mathcal{D}, X)$-mosaico, $\mathcal{D}$-mosaico parcial, em vez de $(\mathcal{D}, X)$-mosaico parcial e $\mathcal{D}$-pedaço, em vez de $(\mathcal{D}, X)$-pedaço.

Proposição 6.1.4. Sejam $X$ um espaço topológico e $\mathcal{D}$ uma família de subconjuntos densos de $X$. Todo $\mathcal{D}$-mosaico é denso em $X$ e todo $\mathcal{D}$-pedaço é abundante em $X$.

Demonstração. Seja

$$
M=\bigcup\left\{V \cap D_{V}: V \in \mathcal{V}\right\}
$$


um $\mathcal{D}$-mosaico, onde $\mathcal{V}$ é uma família celular maximal de $X$. Se $M$ não fosse denso em $X$, existiria um subconjunto aberto não vazio $U$ de $X$ tal que $U \cap M=\emptyset$. Como $D_{V} \in \mathcal{D}$, para todo $V \in \mathcal{V}$, concluímos que $\mathcal{V} \cup\{U\}$ é uma família celular de $X$ que contém $\mathcal{V}$ propriamente, o que contraria a maximalidade de $\mathcal{V}$. Logo, $M$ é um subconjunto denso de $X$.

Seja

$$
P=D \cap U
$$

um $\mathcal{D}$-pedaço, onde $D \in \mathcal{D}$ e $U$ é um aberto não vazio de $X$. Temos que $\bar{P}=\overline{D \cap U}=\bar{U}$ e, portanto,

$$
\operatorname{int}(\bar{P})=\operatorname{int}(\bar{U}) \supset U \neq \emptyset
$$

Logo, $P$ é um subconjunto abundante de $X$.

Apresentaremos, a seguir, a principal definição deste capítulo.

Definição 6.1.5. Seja $\mathcal{D}$ uma família de subconjuntos densos de um espaço topológico $X$. Dizemos que $X$ é $\mathcal{D}$-forçado se todo subconjunto denso de $X$ contém um $\mathcal{D}$-mosaico.

A próxima proposição apresenta uma caracterização alternativa dos espaços topológicos $\mathcal{D}$-forçados.

Proposição 6.1.6. Um espaço topológico $X$ é $\mathcal{D}$-forçado se, e somente se, todo subconjunto abundante de $X$ contém um $\mathcal{D}$-pedaço.

Demonstração. Suponhamos que $X$ seja um espaço topológico $\mathcal{D}$-forçado. Seja $A$ um subconjunto abundante de $X$. Consideremos

$$
S=A \cup(X \backslash \bar{A})
$$


Temos que

$$
\bar{S} \supset \bar{A} \cup(X \backslash \bar{A})=X
$$

Portanto, $S$ é denso em $X$. Como $X$ é $\mathcal{D}$-forçado, $S$ contém um $\mathcal{D}$-mosaico

$$
M=\bigcup\left\{V \cap D_{V}: V \in \mathcal{V}\right\}
$$

onde $\mathcal{V}$ é uma família celular maximal de $X$ e $D_{V} \in \mathcal{D}$, para todo $V \in \mathcal{V}$.

Como $A$ é abundante em $X$, existe um subconjunto aberto não vazio $U$ de $X$ tal que $U \subset \bar{A}$. Portanto, $U \cap(X \backslash \bar{A})=\emptyset$.

Se $U \in \mathcal{V}$, então $U \cap D_{U} \subset M \subset S$. Como $U \cap D_{U} \subset U \subset \bar{A}$, temos que $\left(U \cap D_{U}\right) \cap(X \backslash \bar{A})=\emptyset$. Portanto, o $\mathcal{D}$-pedaço $U \cap D_{U}$ está contido em $A$. Se $U \notin \mathcal{V}$, da maximalidade de $\mathcal{V}$ segue que existe $V \in \mathcal{V}$ tal que $U \cap V \neq \emptyset$. Logo, $D_{V} \cap(U \cap V) \neq \emptyset$, pois $D_{V}$ é denso em $X$. Como $V \cap D_{V} \subset M \subset S$, temos que $D_{V} \cap(U \cap V) \subset S$. Como $D_{V} \cap(U \cap V) \subset U \subset \bar{A}$, segue que $\left[D_{V} \cap(U \cap V)\right] \cap(X \backslash \bar{A})=\emptyset$. Portanto, o $\mathcal{D}$-pedaço $D_{V} \cap(U \cap V)$ está contido em $A$.

Reciprocamente, seja $X$ um espaço topológico tal que todo subconjunto abundante de $X$ contém um $\mathcal{D}$-pedaço. Mostremos que $X$ é $\mathcal{D}$-forçado. Para tanto, seja $D$ um subconjunto denso de $X$ e seja

$$
\mathcal{A}=\left\{Y \subset D: \operatorname{int}_{X}\left[\mathrm{cl}_{X}(Y)\right] \neq \emptyset\right\}
$$

Notemos que $\mathcal{A} \neq \emptyset$, pois $D \in \mathcal{A}$.

Por hipótese, cada $Y \in \mathcal{A}$ contém um $\mathcal{D}$-pedaço

$$
P_{Y}=V_{Y} \cap D_{V_{Y}}
$$

onde $V_{Y}$ é um aberto não vazio de $X$ e $D_{V_{Y}}$ é um elemento de $\mathcal{D}$.

Seja $\mathcal{V}$ uma família maximal de elementos dois a dois disjuntos do conjunto $\left\{V_{Y}: Y \in\right.$ $\mathcal{A}$ \}. Afirmamos que todo aberto não vazio de $X$ intersecta algum elemento de $\mathcal{V}$. De fato, 
seja $U$ um aberto não vazio de $X$. Como $D$ é um subconjunto denso de $X$, temos que $\overline{U \cap D}=\bar{U}$ e, portanto, $\operatorname{int}(\overline{U \cap D})=\operatorname{int}(\bar{U}) \supset U \neq \emptyset$. Logo, $U \cap D \in \mathcal{A}$. Fazendo $Y=U \cap D$, concluímos que existe um $\mathcal{D}$-pedaço

$$
P_{Y}=V_{Y} \cap D_{V_{Y}}
$$

onde $V_{Y} \in \mathcal{A}$ é um aberto não vazio de $X$ e $D_{V_{Y}}$ é um elemento $\mathcal{D}$, tal que $P_{Y} \subset Y$.

Se $V_{Y} \in \mathcal{V}$, nada temos a fazer. Se $V_{Y} \notin \mathcal{V}$, em virtude da maximalidade de $\mathcal{V}$, existe $V_{\widetilde{Y}} \in \mathcal{V}$ tal que $V_{Y} \cap V_{\widetilde{Y}} \neq \emptyset$. Como $D_{V_{Y}}$ é denso em $X$, segue que $V_{Y} \cap V_{\widetilde{Y}} \cap D_{V_{Y}} \neq \emptyset$. Logo, $\emptyset \neq V_{\tilde{Y}} \cap P_{Y} \subset U \cap V_{\tilde{Y}}$.

Portanto, $\mathcal{V}$ é uma família celular maximal de $X$. Seja

$$
M=\bigcup\left\{V_{Y} \cap D_{V_{Y}}: V_{Y} \in \mathcal{V}\right\} .
$$

Temos que $M$ é um $\mathcal{D}$-mosaico e $M \subset D$.

Uma vez que um espaço topológico $X$ é sempre denso em $X$, a escolha mais simples para $\mathcal{D}$ é $\mathcal{D}=\{X\}$.

Proposição 6.1.7. Seja $X$ um espaço topológico. Um subconjunto $P$ de $X$ é um $\{X\}$ pedaço se, e somente se, $P$ é um aberto não vazio de $X$. Um subconjunto $M$ de $X$ é um $\{X\}$-mosaico se, e somente se, $M$ é um aberto denso de $X$. Conseqüentemente, $X$ é $\{X\}$-forçado se, e somente se, é OHI.

Demonstração. Sejam $X$ um espaço topológico e $P$ um subconjunto de $X$. Suponhamos, primeiramente, que $P$ seja um $\{X\}$-pedaço ou seja, que $P=U \cap X=U$, para algum aberto não vazio $U$ de $X$. Então, $P$ é um aberto não vazio de $X$. Reciprocamente, se $P$ é um aberto não vazio de $X$, então $P$ é um $\{X\}$-pedaço, pois $P=P \cap X$. 
Seja $M$ um subconjunto de $X$. Se $M$ é um $\{X\}$-mosaico, então existe $\mathcal{V}$ uma família celular maximal de $X$ tal que

$$
M=\bigcup\{V \cap X: V \in \mathcal{V}\}=\bigcup_{V \in \mathcal{V}} V
$$

Claramente, $M$ é aberto em $X$. Se $M$ não fosse denso em $X$, existiria $U$ um aberto não vazio de $X$ tal que $M \cap U=\emptyset$ e, portanto, $V \cap U=\emptyset$, para todo $V \in \mathcal{V}$. Assim, $\mathcal{V} \cup\{U\}$ seria uma família celular de $X$ que contém $\mathcal{V}$ propriamente, o que contraria a maximalidade de $\mathcal{V}$. Portanto, $M$ é denso em $X$. Reciprocamente, seja $M$ um subconjunto aberto e denso de $X$. Temos que $\{M\}$ é uma família celular maximal de $X$ e $M=M \cap X$. Portanto, $M$ é um $\{X\}$-mosaico.

Se $X$ é um espaço topológico $\{X\}$-forçado, então todo subconjunto denso de $X$ contém um $\{X\}$-mosaico, ou seja, todo subconjunto denso de $X$ contém um aberto denso em $X$. Do teorema 3.1.2 segue que $X$ é OHI. Reciprocamente, se $X$ é OHI, do teorema 3.1.2 concluímos que todo subconjunto denso de $X$ contém um aberto denso em $X$ ou seja, todo subconjunto denso de $X$ contém um $\{X\}$-mosaico. Logo, $X$ é $\{X\}$-forçado.

O próximo resultado fornece uma descrição útil dos subconjuntos raros de espaço topológico $\mathcal{D}$-forçado.

Inicialmente, observamos que um subconjunto $N$ de um espaço topológico $X$ é raro se, e somente se, $S \backslash N$ é denso em $X$, para todo subconjunto denso $S$ de $X$. De fato, seja $N$ um subconjunto raro de um espaço topológico $X$ e suponhamos, por absurdo, que $S \backslash N$ não seja denso em $X$, para algum $S \subset X$ denso. Existe, portanto, um aberto não vazio $U$ de $X$ tal que $(S \backslash N) \cap U=\emptyset$, ou seja, tal que $(S \cap U) \backslash N=\emptyset$. Logo, $S \cap U \subset N$ e daí decorre que $\overline{S \cap U} \subset \bar{N}$. Como $S$ é denso em $X$, temos que $\overline{S \cap U}=\bar{U}$ e, portanto, $\emptyset \neq U \subset \bar{U} \subset \bar{N}$, o que contradiz o fato de $N$ ser raro. 
Reciprocamente, seja $N$ um subconjunto de $X$ tal que $S \backslash N$ é denso em $X$, para todo subconjunto denso $S$ de $X$. Afirmamos, primeiramente, que $(X \backslash \bar{N}) \cup N$ é denso em $X$. De fato, se $U$ é um aberto não vazio de $X$ tal que $U \cap N=\emptyset$, então $U \cap \bar{N}=\emptyset$ e, portanto, $U \subset X \backslash \bar{N}$. Tomando $S=(X \backslash \bar{N}) \cup N$ temos, por hipótese, que $S \backslash N=X \backslash \bar{N}$ é denso em $X$. Daí, segue que $\bar{N}$ não contém nenhum aberto não vazio de $X$ e, portanto, $N$ é raro em $X$.

Lema 6.1.8. Seja X um espaço topológico $\mathcal{D}$-forçado. Então

$$
\mathcal{N}(X)=\{Y \subset X: D \backslash Y \text { é denso em } X \text {, para todo } D \in \mathcal{D}\}
$$

Demonstração. Em virtude da observação acima, é evidente que

$$
\mathcal{N}(X) \subset\{Y \subset X: D \backslash Y \text { é denso em } X \text {, para todo } D \in \mathcal{D}\}
$$

A fim de mostrar a inclusão contrária, tomemos $Y$ um subconjunto de $X$ tal que $D \backslash Y$ é denso em $X$, para todo $D \in \mathcal{D}$ e mostremos que $Y$ é um subconjunto raro de $X$. Com efeito, se $Y$ fosse um subconjunto abundante de $X$, da proposição 6.1 .6 seguiria que $Y$ contém um subconjunto da forma $U \cap D$, onde $D \in \mathcal{D}$ e $U$ é um aberto não vazio de $X$, já que $X$ é, por hipótese, um espaço topológico $\mathcal{D}$-forçado. Então, $(D \backslash Y) \cap U=\emptyset$, ou seja, $D \backslash Y$ não é denso em $X$, o que é absurdo. Logo, $Y$ é um subconjunto raro de $X$.

Lema 6.1.9. Seja $X$ um espaço topológico tal que se $D$ é um subconjunto denso de $X$, então $X \backslash D$ é raro. Nestas condições, $X$ é OHI.

Demonstração. Mostraremos que todo subconjunto denso de $X$ tem interior denso em $X$. Do teorema 3.1.2 seguirá que $X$ é OHI. De fato, seja $D$ um subconjunto denso de $X$. Temos que $\operatorname{int}(D)=X \backslash \overline{(X \backslash D)}$. Seja $U$ um aberto não vazio de $X$. Se $U \cap \operatorname{int}(D)=\emptyset$, 
então $U \subset \overline{X \backslash D}$, o que é absurdo, pois por hipótese $X \backslash D$ é raro. $\operatorname{Logo}, \operatorname{int}(D) \cap U \neq \emptyset$. Portanto, int $(D)$ é denso em $X$.

Proposição 6.1.10. Seja $X$ um espaço topológico $\mathcal{D}$-forçado e considere $S$ um subconjunto denso de $X$ tal que, para cada $D \in \mathcal{D}$, vale que $S \cap D$ é raro ou $S \backslash D$ é raro. Nestas condições, Sé OHI.

Demonstração. Mostraremos que todo subconjunto denso $T$ de $S$ tem complemento raro em $S$. Do lema 6.1.9 decorrerá que $S$ é OHI.

Seja $T$ um subconjunto denso de $S$. Como $S$ é denso em $X, T$ também é denso em $X$. Como $X$ é $\mathcal{D}$-forçado, $T$ contém um $\mathcal{D}$-mosaico

$$
M=\bigcup\left\{V \cap D_{V}: V \in \mathcal{V}\right\}
$$

onde $\mathcal{V}$ é uma família celular maximal de $X$ e $D_{V} \in \mathcal{D}$, para todo $V \in \mathcal{V}$.

Para cada $V \in \mathcal{V}$ temos, por hipótese, que $S \cap D_{V}$ é raro ou que $S \backslash D_{V}$ é raro. Como $M \subset T \subset S$, segue que $V \cap D_{V} \subset S$ e, portanto, $V \cap D_{V} \subset S \cap D_{V}$, para todo $V \in \mathcal{V}$. Mas $\emptyset \neq V \subset \bar{V}=\overline{V \cap D_{V}} \subset \overline{S \cap D_{V}}$. Logo, $S \cap D_{V}$ é abundante, qualquer que seja $V \in \mathcal{V}$ e, portanto, $S \backslash D_{V}$ é raro, para todo $V \in \mathcal{V}$.

Como $(S \cap V) \backslash\left(V \cap D_{V}\right) \subset S \backslash D_{V}$, segue que $(S \cap V) \backslash\left(V \cap D_{V}\right)$ é raro, para todo $V \in \mathcal{V}$. Temos, ainda, que $V \cap D_{V} \subset T \cap V$, para todo $V \in \mathcal{V}$. Portanto, $(S \cap V) \backslash(T \cap V)$ é raro, para todo $V \in \mathcal{V}$. Se $S \backslash T$ não for raro, existirá um aberto não vazio $U$ de $X$ tal que $U \subset \overline{S \backslash T}$. Como $\mathcal{V}$ é uma família celular maximal de $X$, existe $V \in \mathcal{V}$ tal que $V \cap U \neq \emptyset$. Logo, $\emptyset \neq U \cap V \subset \overline{(S \backslash T)} \cap V \subset \overline{[(S \backslash T) \cap V]}$, o que é absurdo, pois $(S \backslash T) \cap V=(S \cap V) \backslash T$ é raro. 
Proposição 6.1.11. Sejam $X$ um espaço topológico e $\mu$ um cardinal tal que $\mu \geq \hat{c}(X)$ - ou seja, tal que $X$ não possua $\mu$ subconjuntos abertos, dois a dois disjuntos. Seja $\mathcal{D}$ uma família de subconjuntos densos de $X$. Suponhamos que para cada $\mathcal{E} \in[\mathcal{D}]^{\mu}$, exista $\mathcal{F} \in[\mathcal{E}]^{\widehat{c}(X)}$ tal que $D \cap D^{\prime}$ é denso em $X$, quando $\left\{D, D^{\prime}\right\} \in[\mathcal{F}]^{2}$. Então, para uma família qualquer de $\mathcal{D}$-pedaços $\left\{P_{i}: i<\mu\right\}$, existe $\{i, j\} \in[\mu]^{2}$ tal que $P_{i} \cap P_{j}$ é abundante em $X$. Em particular, se $X$ é $\mathcal{D}$-forçado e $|\mathcal{D}|^{+} \geq \hat{c}(X)$, então $X$ não é $|\mathcal{D}|^{+}$-resolúvel.

Demonstração. Seja $\left\{P_{i}: i<\mu\right\}$ uma família qualquer de $(\mathcal{D}, X)$-pedaços. Para cada $i<\mu$, existem $U_{i}$ um aberto não vazio de $X$ e $D_{i} \in \mathcal{D}$, com

$$
P_{i}=U_{i} \cap D_{i}
$$

Por hipótese, existe $I \in[\mu]^{\hat{c}(X)}$ tal que $D_{i} \cap D_{j}$ é denso em $X$, para todo $\{i, j\} \in[I]^{2}$. Da definição de $\hat{c}(X)$, decorre que existe $\{i, j\} \in[I]^{2}$ tal que

$$
U_{i} \cap U_{j} \neq \emptyset
$$

Como $D_{i} \cap D_{j}$ é denso em $X$, temos que $\left(D_{i} \cap D_{j}\right) \cap\left(U_{i} \cap U_{j}\right) \neq \emptyset$. Mas

$$
\left(U_{i} \cap U_{j}\right) \cap\left(D_{i} \cap D_{j}\right)=\left(U_{i} \cap D_{i}\right) \cap\left(U_{j} \cap D_{j}\right)=P_{i} \cap P_{j}
$$

Logo,

$$
\emptyset \neq\left(U_{i} \cap U_{j}\right) \subset \overline{\left(U_{i} \cap U_{j}\right)}=\overline{\left(U_{i} \cap U_{j}\right) \cap\left(D_{i} \cap D_{j}\right)}=\overline{P_{i} \cap P_{j}} .
$$

Portanto, $P_{i} \cap P_{j}$ é abundante em $X$.

Suponhamos, agora, que $X$ seja $\mathcal{D}$-forçado e que $|\mathcal{D}|^{+} \geq \hat{c}(X)$. Observamos que todas as hipóteses do teorema estão trivialmente verificadas. Como $X$ é $\mathcal{D}$-forçado, todo subconjunto denso de $X$ contém um $\mathcal{D}$-mosaico e, em particular, um $\mathcal{D}$-pedaço. Portanto, $X$ não é $|\mathcal{D}|^{+}$resolúvel. 
Lema 6.1.12. Seja $\mu>1$ um cardinal. Sejam $\mathcal{D}$ uma família de subconjuntos densos de um espaço topológico $X$ e

$$
M=\bigcup\left\{V \cap D_{V}: V \in \mathcal{V}\right\}
$$

$u m \mathcal{D}$-mosaico parcial, onde $\mathcal{V}$ é uma família celular de $X$. Se $D_{V}$ é $\mu$-resolúvel, para todo $V \in \mathcal{V}$, então $M$ também é $\mu$-resolúvel.

Demonstração. Como $D_{V} \cap V$ é aberto em $D_{V}$, do fato 2.2.3 segue que $D_{V} \cap V$ também é $\mu$-resolúvel. Do fato 2.2.5 decorre que

$$
M=\bigcup\left\{V \cap D_{V}: V \in \mathcal{V}\right\}
$$

também o será.

Definição 6.1.13. Seja $\kappa>1$ um cardinal. Um espaço topológico $X$ é dito hereditariamente $\kappa$-resolúvel se todo subespaço denso em si mesmo de $X$ for $\kappa$-resolúvel.

Proposição 6.1.14. Seja $X$ um espaço topológico $\mathcal{D}$-forçado, onde $\mathcal{D}$ é uma família de subconjuntos densos de $X$. Suponhamos que todo subespaço denso em si mesmo de $X$ é abundante. Então, para cada subespaço denso em si mesmo $S$ de $X$, existe um $\mathcal{D}$-mosaico parcial $M \subset S$ que é denso em $S$. Além disso, se todo $D \in \mathcal{D}$ for $\mu$-resolúvel, para algum cardinal $\mu>1$, então $X$ será hereditariamente $\mu$-resolúvel.

Demonstração. Seja $S$ um subconjunto denso em si mesmo de $X$. Seja $\mathcal{V}$ uma família maximal de subconjuntos abertos de $X$, dois a dois disjuntos, tal que, para cada $V \in \mathcal{V}$, exista $D_{V} \in \mathcal{D}$ com $V \cap D_{V} \subset S$.

Seja

$$
M=\bigcup\left\{V \cap D_{V}: V \in \mathcal{V}\right\}
$$


Temos que $M$ é um $\mathcal{D}$-mosaico parcial e que $M \subset S$. Afirmamos, ainda, que $M$ é denso em $S$. De fato, como $\operatorname{cl}_{S}(M)=\operatorname{cl}_{X}(M) \cap S$, temos que

$$
S \backslash \mathrm{cl}_{S}(M)=S \backslash\left[\mathrm{cl}_{X}(M) \cap S\right]=\left[S \backslash \mathrm{cl}_{X}(M)\right] \cup(S \backslash S)=S \backslash \mathrm{cl}_{X}(M) .
$$

Logo, se $M$ não fosse denso em $S$, teríamos que $S \backslash \operatorname{cl}_{X}(M) \neq \emptyset$.

Afirmamos que $S \backslash \mathrm{cl}_{X}(M)$ é denso em si mesmo. De fato, se $p$ fosse um ponto isolado de $S \backslash \operatorname{cl}_{X}(M)$, então

$$
\{p\}=W \cap\left[S \backslash \mathrm{cl}_{X}(M)\right]=W \cap\left[X \backslash \mathrm{cl}_{X}(M)\right] \cap S
$$

para algum subconjunto aberto $W$ de $X$. Portanto, $p$ é um ponto isolado de $S$, já que $W \cap\left[X \backslash \mathrm{cl}_{X}(M)\right]$ é aberto em $X$. Contudo, isto é um absurdo, pois $S$ é denso em si mesmo.

Como $S \backslash \mathrm{cl}_{X}(M)$ é denso em si mesmo temos, por hipótese, que $S \backslash \mathrm{cl}_{X}(M)$ é abundante em $X$. Como $X$ é $\mathcal{D}$-forçado, da proposição 6.1.6 segue que $S \backslash \operatorname{cl}_{X}(M)$ contém um $\mathcal{D}$ pedaço $P=U \cap D$, onde $U$ é um aberto não vazio de $X$ e $D \in \mathcal{D}$. Logo, $U \cap D \subset S$ e $(U \cap D) \cap \operatorname{cl}_{X}(M)=\emptyset$.

Se existisse $V \in \mathcal{V}$ tal que $U \cap V \neq \emptyset$, então $U \cap V \cap D_{V} \neq \emptyset$, pois $D_{V}$ é denso em $X$. Como $V \cap D_{V} \subset M \subset S$, teríamos que $U \cap V \cap D_{V} \subset M \subset S$. Logo, $U \cap V \subset \operatorname{cl}_{X}(U \cap V)=\operatorname{cl}_{X}\left(U \cap V \cap D_{V}\right) \subset \operatorname{cl}_{X}(M)$. Portanto, $\emptyset \neq(U \cap V) \cap D \subset \operatorname{cl}_{X}(M)$, o que é um absurdo, pois

$$
\emptyset \neq(U \cap V) \cap D \cap \operatorname{cl}_{X}(M) \subset(U \cap D) \cap \operatorname{cl}_{X}(M)=\emptyset .
$$

Logo, $U \cap V=\emptyset$, para todo $V \in \mathcal{V}$, o que contraria a maximalidade de $\mathcal{V}$. Portanto, $M$ é denso em $S$. Além disso, se todo $D \in \mathcal{D}$ for $\mu$-resolúvel, $M$ também o será, devido ao lema 6.1.12. Como $M$ é denso em $S, S$ também será $\mu$-resolúvel, pela proposição 2.2.4. Logo, $X$ é hereditariamente $\mu$-resolúvel. 
Encerraremos esta seção observando que se $X$ é um espaço topológico NODEC, então todo subespaço denso em si mesmo de $X$ é abundante.

Com efeito, seja $A$ um subespaço denso em si mesmo de $X$. Suponhamos, por absurdo, que $A$ seja raro. Seja $p \in A$. Temos que $A \backslash\{p\} \subset A$. Logo, $\overline{A \backslash\{p\}} \subset \bar{A}$ e, portanto, $\operatorname{int}(\overline{A \backslash\{p\}}) \subset \operatorname{int}(\bar{A})=\emptyset$. Daí, segue que $A \backslash\{p\}$ é raro e, portanto, é fechado em $X$, uma vez que $X$ é NODEC. Como

$$
X \backslash(A \backslash\{p\})=(X \backslash A) \cup\{p\}
$$

é aberto em $X$, temos que

$$
A \cap[(X \backslash A) \cup\{p\}]=\{p\}
$$

é aberto em $A$, o que é absurdo, pois $A$ não tem pontos isolados.

\subsection{Famílias independentes de partições}

Dada uma seqüência de cardinais $\vec{\lambda}=\left\langle\lambda_{\zeta}: \zeta<\mu\right\rangle$, consideremos $\mathbb{F} \mathbb{I N}(\vec{\lambda}):=\left\{\epsilon: \epsilon\right.$ é uma função tal que dom $\epsilon \in[\mu]^{<\omega}$ e $\epsilon(\zeta) \in \lambda_{\zeta}$, para todo $\left.\zeta \in \operatorname{dom} \epsilon\right\}$.

Seja $\lambda$ um cardinal. Notemos que se $\lambda_{\zeta}=\lambda$, para todo $\zeta<\mu$, então $\mathbb{F} \mathbb{N}(\vec{\lambda})=\operatorname{Fn}(\mu, \lambda)$.

Consideremos $S$ um conjunto, $\vec{\lambda}=\left\langle\lambda_{\zeta}: \zeta<\mu\right\rangle$ uma seqüência de cardinais e $\mathbb{B}=\left\{\left\langle B_{\zeta}^{i}: i<\lambda_{\zeta}\right\rangle: \zeta<\mu\right\}$ uma família de partições de $S$.

Definição 6.2.1. Seja $\kappa$ um cardinal. Dizemos que $\mathbb{B}$ é $\kappa$-independente se, para cada $\epsilon \in \mathbb{F} \mathbb{N}(\vec{\lambda})$, a cardinalidade do conjunto

$$
\mathbb{B}[\epsilon]:=\bigcap\left\{B_{\zeta}^{\epsilon(\zeta)}: \zeta \in \operatorname{dom} \epsilon\right\}
$$


é maior ou igual a $\kappa$. Diremos que $\mathbb{B}$ é independente se, e somente se, $\mathbb{B}$ for 1 -independente (isto é, $\mathbb{B}[\epsilon] \neq \emptyset$, para toda $\epsilon \in \mathbb{F} \mathbb{N}(\vec{\lambda})$ ).

Definição 6.2.2. Dizemos que a família $\mathbb{B}$ é separante se, para cada $\{\alpha, \beta\} \in[S]^{2}$, existem $\zeta<\mu$ e $\{\rho, \nu\} \in\left[\lambda_{\zeta}\right]^{2}$ tais que $\alpha \in B_{\zeta}^{\rho}$ e $\beta \in B_{\zeta}^{\nu}$.

Denotaremos por $\tau_{\mathbb{B}}$ a topologia sobre $S$ gerada pela subbase

$$
\left\{B_{\zeta}^{i}: \zeta<\mu, i<\lambda_{\zeta}\right\}
$$

e designaremos o espaço topológico $\left(S, \tau_{\mathbb{B}}\right)$ por $X_{\mathbb{B}}$.

Proposição 6.2.3. O espaço topológico $X_{\mathbb{B}}$ é zero-dimensional e a família $\{\mathbb{B}[\epsilon]: \epsilon \in$ $\mathbb{F} \mathbb{N}(\vec{\lambda})\}$ constitui uma base de abertos para o espaço em questão. Além disso, $X_{\mathbb{B}}$ é $T_{2}$ se, e somente se, $\mathbb{B}$ é separante.

Demonstração. Observamos, primeiramente, que a família

$$
\{\mathbb{B}[\epsilon]: \epsilon \in \mathbb{F} \mathbb{N}(\vec{\lambda})\}
$$

constitui uma base de abertos para $X_{\mathbb{B}}$ pois esta é igual, a menos do conjunto vazio, à coleção de todas as intersecções finitas de elementos da subbase

$$
\left\{B_{\zeta}^{i}: \zeta<\mu, i<\lambda_{\zeta}\right\}
$$

que gera a topologia de $X_{\mathbb{B}}$.

Dada $\epsilon \in \mathbb{F} \mathbb{N}(\vec{\lambda})$, temos que $\mathbb{B}[\epsilon]$ é aberto em $X_{\mathbb{B}}$. Mostremos que $\mathbb{B}[\epsilon]$ é, também, fechado em $X_{\mathbb{B}}$. Com efeito,

$$
\mathbb{B}[\epsilon]=\bigcap_{\zeta \in \operatorname{dom} \epsilon} B_{\zeta}^{\epsilon(\zeta)}
$$


Assim,

$$
S \backslash \mathbb{B}[\epsilon]=S \backslash\left(\bigcap_{\zeta \in \operatorname{dom} \epsilon} B_{\zeta}^{\epsilon(\zeta)}\right)=\bigcup_{\zeta \in \operatorname{dom} \epsilon}\left(S \backslash B_{\zeta}^{\epsilon(\zeta)}\right)
$$

Logo,

$$
S \backslash \mathbb{B}[\epsilon]=\bigcup_{\zeta \in \operatorname{dom} \epsilon, i<\lambda_{\zeta}, i \neq \epsilon(\zeta)} B_{\zeta}^{i}
$$

pois $\left\langle B_{\zeta}^{i}: i<\lambda_{\zeta}\right\rangle$ é uma partição de $S$, para todo $\zeta \in \operatorname{dom} \epsilon$. Portanto, $S \backslash \mathbb{B}[\epsilon]$ é aberto em $X_{\mathbb{B}}$, o que implica que $\mathbb{B}[\epsilon]$ é fechado em $X_{\mathbb{B}}$. Logo, $X_{\mathbb{B}}$ é zero-dimensional.

Mostremos, por fim, que $X_{\mathbb{B}}$ é $T_{2}$ se, e somente se, $\mathbb{B}$ é separante. Se $\mathbb{B}$ for separante, dado $\{\alpha, \beta\} \in[S]^{2}$, existem $\zeta<\mu$ e $\{\rho, \nu\} \in\left[\lambda_{\zeta}\right]^{2}$ tais que $\alpha \in B_{\zeta}^{\rho}$ e $\beta \in B_{\zeta}^{\nu}$. Como $\left\langle B_{\zeta}^{i}: i<\lambda_{\zeta}\right\rangle$ é uma partição de $S$, segue que $B_{\zeta}^{\rho} \cap B_{\zeta}^{\nu}=\emptyset$. Como $B_{\zeta}^{\rho}$ e $B_{\zeta}^{\nu}$ são abertos em $X_{\mathbb{B}}$, concluímos que $X_{\mathbb{B}}$ é $T_{2}$. Reciprocamente, se $X_{\mathbb{B}}$ é $T_{2}$, dados $\{\alpha, \beta\} \in[S]^{2}$, existem $\delta, \epsilon \in \mathbb{F} \mathbb{N}(\vec{\lambda})$ tais que $\alpha \in \mathbb{B}[\delta], \beta \in \mathbb{B}[\epsilon]$ e $\mathbb{B}[\delta] \cap \mathbb{B}[\epsilon]=\emptyset$. Em particular, $\alpha \notin \mathbb{B}[\epsilon]=\cap_{\zeta \in \operatorname{dom} \epsilon} B_{\zeta}^{\epsilon(\zeta)}$. Logo, existe $\zeta \in \operatorname{dom} \epsilon$ tal que $\alpha \notin B_{\zeta}^{\epsilon(\zeta)}$. Claramente, $\beta \in B_{\zeta}^{\epsilon(\zeta)}$.

Se $\zeta \in \operatorname{dom} \delta$, então $\delta(\zeta) \neq \epsilon(\zeta)$, pois $\alpha \in \mathbb{B}[\delta]=\cap_{\zeta \in \operatorname{dom} \delta} B_{\zeta}^{\delta(\zeta)}$ e, portanto, $\alpha \in B_{\zeta}^{\delta(\zeta)}$. Neste caso, $\mathbb{B}$ é separante.

Se $\zeta \notin \operatorname{dom} \delta$, consideremos $\delta^{*} \in \mathbb{F} \mathbb{N}(\vec{\lambda})$ tal que $\operatorname{dom} \delta^{*}=\operatorname{dom} \delta \cup\{\zeta\}, \delta^{*} \uparrow_{\operatorname{dom} \delta}=\delta$ e $\delta^{*}(\zeta)=\rho$, onde $\rho<\lambda_{\zeta}$ é tal que $\alpha \in B_{\zeta}^{\rho}$. Este $\rho$ existe e é único, pois $\left\langle B_{\zeta}^{i}: i<\lambda_{\zeta}\right\rangle$ é uma partição de $X_{\mathbb{B}}$. Evidentemente, $\mathbb{B}\left[\delta^{*}\right] \cap \mathbb{B}[\epsilon]=\emptyset$, pois $\mathbb{B}\left[\delta^{*}\right] \subset \mathbb{B}[\delta]$. Além disso, $\alpha \in \mathbb{B}\left[\delta^{*}\right]$. Temos que $\delta^{*}(\zeta) \neq \epsilon(\zeta)$, pois $\alpha \in B_{\zeta}^{\delta^{*}(\zeta)}$ e $\alpha \notin B_{\zeta}^{\epsilon(\zeta)}$. Logo, $\mathbb{B}$ é separante.

Teorema 6.2.4. Sejam $\kappa, \lambda$ e $\mu$ cardinais infinitos e seja

$$
\mathbb{B}=\left\{\left\langle B_{\alpha}^{0}, B_{\alpha}^{1}\right\rangle: \alpha<\lambda\right\}
$$

uma familia separante e $\mu$-independente de partiçôes de $\kappa$. Há um homeomorfismo entre o espaço topológico $X_{\mathbb{B}}=\left(\kappa, \tau_{\mathbb{B}}\right)$ e um subespaço $\mu$-denso de $2^{\lambda}$. 
Demonstração. Seja

$$
\psi: X_{\mathbb{B}} \rightarrow 2^{\lambda}
$$

dada por

$$
\psi(x)(\alpha)=\left\{\begin{array}{lll}
0 & \text { se } & x \in B_{\alpha}^{0} \\
1 & \text { se } & x \in B_{\alpha}^{1}
\end{array}\right.
$$

para cada $\alpha<\lambda$.

Tal função está bem definida pois, para todo $\alpha<\lambda,\left\langle B_{\alpha}^{0}, B_{\alpha}^{1}\right\rangle$ é uma partição de $\kappa$.

Mostremos, primeiramente, que $\psi$ é injetora. Para tanto, consideremos $x, y \in \kappa$ tais que $\psi(x)=\psi(y)$. Então, $\psi(x)(\zeta)=\psi(y)(\zeta)$, para todo $\zeta<\lambda$. Logo, $x \in B_{\zeta}^{\psi(x)(\zeta)}=B_{\zeta}^{\psi(y)(\zeta)}$ e $y \in B_{\zeta}^{\psi(x)(\zeta)}=B_{\zeta}^{\psi(y)(\zeta)}$, para todo $\zeta<\lambda$. Como, por hipótese, $\mathbb{B}$ é separante, segue que $x=y$. Portanto, $\psi$ é injetora.

Provaremos, agora, que $\psi\left[X_{\mathbb{B}}\right]$ é $\mu$-denso em $2^{\lambda}$. Um aberto básico de $2^{\lambda}$ é da forma

$$
\Omega(p)=\prod_{\zeta<\lambda} A_{\zeta}
$$

onde $p \in \operatorname{Fn}(\lambda, 2) \mathrm{e}$

$$
A_{\zeta}:= \begin{cases}\{p(\zeta)\} & \text { se } \quad \zeta \in \operatorname{dom} p \\ 2 & \text { se } \zeta \notin \operatorname{dom} p\end{cases}
$$

Seja $p \in \operatorname{Fn}(\lambda, 2)$, qualquer. Como $\mathbb{B}$ é $\mu$-independente, temos que

$$
\left|\bigcap\left\{B_{\zeta}^{p(\zeta)}: \zeta \in \operatorname{dom} p\right\}\right| \geq \mu
$$

Para todo

$$
x \in \bigcap_{\zeta \in \operatorname{dom} p} B_{\zeta}^{p(\zeta)}
$$


temos que $\psi(x)(\zeta)=p(\zeta)$, qualquer que seja $\zeta \in \operatorname{dom} p$. Assim, $\psi(x) \in \Omega(p)$. Como $\psi$ é injetora, temos que $\left|\psi\left[X_{\mathbb{B}}\right] \cap \Omega(p)\right| \geq \mu$. Logo, $\psi\left[X_{\mathbb{B}}\right]$ é $\mu$-denso em $2^{\lambda}$.

Mostremos, por fim, que $\psi$ é um homeomorfismo sobre sua imagem. De fato, $\psi$ é contínua pois, se $\Omega(p)$ é um aberto básico de $2^{\lambda}$, então

$$
\psi^{-1}[\Omega(p)]=\bigcap_{\zeta \in \operatorname{dom} p} B_{\zeta}^{p(\zeta)}
$$

é aberto em $X_{\mathbb{B}}$. Além disso, $\psi$ é aberta. Com efeito, da proposição 6.2 .3 vem que

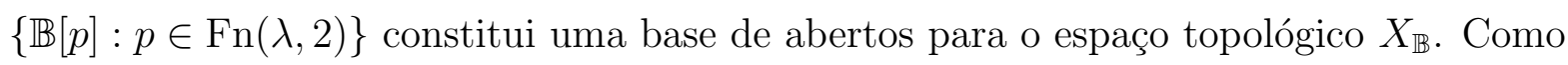

$$
\psi[\mathbb{B}[p]]=\Omega(p) \cap \psi\left[X_{\mathbb{B}}\right]
$$

segue que $\psi[\mathbb{B}[p]]$ é aberto em $\psi\left[X_{\mathbb{B}}\right]$.

Teorema 6.2.5. Para cada cardinal infinito $\kappa$, existe uma família

$$
\mathbb{B}=\left\{\left\langle B_{\zeta}^{i}: i<\kappa\right\rangle: \zeta<2^{\kappa}\right\}
$$

de partições de $\kappa$ que é separante e $\kappa$-independente.

Demonstração. Do teorema de Hewitt-Marczewski-Pondiczery, segue que $\kappa^{2^{\kappa}}$ contém um subconjunto $Z$ de cardinalidade $\kappa$, que é $\kappa$-denso em $\kappa^{2^{\kappa}}$. Vamos, a partir de $Z$, construir uma família

$$
\mathbb{B}=\left\{\left\langle B_{\zeta}^{i}: i<\kappa\right\rangle: \zeta<2^{\kappa}\right\}
$$

de partições de $\kappa$, que é separante e $\kappa$-independente.

Como $|Z|=\kappa$, existe uma bijeção $\psi: \kappa \rightarrow Z$. Fixemos $\zeta<2^{\kappa}$. Para cada $i<\kappa$, definamos

$$
B_{\zeta}^{i}=\{x \in \kappa: \psi(x)(\zeta)=i\}
$$


É fácil ver que $\left\langle B_{\zeta}^{i}: i<\kappa\right\rangle$ é uma partição de $\kappa$, para todo $\zeta<2^{\kappa}$ e, portanto, que

$$
\mathbb{B}=\left\{\left\langle B_{\zeta}^{i}: i<\kappa\right\rangle: \zeta<2^{\kappa}\right\}
$$

é uma família de partições de $\kappa$.

A fim de provar que $\mathbb{B}$ é $\kappa$-independente, consideremos $p$ um elemento arbitrário de $\operatorname{Fn}\left(2^{\kappa}, \kappa\right)$ e mostremos que $\left|\bigcap_{\zeta \in \operatorname{dom} p} B_{\zeta}^{p(\zeta)}\right| \geq \kappa$.

Seja $\Omega(p)$ o aberto básico de $\kappa^{2^{\kappa}}$ dado por

$$
\Omega(p)=\prod_{\zeta<2^{\kappa}} A_{\zeta}
$$

onde

$$
A_{\zeta}:= \begin{cases}\{p(\zeta)\} & \text { se } \quad \zeta \in \operatorname{dom} p \\ \kappa & \text { se } \zeta \notin \operatorname{dom} p\end{cases}
$$

Como $Z$ é $\kappa$-denso em $\kappa^{2^{\kappa}}$, temos que $|Z \cap \Omega(p)| \geq \kappa$.

Seja $y \in Z \cap \Omega(p)$. Consideremos $x \in \kappa$ tal que $y=\psi(x)$. Temos que $x \in \cap_{\zeta \in \operatorname{dom} p} B_{\zeta}^{p(\zeta)}$, pois $\psi(x)(\zeta)=y(\zeta)=p(\zeta)$, para todo $\zeta \in \operatorname{dom} p$. Uma vez que $\psi$ é bijetora, segue que

$$
\left|\bigcap_{\zeta \in \operatorname{dom} p} B_{\zeta}^{p(\zeta)}\right| \geq \kappa
$$

Portanto, $\mathbb{B}$ é $\kappa$-independente.

Mostremos, finalmente, que $\mathbb{B}$ é separante. Seja $\{\alpha, \beta\} \in[\kappa]^{2}$. Como $\psi$ é injetora, temos que $\psi(\alpha) \neq \psi(\beta)$ e, portanto, existe $\zeta \in 2^{\kappa}$ tal que $\psi(\alpha)(\zeta) \neq \psi(\beta)(\zeta)$. Logo, $\alpha \in B_{\zeta}^{\psi(\alpha)(\zeta)}$ e $\beta \in B_{\zeta}^{\psi(\beta)(\zeta)}$. Portanto, $\mathbb{B}$ é separante. 
Apresentaremos, por fim, o principal teorema desta seção.

Teorema 6.2.6. Sejam $\kappa$ um cardinal infinito e $\mathbb{B}=\left\{\left\langle B_{\xi}^{0}, B_{\xi}^{1}\right\rangle: \xi<2^{\kappa}\right\}$ uma família $\kappa$-independente de partições de $\kappa$. Seja $\mathcal{D}$ uma família não vazia de subconjuntos $\kappa$-densos do espaço topológico $X_{\mathbb{B}}$. Existe uma família $\kappa$-independente e separante $\mathbb{C}=\left\{\left\langle C_{\xi}^{0}, C_{\xi}^{1}\right\rangle\right.$ : $\left.\xi<2^{\kappa}\right\}$ de partições de $\kappa$, satisfazendo as seguintes propriedades:

(1) $S e D \in \mathcal{D}$, então $D$ é $\kappa$-denso em $X_{\mathbb{C}}\left(e\right.$, portanto, $\left.\Delta\left(X_{\mathbb{C}}\right)=\kappa\right)$;

(2) $X_{\mathbb{C}}$ é $\mathcal{D}$-forçado;

(3) Se $N \in[\kappa]^{<\kappa}$, então $N$ é um subconjunto raro de $X_{\mathbb{C}}$;

(4) $X_{\mathbb{C}}$ é $N O D E C$.

Além disso, se $J \subset 2^{\kappa}$ é tal que $\left|2^{\kappa} \backslash J\right|=2^{\kappa}$, podemos assumir que

(5) $\mathbb{C} \uparrow J=\mathbb{B} \uparrow J$.

Demonstração. Seja $J \subset 2^{\kappa}$ tal que $\left|2^{\kappa} \backslash J\right|=2^{\kappa}$. Definamos $I=2^{\kappa} \backslash J$. Consideremos $I=I_{0} \cup I^{\prime}$ uma partição de $I$, tal que $\left|I_{0}\right|=\kappa^{<\kappa}$ e $\left|I^{\prime}\right|=2^{\kappa}$. Observamos que é possível obter uma tal partição, pois $\kappa^{<\kappa} \leq 2^{\kappa}$.

Particionemos $I_{0}$ em uma coleção de subconjuntos enumeráveis e não vazios $J_{A, \alpha}$, para todo $A \in[\kappa]^{<\kappa}$ e todo $\alpha \in \kappa \backslash A$. Observamos, novamente, que é possível obter uma tal partição, uma vez que $\left|[\kappa]^{<\kappa}\right|=\kappa^{<\kappa}$ e $|\kappa \backslash A|=\kappa$.

Seja $\xi \in I_{0}$. Existem únicos $A \in[\kappa]^{<\kappa}$ e $\alpha \in \kappa \backslash A$ tais que $\xi \in J_{A, \alpha}$. Definamos

$$
C_{\xi}^{0}=\left(B_{\xi}^{0} \cup A\right) \backslash\{\alpha\}
$$

e

$$
C_{\xi}^{1}=\left(B_{\xi}^{1} \backslash A\right) \cup\{\alpha\}
$$


Temos que $C_{\xi}^{0} \cap C_{\xi}^{1}=\emptyset$ e $C_{\xi}^{0} \cup C_{\xi}^{1}=\kappa$, para todo $\xi \in I_{0}$. Portanto, $\left\langle C_{\xi}^{0}, C_{\xi}^{1}\right\rangle$ constitui uma partição de $\kappa$, para todo $\xi \in I_{0}$.

Fixemos uma enumeração $\left\{F_{\nu}: \nu<2^{\kappa}\right\}$ de $[\kappa]^{\kappa}$. Por indução transfinita em $\nu<2^{\kappa}$, definiremos

- Funções parciais finitas $\eta_{\nu} \in \operatorname{Fn}\left(2^{\kappa}, 2\right)$ e conjuntos

$$
K_{\nu} \subset I^{\prime} \backslash\left[\bigcup_{\zeta<\nu} K_{\zeta} \cup \bigcup\left\{\operatorname{dom} \eta_{\zeta}: \zeta<\nu\right\}\right]
$$

de modo que $K_{\nu}=\emptyset$ ou $\left|K_{\nu}\right|=\kappa$;

- partições $\left\langle C_{\sigma}^{0}, C_{\sigma}^{1}\right\rangle$ de $\kappa$, para todo $\sigma \in K_{\nu}$, tais que

$$
\left|D \cap \mathbb{B}_{\nu}[\epsilon]\right|=\kappa
$$

quaisquer que sejam $\epsilon \in \operatorname{Fn}\left(2^{\kappa}, 2\right)$ e $D \in \mathcal{D}$, onde

$$
\mathbb{B}_{\nu}=\left\{\left\langle C_{\sigma}^{0}, C_{\sigma}^{1}\right\rangle: \sigma \in I_{\nu}\right\} \cup\left\{\left\langle B_{\sigma}^{0}, B_{\sigma}^{1}\right\rangle: \sigma \in 2^{\kappa} \backslash I_{\nu}\right\}
$$

com

$$
I_{\nu}=I_{0} \cup \bigcup_{\zeta<\nu} K_{\zeta}
$$

Salientamos que, desta forma, todo subconjunto $D$ de $\mathcal{D}$ será $\kappa$-denso em $X_{\mathbb{B}_{\nu}}$.

$$
\nu=0
$$

Observemos, primeiramente, que

$$
\mathbb{B}_{0}=\left\{\left\langle C_{\sigma}^{0}, C_{\sigma}^{1}\right\rangle: \sigma \in I_{0}\right\} \cup\left\{\left\langle B_{\sigma}^{0}, B_{\sigma}^{1}\right\rangle: \sigma \in 2^{\kappa} \backslash I_{0}\right\}
$$


Como, por hipótese, $|\mathbb{B}[\epsilon] \cap D|=\kappa$, para toda $\epsilon \in \operatorname{Fn}\left(2^{\kappa}, 2\right)$ e todo $D \in \mathcal{D}$, temos que $\left|\mathbb{B}_{0}[\epsilon] \cap D\right|=\kappa$, quaisquer que sejam $\epsilon \in \operatorname{Fn}\left(2^{\kappa}, 2\right)$ e $D \in \mathcal{D}$.

$\nu$ ordinal limite

Sejam $\epsilon \in \operatorname{Fn}\left(2^{\kappa}, 2\right)$ e $D \in \mathcal{D}$, quaisquer.

Se dom $\epsilon \subset\left(2^{\kappa} \backslash I_{\nu}\right) \cup I_{0}$, então $\mathbb{B}_{\nu}[\epsilon]=\mathbb{B}_{0}[\epsilon]$. Logo, $\left|\mathbb{B}_{\nu}[\epsilon] \cap D\right|=\kappa$.

Se $\operatorname{dom} \epsilon \cap\left(\cup_{\zeta<\nu} K_{\zeta}\right) \neq \emptyset$, tomamos $\lambda=\max \left\{\zeta<\nu: \operatorname{dom} \epsilon \cap K_{\zeta} \neq \emptyset\right\}$. Como $\nu$ é ordinal limite, temos que $\lambda+1<\nu$. Além disso, $\mathbb{B}_{\nu}[\epsilon]=\mathbb{B}_{\lambda+1}[\epsilon]$. Portanto, das hipóteses de indução, segue que $\left|\mathbb{B}_{\nu}[\epsilon] \cap D\right|=\kappa$.

$$
\nu+1
$$

Consideremos, agora, os passos sucessores. Assumiremos que as hipóteses da indução transfinita estão satisfeitas para $\nu$ e distinguiremos dois casos.

Caso 1. $F_{\nu}$ contém um $\left(\mathcal{D}, X_{\mathbb{B}_{\nu}}\right)$-pedaço, isto é, $F_{\nu} \supset D \cap \mathbb{B}_{\nu}\left[\eta_{\nu}\right]$, para alguma $\eta_{\nu} \in F n\left(2^{\kappa}, 2\right)$ e algum $D \in \mathcal{D}$.

Escolhamos $\epsilon \in \operatorname{Fn}\left(2^{\kappa}, 2\right)$ tal que $D \cap \mathbb{B}_{\nu}[\epsilon] \subset F_{\nu}$, para algum $D \in \mathcal{D}$ e façamos $\eta_{\nu}=\epsilon$. Tomemos $K_{\nu}=\emptyset$.

Teremos, neste caso, que $I_{\nu}=I_{\nu+1}$ e, portanto, $\mathbb{B}_{\nu}=\mathbb{B}_{\nu+1}$.

Logo, nossas hipóteses de indução são trivialmente verificadas.

Caso 2. $F_{\nu}$ não contém um $\left(\mathcal{D}, X_{\mathbb{B}_{\nu}}\right)$-pedaço, isto é, $\left(D \cap \mathbb{B}_{\nu}[\epsilon]\right) \backslash F_{\nu} \neq \emptyset$, para toda $\epsilon \in F n\left(2^{\kappa}, 2\right)$ e todo $D \in \mathcal{D}$. 
Neste caso, escolheremos

$$
K_{\nu} \subset I^{\prime} \backslash\left[\bigcup_{\zeta<\nu} K_{\zeta} \cup \bigcup\left\{\operatorname{dom} \eta_{\zeta}: \zeta<\nu\right\}\right]
$$

um conjunto de cardinalidade $\kappa$. Seja $K_{\nu}=\left\{\gamma_{\nu, i}: i<\kappa\right\}$ uma enumeração de $K_{\nu}$. Façamos, ainda, $\eta_{\nu}=\emptyset$.

Queremos modificar as partições $\left\langle B_{\sigma}^{0}, B_{\sigma}^{1}\right\rangle$ de $\kappa$, para $\sigma \in K_{\nu}$, de maneira a tornar o conjunto $F_{\nu}$ fechado e discreto em $X_{\mathbb{B}_{\nu+1}}$.

Definamos, portanto, para todo $i<\kappa$,

$$
C_{\gamma_{\nu, i}}^{0}=\left(B_{\gamma_{\nu, i}}^{0} \backslash F_{\nu}\right) \cup\{i\}
$$

e

$$
C_{\gamma_{\nu, i}}^{1}=\left(B_{\gamma_{\nu, i}}^{1} \cup F_{\nu}\right) \backslash\{i\} .
$$

Para cada $i<\kappa$, temos que

$$
F_{\nu} \cap C_{\gamma_{\nu, i}}^{0} \subset\{i\} .
$$

Portanto, $F_{\nu}$ é fechado e discreto em $X_{\mathbb{B}_{\nu+1}}$.

Finalmente, suponhamos por absurdo que

$$
\left|D \cap \mathbb{B}_{\nu+1}[\epsilon]\right|<\kappa
$$

para algum $D \in \mathcal{D}$ e alguma $\epsilon \in \operatorname{Fn}\left(2^{\kappa}, 2\right)$.

Como

$$
\left|\left(D \cap \mathbb{B}_{\nu+1}[\epsilon]\right) \cup\left\{i \in \kappa: \gamma_{\nu, i} \in \operatorname{dom} \epsilon \cap K_{\nu}\right\}\right|<\kappa
$$

existe $\xi \in I_{0} \backslash$ dom $\epsilon$ tal que

$$
\left(D \cap \mathbb{B}_{\nu+1}[\epsilon]\right) \cup\left\{i \in \kappa: \gamma_{\nu, i} \in \operatorname{dom} \epsilon \cap K_{\nu}\right\} \subset C_{\xi}^{0} .
$$

Seja $\epsilon^{*} \in \operatorname{Fn}\left(2^{\kappa}, 2\right)$ tal que $\operatorname{dom} \epsilon^{*}=\operatorname{dom} \epsilon \cup\{\xi\},\left.\epsilon^{*}\right|_{\operatorname{dom} \epsilon}=\epsilon$ e $\epsilon^{*}(\xi)=1$. 
Temos que

$$
D \cap \mathbb{B}_{\nu+1}\left[\epsilon^{*}\right]=\emptyset
$$

Como $\mathbb{B}_{\nu}\left[\epsilon^{*}\right]=\mathbb{B}_{\nu}[\epsilon] \cap C_{\xi}^{1}$, temos que se $x \in \mathbb{B}_{\nu}\left[\epsilon^{*}\right]$, então $x \neq i$, para todo $i<\kappa$ tal que $\gamma_{\nu, i} \in \operatorname{dom} \epsilon \cap K_{\nu}$, uma vez que $C_{\xi}^{0} \cap C_{\xi}^{1}=\emptyset$. Portanto,

$$
\mathbb{B}_{\nu+1}\left[\epsilon^{*}\right] \supset \mathbb{B}_{\nu}\left[\epsilon^{*}\right] \backslash F_{\nu}
$$

Conseqüentemente,

$$
\emptyset=D \cap \mathbb{B}_{\nu+1}\left[\epsilon^{*}\right] \supset\left(D \cap \mathbb{B}_{\nu}\left[\epsilon^{*}\right]\right) \backslash F_{\nu} \neq \emptyset
$$

uma contradição.

Portanto, o processo de indução tranfinita está completo.

Definamos

$$
I_{2^{\kappa}}=I_{0} \cup \bigcup_{\zeta<2^{\kappa}} K_{\zeta}
$$

e

$$
\mathbb{C}=\left\{\left\langle C_{\sigma}^{0}, C_{\sigma}^{1}\right\rangle: \sigma \in I_{2^{\kappa}}\right\} \cup\left\{\left\langle B_{\sigma}^{0}, B_{\sigma}^{1}\right\rangle: \sigma \in 2^{\kappa} \backslash I_{2^{\kappa}}\right\} .
$$

Mostremos que a família de partições de $\kappa$ acima explicitada possui as características desejadas. Em primeiro lugar, observamos que $\mathbb{C}$ é separante. De fato, se $\alpha, \beta \in \kappa$ e $\alpha \neq \beta$, tomando $\xi \in J_{\{\alpha\}, \beta} \subset I_{0}$, decorre que $\alpha \in C_{\xi}^{0}$ e $\beta \in C_{\xi}^{1}$.

Das hipóteses da indução transfinita concluímos que $\mathbb{C}$ é $\kappa$-independente e que (1) vale.

Se $A \in[\kappa]^{<\kappa}$ e $\alpha \in \kappa \backslash A$, então $A \subset C_{\xi}^{0}$ e $\alpha \in C_{\xi}^{1}$, para todo $\xi \in J_{A, \alpha}$. Logo $\alpha \notin \operatorname{cl}_{X_{\mathbb{C}}}(A)$. Portanto, $A$ é fechado em $X_{\mathbb{C}}$. Como $\Delta\left(X_{\mathbb{C}}\right)=\kappa$, concluímos que $A$ é raro em $X_{\mathbb{C}}$. Assim, (3) está satisfeita.

Seja $F$ um subconjunto raro de $X_{\mathbb{C}}$. Queremos mostrar que $F$ é fechado em $X_{\mathbb{C}}$. De acordo com o parágrafo anterior, podemos supor que $|F|=\kappa$. Logo, existe $\nu<2^{\kappa}$ tal que 
$F=F_{\nu}$. Se neste passo da indução transfinita estivéssemos no caso 1 , existiria $D \in \mathcal{D}$ tal que $F \supset D \cap \mathbb{B}_{\nu}\left[\eta_{\nu}\right]$. Como $\mathbb{B}_{\nu}\left[\eta_{\nu}\right]=\mathbb{C}\left[\eta_{\nu}\right]$, teríamos que $F \supset D \cap \mathbb{C}\left[\eta_{\nu}\right]$ e, portanto

$$
\mathrm{cl}_{X_{\mathbb{C}}}(F) \supset \mathrm{cl}_{X_{\mathbb{C}}}\left(D \cap \mathbb{C}\left[\eta_{\nu}\right]\right)=\mathrm{cl}_{X_{\mathbb{C}}}\left(\mathbb{C}\left[\eta_{\nu}\right]\right) \supset \mathbb{C}\left[\eta_{\nu}\right]
$$

o que é absurdo, pois $F$ é raro em $X_{\mathbb{C}}$. Esta contradição mostra que no $\nu$-ésimo passo da indução transfinita estamos no caso 2. Logo, $F=F_{\nu}$ é fechado e discreto em $X_{\mathbb{B}_{\nu+1}}$ e, portanto, em $X_{\mathbb{C}}$. Conseqüentemente, $X_{\mathbb{C}}$ é NODEC, ou seja, vale (4).

Resta verificar que $X_{\mathbb{C}}$ é $\mathcal{D}$-forçado, isto é, que (2) é satisfeita. De acordo com a proposição 6.1.6, basta mostrar que todo subconjunto abundante de $X_{\mathbb{C}}$ contém um $\left(\mathcal{D}, X_{\mathbb{C}}\right)$ pedaço. Seja $E$ um subconjunto abundante de $X_{\mathbb{C}}$. De (3), segue que $|E|=\kappa$ e, portanto, existe $\nu<2^{\kappa}$ tal que $F_{\nu}=E$. No $\nu$-ésimo passo da indução transfinita devemos estar no caso 1 pois $E$ é, por hipótese, abundante em $X_{\mathbb{C}}$. Logo, existem $\eta_{\nu} \in \operatorname{Fn}\left(2^{\kappa}, 2\right)$ e $D \in \mathcal{D}$ tais que $E=F_{\nu} \supset D \cap \mathbb{B}_{\nu}\left[\eta_{\nu}\right]$. Como $\mathbb{C}\left[\eta_{\nu}\right]=B_{\nu}\left[\eta_{\nu}\right]$, segue que $E$ contém o $\left(\mathcal{D}, X_{\mathbb{C}}\right)$-pedaço $D \cap \mathbb{C}\left[\eta_{\nu}\right]$

Finalmente, (5) é trivialmente válido por construção.

Ressaltamos que todos os espaços topológicos $X_{\mathbb{C}}$ obtidos através de uma aplicação do teorema 6.2.6 são zero-dimensionais e $T_{2}$ e, portanto, completamente regulares. Além disso, decorre de (1) que tais espaços são, também, densos em si mesmo.

\subsection{Aplicações à resolubilidade}

Teorema 6.3.1. Para todo cardinal infinito $\kappa$, o cubo de Cantor $2^{2^{\kappa}}$ contém um subespaço denso e submaximal $X$, com $|X|=\Delta(X)=\kappa$. 
Demonstração. Fixemos uma família $\kappa$-independente

$$
\mathbb{B}=\left\{\left\langle B_{\xi}^{0}, B_{\xi}^{1}\right\rangle: \xi<2^{\kappa}\right\}
$$

de partições de $\kappa$ e seja $\mathcal{D}=\{\kappa\}$.

Aplicando o teorema 6.2.6, obtemos $\mathbb{C}$ uma família separante e $\kappa$-independente de partições de $\kappa$ que satisfaz as propriedades (1) a (4) do mesmo. Portanto, o espaço topológico $X_{\mathbb{C}}$ é, em particular, $\mathcal{D}$-forçado e verifica

$$
\Delta\left(X_{\mathbb{C}}\right)=\kappa=\left|X_{\mathbb{C}}\right|
$$

Com o auxílio da proposição 6.1 .10 concluímos que $X_{\mathbb{C}}$ é OHI e, como o espaço topológico em questão também é NODEC, da proposição 5.1.3 segue que $X_{\mathbb{C}}$ é submaximal.

Do teorema 6.2.4 decorre que o cubo de Cantor $2^{2^{\kappa}}$ contém um subespaço denso e submaximal $X$, tal que $|X|=\Delta(X)=\kappa$.

Definição 6.3.2. Dizemos que um espaço topológico $X$ é $C C C$ se não existe uma família não enumerável de subconjuntos abertos não vazios e dois a dois disjuntos de $X$.

Teorema 6.3.3. Seja $\left\{X_{i}\right\}_{i \in I}$ uma familia de espaços topológicos tais que, para cada subconjunto finito $F \subset I$, o espaço topológico $\prod_{i \in F} X_{i}$ é CCC. Então, $\prod_{i \in I} X_{i}$ é CCC.

Demonstração. Suponhamos, por absurdo, que exista $\left\{U_{\alpha}\right\}_{\alpha<\omega_{1}}$ uma família de subconjuntos abertos não vazios de $\prod_{i \in I} X_{i}$, dois a dois disjuntos.

Podemos supor que $U_{\alpha}$ é um aberto básico de $\prod_{i \in I} X_{i}$, para todo $\alpha<\omega_{1}$. Desta maneira, temos que cada $U_{\alpha}$ depende somente de um conjunto finito de coordenadas $A_{\alpha} \subset I$.

Do lema do $\Delta$-sistema segue que existe um conjunto não enumerável $A$ contido em $\omega_{1}$ tal que $\left\{A_{\alpha}: \alpha \in A\right\}$ forma um $\Delta$-sistema de raiz $R$. Temos que $R \neq \emptyset$, pois se $A_{i} \cap A_{j}=\emptyset$, então $U_{i} \cap U_{j} \neq \emptyset$. 
Consideremos $\Pi\left(U_{\alpha}\right)$ a projeção de $U_{\alpha}$ no espaço topológico $\prod_{i \in R} X_{i}$. Temos que $\left\{\Pi\left(U_{\alpha}\right): \alpha \in A\right\}$ é uma coleção não enumerável de subconjuntos abertos não vazios de $\prod_{i \in R} X_{i}$, dois a dois disjuntos, o que é absurdo pois, por hipótese, $\prod_{i \in R} X_{i}$ é CCC.

Do teorema 6.3.3 concluímos que o cubo de Cantor $2^{\kappa}$ é CCC, para todo cardinal $\kappa$. Observamos, ainda, que todo espaço topológico obtido através de uma aplicação do teorema 6.2.6 é CCC, pois é homeomorfo a um subespaço denso em um cubo de Cantor.

Lema 6.3.4. Todo subespaço de um espaço topológico NODEC é, também, NODEC.

Demonstração. Sejam $X$ um espaço topológico NODEC e $Y$ um subespaço de $X$. Seja $N$ um subconjunto raro de $Y$. Desta forma, $\operatorname{cl}_{Y}(N)$ não contém nenhum subconjunto aberto não vazio de $Y$. Afirmamos que $\operatorname{cl}_{X}(N)$ não contém nenhum subconjunto aberto não vazio de $X$. Do contrário, existiria $U$ um aberto não vazio de $X$ tal que $U \subset \operatorname{cl}_{X}(N)$. Como $\operatorname{cl}_{Y}(N)=\operatorname{cl}_{X}(N) \cap Y$, teríamos que $\operatorname{cl}_{Y}(N) \supset U \cap Y$. Como $U \subset \operatorname{cl}_{X}(N)$, temos que $U \cap N \neq \emptyset$. Como $N \subset Y$, temos que $U \cap Y$ é um aberto não vazio de $Y$, o que contradiz o fato de $N$ ser um subconjunto raro de $Y$. Portanto, $N$ é um subconjunto raro de $X$. Como $X$ é NODEC, segue que $N$ é fechado em $X$ e, portanto, $N=N \cap Y$ é fechado em $Y$. Logo, $Y$ é NODEC.

O próximo resultado generaliza o teorema 4.2, que afirma ser possível construir, para todo número natural $n>1$, um espaço topológico $n$-resolúvel que não é $(n+1)$-resolúvel.

Teorema 6.3.5. Para quaisquer dois cardinais infinitos $\mu<\kappa$, existe um espaço topológico completamente regular que é união disjunta de $\mu$ subespaços submaximais densos (e, portanto, é $\mu$-resolúvel), mas que não é $\mu^{+}$-resolúvel. 
Demonstração. De acordo com o teorema 6.2.5, é possível encontrar uma partição

$$
\left\langle D_{i}: i<\mu\right\rangle
$$

de $\kappa$ e uma família de partições

$$
\mathbb{B}=\left\{\left\langle B_{\xi}^{0}, B_{\xi}^{1}\right\rangle: \xi<2^{\kappa}\right\}
$$

de $\kappa$ tais que, para cada $i<\mu$ e $\epsilon \in \operatorname{Fn}\left(2^{\kappa}, 2\right)$, tenhamos

$$
\left|D_{i} \cap \mathbb{B}[\epsilon]\right| \geq \kappa
$$

Podemos, então, aplicar o teorema 6.2 .6 à família de partições $\mathbb{B}$ e à coleção $\mathcal{D}=$ $\left\{D_{i}: i<\mu\right\}$ de subconjuntos $\kappa$-densos de $X$, a fim de obter $\mathbb{C}$ uma família separante e $\kappa$-independente de partições de $\kappa$ satisfazendo as propriedades (1) a (4) do teorema em questão. Afirmamos que o espaço topológico $X_{\mathbb{C}}$ possui as características desejadas.

Da propriedade (1) do teorema 6.2.6 segue que $D_{i}$ é denso em $X_{\mathbb{C}}$, para todo $i<\mu$. Como $X_{\mathbb{C}}$ é NODEC, do lema 6.3 .4 segue que $D_{i}$ também o é, para todo $i<\mu$. Como os elementos de $\mathcal{D}$ particionam $\kappa$, do lema 6.1.10 decorre que cada $D_{i}$ é OHI e, portanto, submaximal. Todavia, uma vez que $X_{\mathbb{C}}$ é CCC, $\mathcal{D}$-forçado e $|\mathcal{D}|=\mu \geq \omega$, da proposição 6.1.11 segue que $X_{\mathbb{C}}$ não é $\mu^{+}$-resolúvel.

No teorema 4.6 mostramos que se um espaço topológico é $n$-resolúvel, para todo número natural $n>1$, então o mesmo também é $\omega$-resolúvel. O próximo resultado mostra que não é possível generalizar tal teorema para todo cardinal infinito $\lambda$.

Teorema 6.3.6. Para quaisquer dois cardinais $\kappa$ e $\lambda$, com

$$
\omega<c f(\lambda)=\lambda \leq \kappa
$$


existe um espaço topológico completamente regular que não é $\lambda$-resolúvel e que é, ainda, hereditariamente $\mu$-resolúvel, para todo cardinal $\mu<\lambda$.

Demonstração. Fixemos a seqüência de cardinais

$$
\vec{\lambda}=\left\{\lambda_{\zeta}: \zeta<\lambda\right\}
$$

de modo que $\lambda_{\zeta}=\rho$, para todo $\zeta<\lambda$, se $\lambda=\rho^{+}$for um cardinal sucessor e de modo que $\lambda_{\zeta}=\omega_{\zeta}$, para todo $\zeta<\lambda$, se $\lambda$ for um cardinal limite.

De acordo com o teorema 6.2 .5 , existem $\mathbb{B}$ e $\mathbb{D}$ duas famílias de partições de $\kappa$,

$$
\mathbb{B}=\left\{\left\langle B_{\xi}^{0}, B_{\xi}^{1}\right\rangle: \xi<2^{\kappa}\right\}
$$

e

$$
\mathbb{D}=\left\{\left\langle D_{\zeta}^{i}: i<\lambda_{\zeta}\right\rangle: \zeta<\lambda\right\}
$$

tais que $\mathbb{B} \cup \mathbb{D}$ é $\kappa$-independente, isto é,

$$
|\mathbb{B}[\epsilon] \cap \mathbb{D}[\eta]|=\kappa
$$

para toda $\eta \in \mathbb{F} \mathbb{N}(\vec{\lambda})$ e toda $\epsilon \in \operatorname{Fn}\left(2^{\kappa}, 2\right)$. Portanto,

$$
\mathcal{D}=\{\mathbb{D}[\eta]: \eta \in \mathbb{F} \mathbb{N}(\vec{\lambda})\}
$$

é uma família de subconjuntos $\kappa$-densos do espaço topológico $X_{\mathbb{B}}$.

Podemos, então, aplicar o teorema 6.2 .6 para $\mathbb{B}$ e $\mathcal{D}$, obtendo assim uma família $\mathbb{C}$ de partições de $\kappa$ satisfazendo as propriedades (1) a (4) do teorema em questão. Mostraremos que o espaço topológico $X_{\mathbb{C}}$ não é $\lambda$-resolúvel, mas é hereditariamente $\mu$-resolúvel, para todo $\mu<\lambda$.

Afirmação. Para cada $\mathcal{E} \in[\mathcal{D}]^{\lambda}$, existe $\mathcal{F} \in[\mathcal{E}]^{\lambda}$ tal que $D \cap D^{\prime} \in \mathcal{D}$ (e, portanto, $D \cap D^{\prime}$ é denso em $\left.X_{\mathbb{C}}\right)$, quando $\left\{D, D^{\prime}\right\} \in[\mathcal{F}]^{2}$. 
Demonstração. Podemos escrever

$$
\mathcal{E}=\left\{\mathbb{D}\left[\eta_{\gamma}\right]: \gamma<\lambda\right\}
$$

onde $\eta_{\gamma} \in \mathbb{F} \mathbb{N}(\vec{\lambda})$, para todo $\gamma<\lambda$. Como $\lambda=\operatorname{cf}(\lambda)>\omega$, existe $K \in[\lambda]^{\lambda}$ tal que

$$
\left\{\operatorname{dom} \eta_{\gamma}: \gamma \in K\right\}
$$

forma um $\Delta$-sistema de raiz $K^{*}$. Assim,

$$
\prod_{i \in K^{*}} \lambda_{i}<\lambda
$$

Como $\lambda$ é regular, existem $I \in[K]^{\lambda}$ e $\eta \in \prod_{i \in K^{*}} \lambda_{i} \subset \mathbb{F} \mathbb{N}(\vec{\lambda})$ tal que

$$
\eta_{\gamma}\left\lceil_{K^{*}}=\eta\right.
$$

para cada $\gamma \in I$. Tomando

$$
\mathcal{F}=\left\{\mathbb{D}\left[\eta_{\gamma}\right]: \gamma \in I\right\}
$$

temos que $\eta_{\gamma} \cup \eta_{\delta} \in \mathbb{F} \mathbb{N}(\vec{\lambda})$, para qualquer $\{\gamma, \delta\} \in[I]^{2}$. Conseqüentemente,

$$
\mathbb{D}\left[\eta_{\gamma}\right] \cap \mathbb{D}\left[\eta_{\delta}\right]=\mathbb{D}\left[\eta_{\gamma} \cup \eta_{\delta}\right] \in \mathcal{D}
$$

Em virtude da afirmação acima e do fato de $X_{\mathbb{C}}$ ser CCC, decorre da proposição 6.1.11 que $X_{\mathbb{C}}$ não é $\lambda$-resolúvel.

Fixemos $\mu<\lambda$ um cardinal. Mostremos que todo $\mathbb{D}[\eta] \in \mathcal{D}$ é $\mu$-resolúvel. Para tanto, seja $\zeta \in \lambda \backslash$ dom $\eta$, com $\lambda_{\zeta} \geq \mu$. Consideremos, para cada $\gamma<\lambda_{\zeta}$, a função $\eta_{\gamma}^{*} \in \mathbb{F} \mathbb{N}(\vec{\lambda})$ tal que $\operatorname{dom} \eta_{\gamma}^{*}=\operatorname{dom} \eta \cup\{\zeta\}, \eta_{\gamma}^{*} \Gamma_{\operatorname{dom} \eta}=\eta$ e $\eta_{\gamma}^{*}(\zeta)=\gamma$. A família

$$
\left\{\mathbb{D}\left[\eta_{\gamma}^{*}\right]: \gamma<\lambda_{\zeta}\right\}
$$


constitui uma partição de $\mathbb{D}[\eta]$ em $\lambda_{\zeta} \geq \mu$ subconjuntos densos. Como $X_{\mathbb{C}}$ é NODEC e $\mathcal{D}$-forçado, da proposição 6.1 .14 segue que $X_{\mathbb{C}}$ é hereditariamente $\mu$-resolúvel.

Destacamos, ainda, que como $\Delta\left(X_{\mathbb{C}}\right)=\kappa, X_{\mathbb{C}}$ é um espaço topológico denso em si mesmo e, portanto, é $\mu$-resolúvel, para todo $\mu<\lambda$.

Bhaskara Rao demonstrou, em [3], que se $\lambda$ é um cardinal singular $\operatorname{com} \operatorname{cf}(\lambda)=\omega$ e se $X$ é um espaço topológico que é $\mu$-resolúvel, para todo $\mu<\lambda$, então $X$ é $\lambda$-resolúvel. Contudo, a questão abaixo permanece sem solução.

Problema 6.3.7. Assuma que $\lambda$ é um cardinal singular com $c f(\lambda)>\omega$ e que $X$ é um espaço topológico $\mu$-resolúvel para todo $\mu<\lambda$. É verdade que $X$ é $\lambda$-resolúvel? 


\section{Referências Bibliográficas}

[1] O. T. Alas, L. R. Junqueira, M. D. Passos, A. H. Tomita, Topologia Geral, Notas de aula (1997).

[2] O. T. Alas, M. Sanchis, M. G. Tkachenko, V. V. Tkachuk, R. G. Wilson, Irresolvable and submaximal spaces: homogeneity versus $\sigma$-discreteness and new ZFC examples, Topology Appl. 107 (2000) 259-273.

[3] K. P. S. Bhaskara Rao, On §-resolvability, preprint.

[4] W. W. Comfort, S. García-Ferreira, Resolvability: a selective survey and some new results, Topology Appl. 74 (1996) 149-167.

[5] E. K. van Douwen, Applications of maximal topologies, Topology Appl. 51 (1993) 125139.

[6] F. W. Eckertson, Resolvable, not maximally resolvable spaces, Topology Appl. 79 (1997) $1-11$.

[7] R. Engelking, General Topology, Heldermann Verlag (1989).

[8] E. Hewitt, A problem of set-theoretic topology, Duke Math. J. 10 (1943) 309-333.

[9] A. Illanes, Finite and w-resolvability, Proc. Amer. Math. Soc. 124 (1996) 1243-1246. 
[10] T. Jech, Set Theory, Springer Monographs in Mathematics (2003).

[11] I. Juhász, Cardinal functions in Topology - ten years later, Mathematical Centre Tracts 123, Mathematisch Centrum (1980).

[12] I. Juhász, L. Soukup, Z. Szentmiklóssy, D-forced spaces: a new approach to resolvability, Topology Appl. 153 (2006) 1800-1824.

[13] K. Kunen, Set Theory - An Introduction to Independence Proofs, North Holland Publishing Company (1980).

[14] D. Rose, K. Sizemore, B. Thurston, Strongly irresolvable spaces, International Journal of Mathematics and Mathematical Science (2006) 1-12.

[15] L. A. Steen, J. A. Seebach, Jr., Counterexamples in Topology, Springer-Verlag (1986). 


\section{Índice Remissivo}

$\Delta$-sistema, 8

$\kappa$-denso, 10

$\mathcal{D}$-mosaico, 102

$\mathcal{D}$-mosaico parcial, 102

$\mathcal{D}$-pedaço, 102

$\pi$-network, 32

aberto

regular, 12

aberto-fechado, 13

caráter, 10

caráter de dispersão, 24

celularidade, 11

densidade, 10

espaço topológico, 9

$T_{0}, 11$

$T_{1}, 11$

$T_{2}, 11$

$T_{3}, 12$

$T_{4}, 12$
$T_{2 \frac{1}{2}}, 12$

$T_{3 \frac{1}{2}}, 12$

$\kappa$-maximal, 62

com respeito à propriedade $\mathcal{P}, 64$

$\kappa$-resolúvel, 29

$\mathcal{D}$-forçado, 103

CCC, 124

completamente regular, 12

de Hausdorff, 11

de Tychonoff, 12

de Urysohn, 12

denso em si mesmo, 10

discreto, 10

extremamente desconexo, 97

hereditariamente $\kappa$-resolúvel, 110

HI, 43

irresolúvel, 16

localmente compacto, 14

maximal, 45

maximalmente resolúvel, 32 
NODEC, 81

normal, 12

OHI, 42

regular, 12

resolúvel, 16

semi-regular, 12

submaximal, 44

zero-dimensional, 13

expansão, 54

família

celular, 11

de partições

$\kappa$-independente, 112

independente, 113

separante, 113

independente, 9

independente maximal, 9

filtro, 5

cofinito, 5

funções

cardinais, 10

parciais finitas, 5

lema

do $\Delta$-sistema, 9

do refinamento disjunto, 34 peso, 10

primeiro axioma de enumerabilidade, 10

propriedade

da intersecção finita, 6

de expansão monotônica, 64

invariante por expansões, 62

raiz

do $\Delta$-sistema, 8

segundo axioma de enumerabilidade, 10

subconjunto

abundante, 81

raro, 81

subespaço

$\kappa$-resolúvel, 29

resolúvel, 20

suporte, 9

teorema

da decomposição de Hewitt, 43

de Hewitt-Marczewski-Pondiczery, 13

topologia, 9

cofinita, 10

induzida, 13

ultrafiltro, 6

livre, 7 